\title{
Wing transparency in butterflies and moths: structural diversity, optical properties, and ecological relevance
}

\author{
Doris Gomez (D),${ }^{1,8}$ Charline Pinna, ${ }^{2}$ Jonathan Pairraire, ${ }^{3}$ Mónica Arias, ${ }^{1,2}$ Jérôme Barbut, ${ }^{2}$ \\ Aaron Pomerantz, ${ }^{4,5}$ Willy Daney de Marcillac, ${ }^{3}$ Serge Berthier, ${ }^{3}$ Nipam Patel,, 4 \\ Christine Andraud, ${ }^{7}$ and Marianne Elias ${ }^{2}$ \\ ${ }^{1} C E F E$, University of Montpellier, CNRS, EPHE, IRD, Montpellier, France \\ ${ }^{2} I S Y E B, U M R$ 7205, CNRS, MNHN, EPHE, Sorbonne University, Paris, France \\ ${ }^{3}$ INSP, CNRS, Sorbonne University, Paris, France \\ ${ }^{4}$ Marine Biological Laboratory, Woods Hole, Massachusetts 02543 USA \\ ${ }^{5}$ Department Integrative Biology, University of California Berkeley, Berkeley, California 94720 USA \\ ${ }^{6}$ University of Chicago, Chicago, Illinois 60607 USA \\ ${ }^{7}$ CRC, MNHN, Paris, France
}

Citation: Gomez, D., C. Pinna, J. Pairraire, M. Arias, J. Barbut, A. Pomerantz, W. Daney de Marcillac, S. Berthier, N. Patel, C. Andraud, and M. Elias. 2021. Wing transparency in butterflies and moths: structural diversity, optical properties, and ecological relevance. Ecological Monographs 00(00):e01475. 10.1002/ecm. 1475

Abstract. In water, transparency seems an ideal concealment strategy, as testified by the variety of transparent aquatic organisms. By contrast, transparency is nearly absent on land, with the exception of insect wings, and knowledge is scarce about its functions and evolution, with fragmentary studies and no comparative perspective. Lepidoptera (butterflies and moths) represent an outstanding group to investigate transparency on land, as species typically harbor opaque wings covered with colored scales, a key multifunctional innovation. Yet, many Lepidoptera species have evolved partially or fully transparent wings. At the interface between physics and biology, the present study investigates wing transparency in 123 Lepidoptera species (from 31 families) for its structural basis, optical properties, and biological relevance in relation to visual detection (concealment), thermoregulation, and protection against UV. Our results suggest that transparency has likely evolved multiple times independently. Efficiency at transmitting light is largely determined by clearwing microstructure (scale shape, insertion, coloration, dimensions, and density) and macrostructure (clearwing area, species size, or wing area). Microstructural traits, scale density and dimensions, are tightly linked in their evolution, with different constraints according to scale shape, insertion, and coloration. Transparency appears highly relevant for concealment, with size-dependent variations. Links between transparency and latitude are consistent with an ecological relevance of transparency in thermoregulation, and not so for protection against UV radiation. Altogether, our results shed new light on the physical and ecological processes driving the evolution of transparency on land and underline that transparency is a more complex coloration strategy than previously thought.

Key words: Lepidoptera; microstructure; structural strategy; thermoregulation; transparency; UV protection; vision.

\section{INTRODUCTION}

Following the invisibility myth, transparency seems an ideal camouflage strategy: being "hidden in plain sight" works whatever the background, from all viewpoints and irrespective of behavior (Cuthill 2019). The large "success story" of transparency in water as a protection against predators (especially in pelagic habitats where there is nowhere to hide) is attested by its broad phylogenetic distribution since transparency spans seven phyla

Manuscript received 26 March 2021; revised 9 April 2021; accepted 16 April 2021.

Corresponding Editor: Matthew L. Forister.

${ }^{8}$ E-mail: doris.gomez@cefe.cnrs.fr including Arthropoda, Mollusca, Annelida, Chordata, and Cnidaria (Johnsen 2001). By contrast, transparency is nearly absent on land, and it is almost entirely confined to insect wings (one phylum). This contrast can be explained by physical factors: compared to water, larger refractive index mismatch between air and biological tissues produces higher light reflection that ruins invisibility (Johnsen 2001). In addition, greater ultraviolet (UV) radiation on land imposes greater selection on UV protection often through light absorption by pigments.

Research in transparent aquatic organisms has shown a role for concealment from visually hunting predators, and predators' ability to break this camouflage (e.g., Tuthill and Johnsen 2006). As underlined by Johnsen (2014), many questions are left unanswered about 
transparency: the structural bases of transparency (Bagge 2019), its genetic and developmental pathways, its functional roles in vital functions like thermoregulation and potential trade-offs with optics, and the selective pressures driving its evolution and its design. Comparative studies at a broad interspecific level are absent, yet they are essential to better understand the links between structural diversity and adaptive functions of transparency. The lack of studies is even more crucial for transparency on land, where knowledge is scarce, with fragmentary monographic studies by physicists using bioinspired approaches, based on transparent wing antireflective, hydrophobic and antifouling properties (e.g., Deparis et al. 2014, Liu et al. 2016, Elbourne et al. 2017).

Within insects, Lepidoptera represent an outstanding group to explore these questions. While most insects harbor transparent wings, Lepidoptera are characterized by wings covered with scales. Scales are chitin extensions that are usually long and large and that often contain pigments or structures that interact with light, thereby producing opaque color patterns (e.g., Stavenga et al. 2014). Wings covered by scales represent an evolutionary innovation involved in many functions such as antipredator defenses (camouflage, deflection, aposematism, e.g., Stevens et al. 2008), communication (Kemp 2007), thermoregulation (Miaoulis and Heilman 1998, Berthier 2005, Krishna et al. 2020), and water repellency (Wagner et al. 1996, Wanasekara and Chalivendra 2011). In this opaque world, many species from different families have evolved partially or totally transparent wings. The handful of relevant biophysical studies in Lepidoptera suggests that an important aspect of structural diversity-having a nude membrane (completely lacking scales) or with scales of various morphologies, insertion angle on the wing membrane, and colorationmay underlie the occurrence of transparency in many Lepidoptera lineages (Yoshida et al. 1997, HernandezChavarria et al. 2004, Berthier 2007, Goodwyn et al. 2009, Wanasekara and Chalivendra 2011, Stavenga et al. 2012, Siddique et al. 2015). This opens questions regarding the phylogenetic extent of transparency, the diversity and evolution of optical properties and of the underlying structures, the existence, if any, of structural constraints on transparency, and the ecological relevance of transparency on land.

Using a large data set comprising 123 clearwing Lepidoptera species encompassing 31 families, we examine the phylogenetic distribution of transparency in Lepidoptera. Theoretical and empirical studies on aquatic organisms suggest there exist several structural and optical routes to transparency (Johnsen 2001), highlighting the interest of quantifying structural diversity in terrestrial organisms.

First, we aim to quantify the extent of structural diversity for transparency in Lepidoptera at various scales: macrostructure (wing size, wing area, clearwing area, and proportion of clearwing area) and microstructure (presence of scales, type, insertion, and coloration). We assess to what extent structural features are conserved across the phylogeny, and whether some of the features are involved in correlated evolution, which can help us reveal evolutionary constraints. We quantify the investment in transparency, relative to what is invested in the opaque scales.

Second, we aim to examine the potential impact of macro- and microstructure on optical properties. We assess to what extent scale type, insertion, coloration, and density influence optical transparency, and we hypothesize that lower wing membrane coverage by scales (smaller surface and/or in lower density) should result in higher levels of optical transparency.

Third, we test the ecological relevance of transparency for visual detection, and especially for concealment. The prominent role of transparency in camouflage shown so far (Johnsen 2014, Arias et al. 2019, 2020, McClure et al. 2019) suggests that visually hunting predators may exert an important selective pressure on the evolution of transparency on land as they can prey on flying or resting diurnal prey as well as on nocturnal resting prey. We hypothesize that higher levels of optical transparency should yield lower visual contrasts with the background as perceived by natural predators. Given the principle of transparency (giving visual access to what is behind, under any light), this should apply to both visual systems found in birds and in various visual conditions of ambient light and background.

Fourth, we test the ecological relevance of transparency for thermoregulation. The thermal melanism hypothesis states that individuals from colder places (at higher latitudes or altitudes) gain thermal benefits from being more strongly pigmented as radiation absorption helps heating (Bogert 1949). This hypothesis has received support from comparative analyses at large taxonomical and geographical scales (e.g., Zeuss et al. 2014, Xing et al. 2018, Stelbrink et al. 2019) and from analyses at a species level in Lepidoptera (e.g., Colias species in Ellers and Boggs [2004]) showing that thermal benefits come from melanization of the proximal wing, close to the body. Recent large-scale comparative analyses in opaque butterflies have shown that body and proximal wing coloration correlates to climate in the nearinfrared (700-1,100 nm) range but not so below $700 \mathrm{~nm}$ where not only thermoregulation but also vision operates (Munro et al. 2019). Hence, wavelength absorption should be higher at high latitudes compared to low latitudes, especially in the infrared range where most of the thermal radiative exchanges take place.

Fifth, we finally test the ecological relevance of transparency for UV protection. Exposure to highly energetic and penetrating UVB (280-315 nm) and UVA (315$400 \mathrm{~nm}$ ) radiations has detrimental effects on physiology, fecundity, and survival in terrestrial living organisms (e.g., in insects Zhang et al. 2011). The absorption of UV radiation by pigments is efficient to prevent UV harmful effects, as shown in transparent fish larvae, 
where exposure to UV light rapidly induces melanization as a UV protection (Mueller and Neuhauss 2014). Levels of UV radiation are higher at low latitudes than at higher latitudes (Beckmann et al. 2014). Hence, if UV protection is a strong selective force, absorption of UV radiation is expected to be more important at low latitudes. Moreover, pathogens are more important in warmer and more humid regions, hence in lower latitudes. Stronger melanization at lower latitudes has been shown to provide protection against pathogens (True 2003). Hence, a higher UV absorption at lower latitudes would also potentially protect against pathogens.

Testing the potential role of transparency in thermoregulation or UV protection generates two mutually exclusive hypotheses. In optics, the light received by an object can be either transmitted, reflected, or absorbed. Variation in light transmission can indicate variation in absorption if reflection levels are maintained at similar levels. We hypothesize that species with fully or partially transparent wings living at increasing distance from the equator should transmit less light (absorb more) through their wings (at least in the near-infrared range) if transparency plays a role in thermoregulation, as light absorption by pigments is a way of gaining energy. Conversely, if transparency plays a role in protection against UV, species with fully or partially transparent wings living at increasing distance from the equator are expected to absorb less in the UV range, and thus to transmit more. The present study thus addresses for the first time the links between structure and optics at a broad phylogenetic scale to understand the "small success story of transparency on land," its evolution, and putative functions in relation to camouflage and thermoregulation.

\section{Methods}

\section{Specimens and ecological data}

We looked for clearwing species in the Lepidoptera collection of the French Museum of Natural History, by sampling as many families as possible to increase the power of comparative analyses. We found clearwing species in 31 out of the 124 existing Lepidoptera families (Supplementary file File1_SpeciesList.txt, see families in Appendix S1: Fig. S1) and gathered a total of 123 species. We included transparent and translucent species (i.e., when a text can be read when placed just behind but not from a distance, like a tracing paper) in this data set as they were likely on a continuum of light transmission and it was important to see whether this continuum entailed qualitative or quantitative changes. We took one specimen per species (see Fig. 1 for some examples; sex information was unavailable for most specimens and thus discarded). Those specimens were often unique or precious, which prevented us from conducting destructive measurements. There were 77 specimens for which labels specified exact collect location that could be tracked down to GPS coordinates (see Appendix S1: Fig. S2).

\section{Structure measurements}

Museum specimens were photographed using a D800E Nikon camera (Nikon) equipped with a $60 \mathrm{~mm}$ lens, placed on a stand with an annular light. Photos were then analysed using ImageJ (Schneider et al. 2012) to extract descriptors of wing macrostructure: wing length (expressed in mm, see Appendix S1: Fig. S2 for its phylogenetic distribution in the forewing), wing area $\left(\mathrm{mm}^{2}\right)$, and clearwing area (the surface of transparent area in $\mathrm{mm}^{2}$ ), for the forewing and hindwing separately. We defined and computed proportion of clearwing area as the ratio clearwing area/wing area, i.e., the proportion of the total wing area occupied by transparency (see Appendix S1: Fig. S2 for its phylogenetic distribution in the forewing).

Museum specimens were also photographed with microscopes (Zeiss Stereo Discovery V20 [Zeiss] and a high-resolution Keyence VHX-5000 [Keyence]) to get detailed images of the dorsal side of transparent and opaque zones of each wing. These images were analysed using the built-in measurement software to describe wing microstructure. For each wing separately, we measured scale density (number of scales per $\mathrm{mm}^{2}$, as zero for nude membrane, whatever the density of scale sockets, see Appendix S1: Fig. S2 for its phylogenetic distribution in the forewing), length $(\mu \mathrm{m})$, and width $(\mu \mathrm{m})$. We computed scale surface (in $\mu \mathrm{m}^{2}$ ) as the product of length by width, and scale coverage as the product of scale surface (expressed in $\mathrm{mm}^{2}$ ) by scale density.

As shown from examples in Fig. 1, the transparent zone showed diversity in scale morphological type, in scale insertion, and in scale coloration. (1) In scale morphological type (hereafter referred to as type): no scale $(\mathrm{N})$, bifid or monofid "hair-like" piliform scales (P), scales with any shape other than piliform, hereafter called lamellar scales (L) as in Kristensen et al. (2003), presence of both lamellar scales and piliform scales (PL). (2) In scale insertion on the membrane: erect (E) or flat (F) scale, or unknown insertion (U, when scales were absent). (3) In scale coloration: colored (C) or transparent ( $T$, when scales were partly or totally transparent), or unknown coloration (U, when scales were absent).

We define as structural strategy the combination of the following traits: scale type, insertion, and coloration (examples in Fig. 1). The name of a structural strategy is thus composed of the abbreviated letters for scale type, insertion, and coloration, in this order. The NUU strategy (hereafter called N strategy; e.g., Senecauxia coraliae or Chalioides ferevitrea) has no scales, unknown insertion, and unknown coloration. PEC (e.g., Pseudohaetera hypaesia) is based on colored erect piliform scales. PFC (e.g., Attacus atlas) is based on colored flat piliform scales. LEC (e.g., Isostola flavicollaris) is based on erect colored lamellar scales while LET (e.g., Oxynetra semihyalina) is based on erect transparent lamellar scales. LFC (e.g., Cressida 

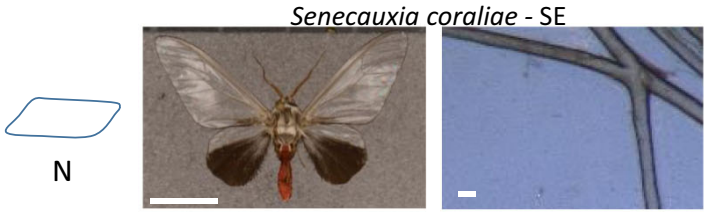

Chalioides ferevitrea - $\mathrm{CH}$

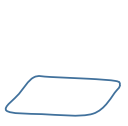

N

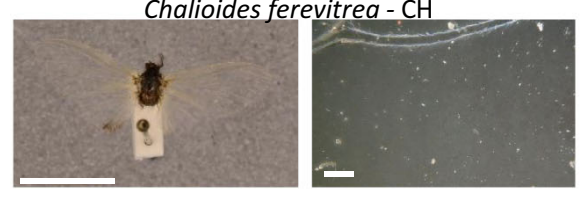

Pseudohaetera hypaesia - PS

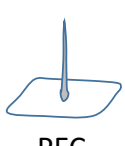

PEC
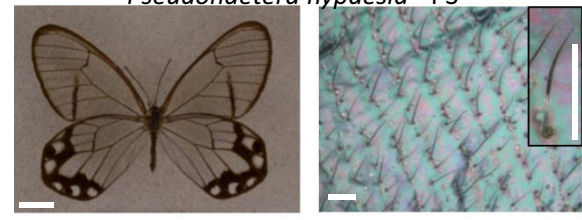

Attacus atlas - AT

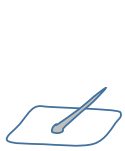

PFC
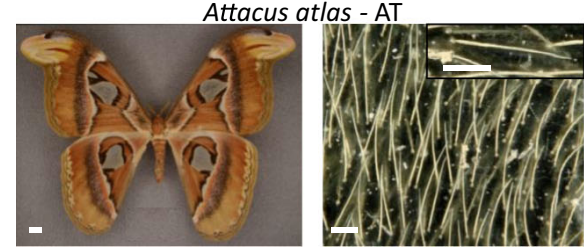
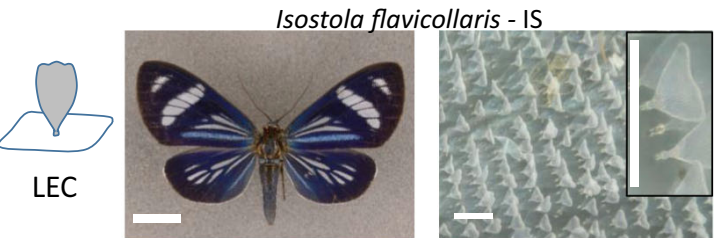

Oxynetra semihyalina - OX

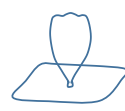

LET
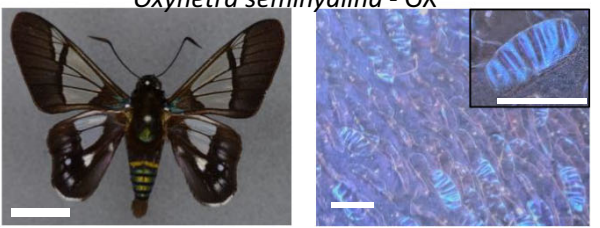

Cressida cressida - CR
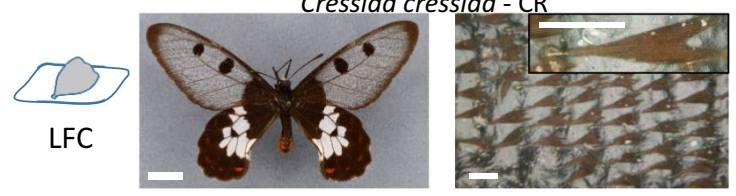

Diaphania unionalis - DI
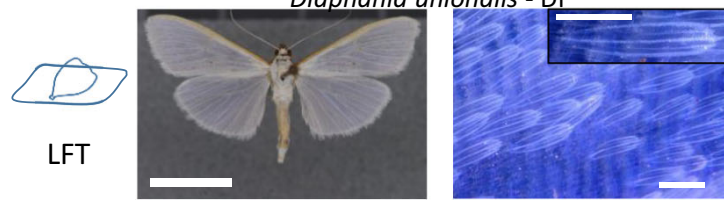

FIG. 1. Examples of structural strategies in Lepidoptera. A structural strategy is defined as the combination of scale type, insertion, and coloration. Type is $\mathrm{P}$, piliform scales, or L, lamellar scales (the combination of piliform and lamellar scales was assimilated to lamellar scales); insertion is $\mathrm{E}$, erect, or $\mathrm{F}$, flat; and coloration is $\mathrm{C}$, colored, or $\mathrm{T}$, transparent. The $\mathrm{N}$ strategy has no scales, no insertion, and no coloration. Bar scales are $1 \mathrm{~cm}$ for entire specimens (left columns), $100 \mu \mathrm{m}$ for microscopic imaging (right columns and scale details). Species are the erebid Senecauxia coraliae (SE), the psychid Chalioides ferevitrea $(\mathrm{CH})$, the nymphalid Pseudohaetera hypaesia (PS), the saturniid Attacus atlas (AT), the erebid Isostola flavicollaris (IS), the hesperid Oxynetra semihyalina (OX), the papilionid Cressida cressida (CR), and the crambid Diaphania unionalis (DI). Notice that we chose not to illustrate the PL category (combination of both scales and piliform scales) because, due to their rarity in the data set, they were pooled with the L category in subsequent analyses. Symbols for lamellar and piliform are meant as illustrative and do not represent exact scale morphologies encountered in the documented species.

cressida) is based on lamellar scales flat on the wing membrane and colored, while LFT (e.g., Diaphania unionalis) is based on lamellar scales flat on the wing membrane and transparent. The structural strategies based on a combination of lamellar scales and piliform scales (starting with PL), are not illustrated because of their rarity. PL strategies were pooled with strategies based on lamellar scales (starting with L) of their corresponding insertion and coloration, for several reasons: (1) the exploration of structural changes in scale length and width could only address one type of scale, not two, (2) strategies involving the combination of lamellar scales and piliform scales were rare and their effects could not be easily investigated with statistical models. (3) In PLF strategies (PLFC and PLFT), both lamellar scales and piliform scales were in similar density and in PLE strategies (PLEC and PLET), lamellar scales were in greater density than piliform scales, which suggested that lamellar scales played a similar role or a greater role than piliform scales for aspects linked to scale density. Although defined for the transparent zone, this terminology also applied to the opaque zone, but fewer strategies existed (Appendix S1: Table S1).

\section{Optical measurements and visual perception modeling}

We measured direct (specular) transmittance from 300 to $1,100 \mathrm{~nm}$, using a deuterium-halogen lamp (Avalight DHS, Avantes), separate lighting and collecting optic fibers (FC-UV200-2-1.5 × 100, Avantes), and a spectrometer (Starline Avaspec-2048 L, Avantes). Fibers were aligned $5 \mathrm{~mm}$ apart and the wing sample was placed perpendicular between them at equal distance, guaranteeing that we measured direct (specular) transmittance and not diffuse transmittance. The incident light beam made a $1 \mathrm{~mm}$ diameter spot. Spectra were taken relative to a dark (light off) and to a white reference (no sample between the fibers) measurement. For each species and wing, we took five measurements in the transparent zone. We did not measure diffuse transmittance as it required using an integrating sphere and put specimens in contact with it, which would have damaged the specimens.

We analyzed spectral shape using Avicol v6 (available online) $^{9}$ and the pavo R package (Maia et al. 2019) to

\footnotetext{
${ }^{9}$ http://sites.google.com/site/avicolprogram/
} 
extract physically and biologically relevant parameters. We computed the mean transmittance over (300$700 \mathrm{~nm}$ ), which described the level of transparency. In addition, we computed the proportion of UV transmittance as the ratio (total transmittance over (300$400 \mathrm{~nm}$ )/total transmittance over $(300-700 \mathrm{~nm})$ ), i.e., the proportion of the total amount of transmitted light that occurred in the ultraviolet range. Notice that perfect optical transparency consists in transmitting $100 \%$ of the incident light at all wavelengths. It yields a proportion of UV transmittance of 0.25 . Chitin, of which wings and wing scales are made, absorbs negligibly above $500 \mathrm{~nm}$, but more as wavelength decreases, especially in the ultraviolet range (Azofeifa et al. 2012, Stavenga et al. 2014). Given that absorption + reflection + transmission $=1$, a loss in transmission indicates an increase in absorption if reflection is maintained at similar levels. We also computed the mean transmittance separately for the ultraviolet range $(300-400 \mathrm{~nm})$, the humanvisible range $(400-700 \mathrm{~nm})$ and the near infrared range $(700-1,100 \mathrm{~nm})$. We could thus disentangle the nearinfrared range where only thermoregulation can act as a selective pressure from shorter wavelengths where vision also acts on coloration (as in Munro et al. 2019), and we could isolate the UV range to test the potential role of transparency as a parasol against UV radiation.

We also aimed to test whether the physical transparency, mean transmittance or proportional UV transmittance, translated into a biologically meaningful transparency, namely a visual contrast with a background as perceived by a potential bird predator. Exploring a large variety of visual configurations ensured robust conclusions. We thus analysed spectra using Vorobyev and Osorio's discriminability model (Vorobyev and Osorio 1998), which quantifies color and brightness contrasts between (1) an object and a background, seen (2) under an illuminant and (3) by a visual system. (1) We considered three visual conditions (see details in Appendix S1: Fig. S3): a clearwing patch could be seen against a sky, a leaf, or a trunk background (see McClure et al. (2019) and Arias et al. (2020) for average leaf and trunk spectra respectively). (2) We considered three natural illuminants that covered most of diurnal conditions encountered in terrestrial environments, i.e., in forests and open areas, as defined by Endler (1993): woodland shade (found in forest canopy and forest edge), forest shade (found in forest understorey), and large gap (found in open areas and in forest canopy). (3) We modeled both vision systems found in birds, the UVS vision and the VS vision, as both types are found in insectivorous birds (Odeen et al. 2011). For the UVS vision system, we used the spectral data of the Blue Tit (Cyanistes caeruleus) and its relative cone densities of 1:1.9:2.7:2.7 for UVS:S:M:L (Hart et al. 2000). For the VS vision system, we used the spectral data for the Shearwater (Puffinus pacificus) and its relative cone densities of 1:0.7:1:1.4 for VS:S:M:L (Hart 2004). For both models, we assumed quantum noise, a
Weber fraction of 0.1 for chromatic vision (Maier and Bowmaker 1993, Lind et al. 2014) and 0.2 for achromatic vision (average of the two species studied in Lind et al. 2013). We treated brightness detection as performed by double cones (Campenhausen and Kirshfeld 1998). We used the Blue Tit and the Shearwater as their sensitivity peaks are similar to most species of their groups, making them good representative of their respective UVS and VS vision type. Moreover, they are among the very few species for which we know other parameters needed to accurately model bird visual perception, such as relative cone densities, oil droplet transmission spectra and ocular media transmission spectra (Hart et al. 2000, Hart 2004, Hart and Vorobyev 2005).

\section{Phylogeny reconstruction}

We built the phylogeny for the 123 clearwing species representing the 31 families included in our data set, as follows. First, for each of the 123 clearwing species in our data set, we searched for DNA sequences in GenBank and BOLD (Ratnasingham and Hebert 2007), and if none was available, we took a species from the same genus, tribe, subfamily, or family as a substitute, choosing the lowest taxonomic level for which we had a substitute (Supplementary file File1_SpeciesList.txt for the list of analysed species and substitute species). Second, we incorporated 60 additional species from families where we only had a few species (e.g., Bombycidae, Cossidae, Drepanidae, Limacodidae, Megalopygidae, Noctuidae, Notodontidae, and Pyralidae, among others), in order to infer tree topology with more certainty. These species were chosen of the same subfamily or, if not possible, in the same family to get three to five species per family. We thus obtained a set of 183 species to build the tree. We used DNA sequences for the mitochondrial COI and COII genes, and for the nuclear CAD, EF1, GADPH, IDH, MDH, RpS5, and wg genes (Supplementary file File2_SpeciesSequences.txt). We aligned the sequences with CodonCodeAligner (version 4.2.7, CodonCode Corporation, available online $)^{10}$ and concatenated them with PhyUtility (version 2.2, Smith and Dunn 2008). The data set was then partitioned by gene and codon positions and the best models of substitution were selected over all models implemented in BEAST 1.8.3 (Drummond and Rambaut 2007), using the "greedy" algorithm and linked rates implemented in Partition Finder 2.1.1 (Lanfear et al. 2017, Supplementary file File5_PartitionFinder_BestScheme.txt). We constrained the topology of all families to follow Figure S12.tre in Supplementary Archive 8 from Kawahara et al. (2019), and we used the following secondary calibrations from that figure: node joining Bombycoidea and Lasiocampoidea at $84.05 \mathrm{Myr}$ with $95 \%$ credibility interval $[74.15,94.4])$, Noctuidea ancestor at 77.6 Myr [66.97, 88.57]), node joining Gelechioidea to Bombycoidea at

\footnotetext{
${ }^{10} \mathrm{http}: / /$ www.codoncode.com/
} 
105.23 Myr [93.77, 117.3]), Papilionoidea ancestor at 98.34 Myr [86.85, 110.33]); node joining Sesioidea to Cossoidea at 105.66 Myr [93.4, 118.32]), and tree root at 145.03 Myr [128.96, 161.64]) on Cipres Science Gateway (Miller et al. 2010). We constrained monophyly at genus, tribe, subfamily, and family level. Specifically, species belonging to the same genus (or tribe, subfamily, and family) were considered to form a monophyletic clade. Four independent analyses were run for 10 million generations, with one Markov Chain Monte Carlo each and a sampling frequency of one out of 10,000 generations. We examined the trace of each run, and defined a burnin period for each run independently, using Tracer 1.6 (available online). ${ }^{11}$ We retained the 2 runs that had a stable trace and combined the trees using LogCombiner 1.8.4 (available online). ${ }^{12}$ We then computed the maximum clade credibility (MCC) tree with median node ages using TreeAnnotator 1.8.4 (Drummond et al. 2012). Additional species were then pruned from the tree and we used the resulting MCC tree with 123 species in subsequent comparative analyses (Supplementary file File6_PhylogeneticTree_123species.tre).

\section{Statistical analyses}

All analyses were conducted using the $\mathrm{R}$ environment (R Development Core Team 2013). Data used for the analyses are available as supplementary files (File3_Data_AllColors.txt and File4_Data_SpeciesMeans.txt).

Repeatability analysis. - We assessed the repeatability of optical and structural parameters, using the rptR package (Stoffel et al. 2017). For transparency measurements, we took the five measurements as repetitions of the same species and wing. For wing length, we measured each wing twice and thus obtained two repetitions of the same wing for each species. Regarding the repeatability of the measurements of scale density, length, and width, we measured a small number of scales of the same type, zone, and wing, and tested whether within-group variability was lower than between-group variability. All measurements were found highly repeatable (see sample sizes and results in Appendix S1: Table S2).

Phylogenetic signal and phylogenetic clustering.-We implemented two complementary approaches to estimate the statistical nonindependence among structural trait values due to species phylogenetic relatedness. First, we estimated the amount of phylogenetic signal in each structural and optical variable. For continuous variables, we used both Pagel's lambda (Pagel 1999) and Blomberg's $K$ (Blomberg et al. 2003) implemented in the phytools R package (Revell 2012). For binary variables, we used Fritz and Purvis' $D$ (Fritz and Purvis 2010) implemented in the caper R package (Orme et al.

\footnotetext{
${ }^{11} \mathrm{http}: / /$ beast.bio.ed.ac.uk/tracer

${ }^{12} \mathrm{http} / / /$ beast.bio.ed.ac.uk
}

2018). Second, we assessed to what extent structural features and structural strategies were phylogenetically conserved, in other words we estimated the degree of phylogenetic clustering for structures. We calculated the mean pairwise phylogenetic distances (MPD) for each categorical structural parameter (Webb 2000), using the picante $\mathrm{R}$ package (Kembel et al. 2010). MPD measures the average phylogenetic distance that separates two species sharing a specific trait state. We computed MPD values for the forewing and the hindwing separately, or for both the forewing and the hindwing (in that case we considered all the species that presented the trait on at least one of the wings, the forewing or the hindwing). For a specific trait, we first computed the observed MPD, and then simulated MPD distribution by randomly shuffling trait values on the phylogeny. We then determined whether the observed value was lower than the $5 \%$ lower quantile of the distribution of simulated MPD values, in which case we concluded that the trait was found in species separated by fewer nodes than expected by chance.

Correlated evolution between structural traits.-To assess whether scale presence, type, insertion, and color evolved in a correlated fashion both within and across transparent and opaque zones, we computed Pagel's discrete model for those binary traits and compared the likelihood values from the dependent and independent models, using the FitPagel function, assuming a general formulation of dependency (ARD, i.e., all transition rates being different and the transition rate on character 1 depending on the state of character 2 and vice versa) from phytools (Revell 2012).

General statistical approach for structural constraints, structure-optics relationships, and ecological relevance.We conducted (1) mixed models using the nlme R package (Pinheiro et al. 2020) and (2) Bayesian phylogenetic mixed models with Markov Chain Monte Carlo analyses using the mulTree $\mathrm{R}$ package (Guillerme and Healy 2019). Both approaches are suited to repeated observations but, unlike the former, the latter controls for phylogenetic relatedness. Using both allowed us to assess the influence, if any, of phylogeny on the observed relationships. (1) In the mixed model approach, we selected the best model based on minimization of the Akaike information criterion corrected for sample size $\left(\mathrm{AIC}_{\mathrm{c}}\right)$ and based on the factors supposed to play a role in determining variation in the dependent variable. (2) In the Bayesian phylogenetic approach, we used the model formulated in the classic approach; uninformative priors were used with an inverse-gamma distribution with shape $=0.001$ and scale $=0.001$ for both random effect and residual variances (Hadfield 2010). We took species as random effects. Models were run using two chains of 500,000 iterations with a burn-in of 10,000 and thinning interval of 300. Fixed effects were considered statistically important and supporting the effect considered when the 
$95 \%$ credible intervals exclude zero, less so when only the $90 \%$ credible intervals exclude zero.

Structural constraints. - We aimed to investigate the variation in the proportion of clearwing area in relation to species macro and microstructural features to explore the macro and micro structural constraints on transparency. For this, we took the proportion of clearwing area as the dependent variable, and scale length, insertion, and coloration, as well as wing length, clearwing area, and wing as factors, with meaningful interactions.

We also quantified the relative investment in scales in the transparent zone as compared to the opaque zone. More specifically, we computed the relative investment in a given microstructural trait (scale density, scale length, scale width, scale surface, or scale coverage) for a specific species and wing as Relative investment $_{\text {Trait }}=$ Trait $_{\text {transparent zone }}-$ Trait $_{\text {opaque zone. Values }}$ departing more from zero indicate greater changes: toward a decrease (negative values, economy of investment in the transparent relative to the opaque zone) or an increase (positive values, increase in investment in the transparent relative to the opaque zone). For each structural trait, we took the two relative investment values per species (one per wing) obtained from image analysis as observations and treated species as a random effect. We took the relative investment in a specific trait as the dependent variable. We included as fixed effects category, insertion and color in the transparent zone, scale surface, and coverage in the opaque zone, as well as wing length, clearwing area, and proportion of clearwing area, and relevant interactions between these factors.

Structure-optics relationships.-We explored which structural parameters influenced optical properties in clearwing Lepidoptera. For this purpose, we took the 10 spectral measurements per species as observations and species and wing within species as random effects. We took the mean transmittance over $(300-700 \mathrm{~nm})$ or the proportion of UV transmittance as the dependent variable. We included as fixed effects scale surface, density, category, insertion and color, wing length, clearwing area, and proportion of clearwing area and relevant interactions between these factors.

Ecological relevance.-We explored the ecological relevance of transparency for visual detection and more specifically for concealment, for thermoregulation, and for protection against UV. We took the 10 spectral measurements per species as observations and species and wing within species as random effects. First, we examined whether optical properties translated into perceptual transparency. We took the physical property (mean transmittance over (300-700 $\mathrm{nm}$ ) or proportion of UV transmittance) as the dependent variable. We included as fixed effects the wing, the color contrast and brightness contrast and relevant interactions between these factors.
We expected that greater optical transparency translated into greater concealment (lower visual contrast) to a predator, whatever its visual ability, the ambient light or visual background considered.

Second, we explored the link between transparency and latitude to test a potential role of transparency in thermoregulation or protection against UV. For thermoregulation, Munro et al. (2019) have shown that body and proximal wing coloration vary with latitude in butterflies (lower reflection, hence higher absorption at high latitudes), more strongly in smaller species, and more strongly in the near-infrared range. We thus took the mean transmittance over specific ranges of wavelengths (UV, human-visible, and near-infrared) as the dependent variable and we included latitude (absolute latitudinal distance to the equator), wing length and their interaction as fixed factors. We expected lower transmittance (higher absorption) at higher latitudes if transparency could play a role in thermoregulation.

Protection against UV radiation can be ensured by UV absorption (if reflection was identical, this means lower transmission). Decreasing transmission could be ensured by limiting the absolute transmittance in the UV or, but with a lower expected efficiency, the transmittance in the UV range relative to that in the other wavelength ranges (hence the proportion of UV transmittance). Hence, we took mean transmittance over $(300-400 \mathrm{~nm})$ or the proportion of UV transmittance as dependent variables and tested them against latitude. We expected higher absolute or relative UV transmittance (lower absorption) at higher latitudes if transparency could play a role in UV protection.

\section{Results}

\section{Diversity of microstructures}

Structural investigation showed a high diversity of structural strategies (gathering scale type, insertion, and coloration), as illustrated by a few examples (Fig. 1 and associated spectra in Fig. 2). Transparency could be achieved by the means of a nude wing membrane $(N)$, of piliform scales (P), of lamellar scales (L), or of piliform scales and lamellar scales in combination (PL). When present, scales could be flat $(\mathrm{F})$ or erect $(\mathrm{E})$ on the wing membrane and colored (C) or transparent (T; Fig. 3). Rather counterintuitively, lamellar scales (L) are by far the most common structural type to achieve transparency (69/123 species, 26/31 families), followed by the absence of scales (N, 33/123 species, 12/31 families) and piliform scales alone ( $\mathrm{P}, 27 / 123$ species and $9 / 31$ families, Appendix S1: Table S1 and Fig. S1). Rarer strategies involved either transparent erect lamellar scales (LET, 9/ 123 species, $6 / 31$ families) or the combination of piliform scales and lamellar scales (PL, 12/123 species, 7/31 families). Within this latter category, the combination of erect piliform scales and lamellar scales was the rarest 


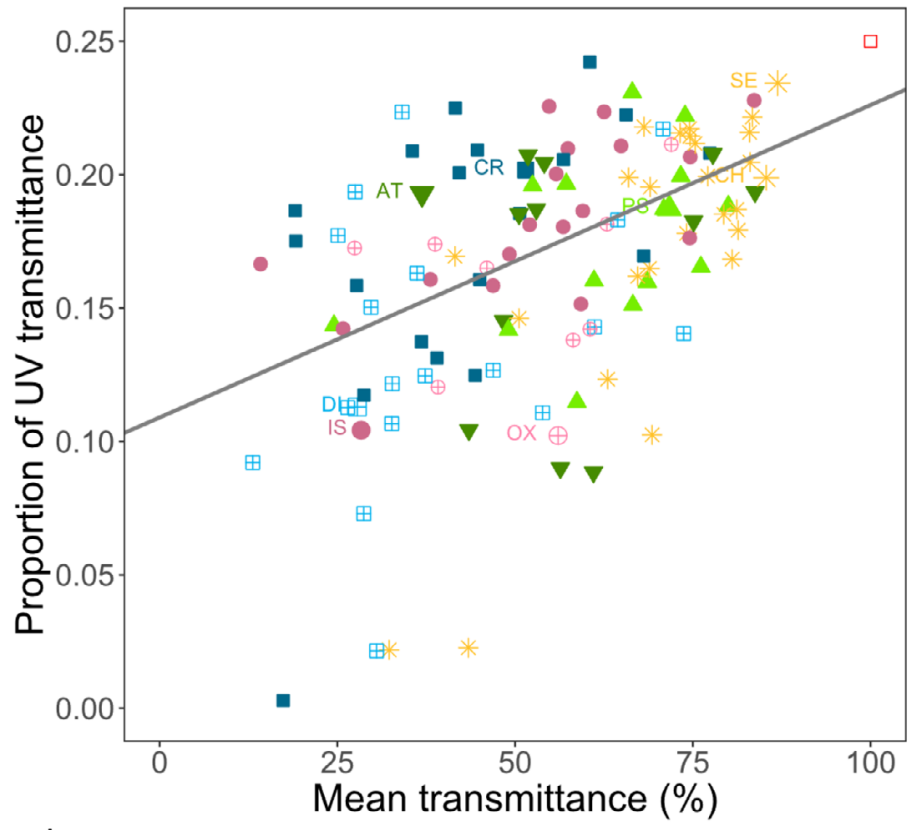

\section{Structural strategy}

b
$\triangle$ PEC
$\checkmark$ PFC
- LEC \& PLEC
$\oplus$ LET \& PLET
- LFC \& PLFC
$\boxplus \quad$ LFT \& PLFT
* N

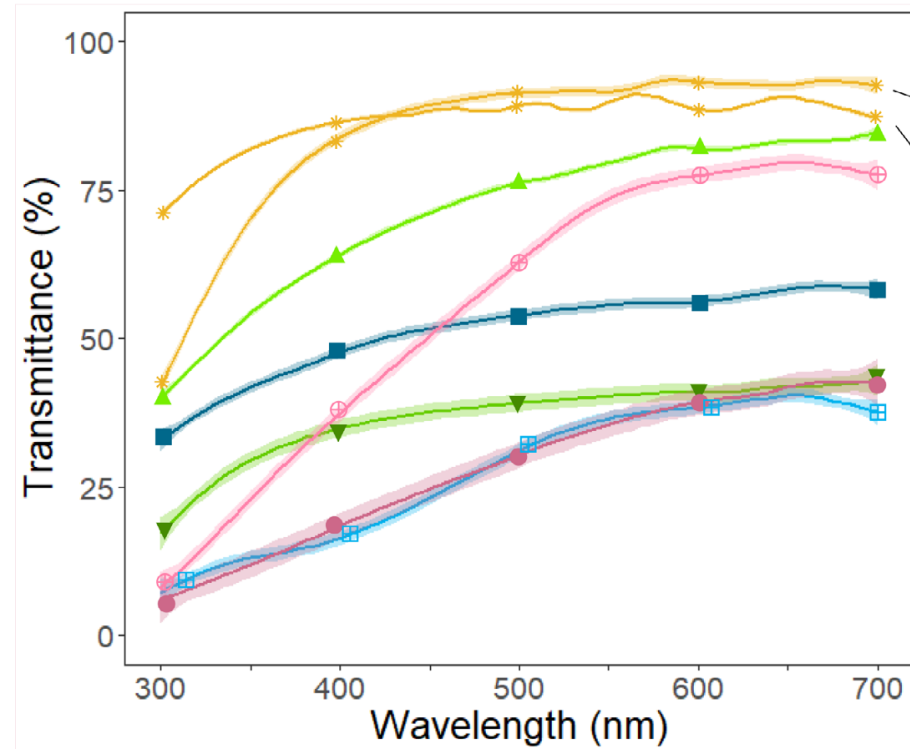

\section{Species}

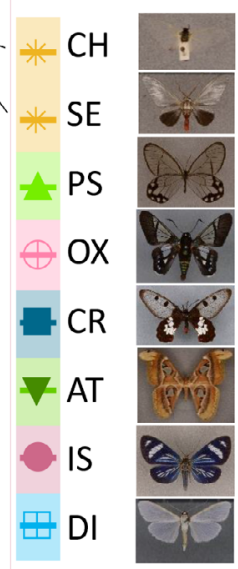

FIG. 2. (a) Relationship between the proportion of UV transmittance and the mean transmittance over 300-700 $\mathrm{nm}$. Species are represented by their forewing value. Perfect transparency (100\% transmittance over 300-700 nm, resulting in a 0.25 proportion of UV transmittance, upper right corner) is represented by an empty red square and species examples from Fig. 1 are indicated by their twoletter code inside the plot. Type is $\mathrm{P}$, piliform scales, or L, lamellar scales (the combination of piliform and lamellar scales was assimilated to lamellar scales); insertion is $\mathrm{E}$, erect, or F, flat; and coloration is $\mathrm{C}$, colored, or T, transparent. The $\mathrm{N}$ strategy has no scales, no insertion, and no coloration. The regression line was drawn from the best mixed model for the forewing. (b) Mean transmittance spectra of the five points of the forewing for the species listed in Fig. 1, with standard errors. Names are ordered from top to bottom according to decreasing transmittance values at $700 \mathrm{~nm}$. Small pictures (see Fig. 1 for details) of the species are displayed next to their abbreviated name. Colors and symbols for structural strategies (combination of scale type, insertion, and coloration) are the same in both plots.

(PLE, 5/123 species, 3/31 families). Transparent piliform scales were always associated with lamellar scales (PL) and never alone, be they erect or flat. Overall, colored scales $(\mathrm{P}, \mathrm{L}$, or $\mathrm{PL})$ concerned 72 species while transparent scales (L or PL) concerned 30 species.

\section{Evolution of structural features}

Phylogenetic signal and phylogenetic clustering.-We examined to what extent structural traits were influenced by common ancestry in their evolution. Wing 


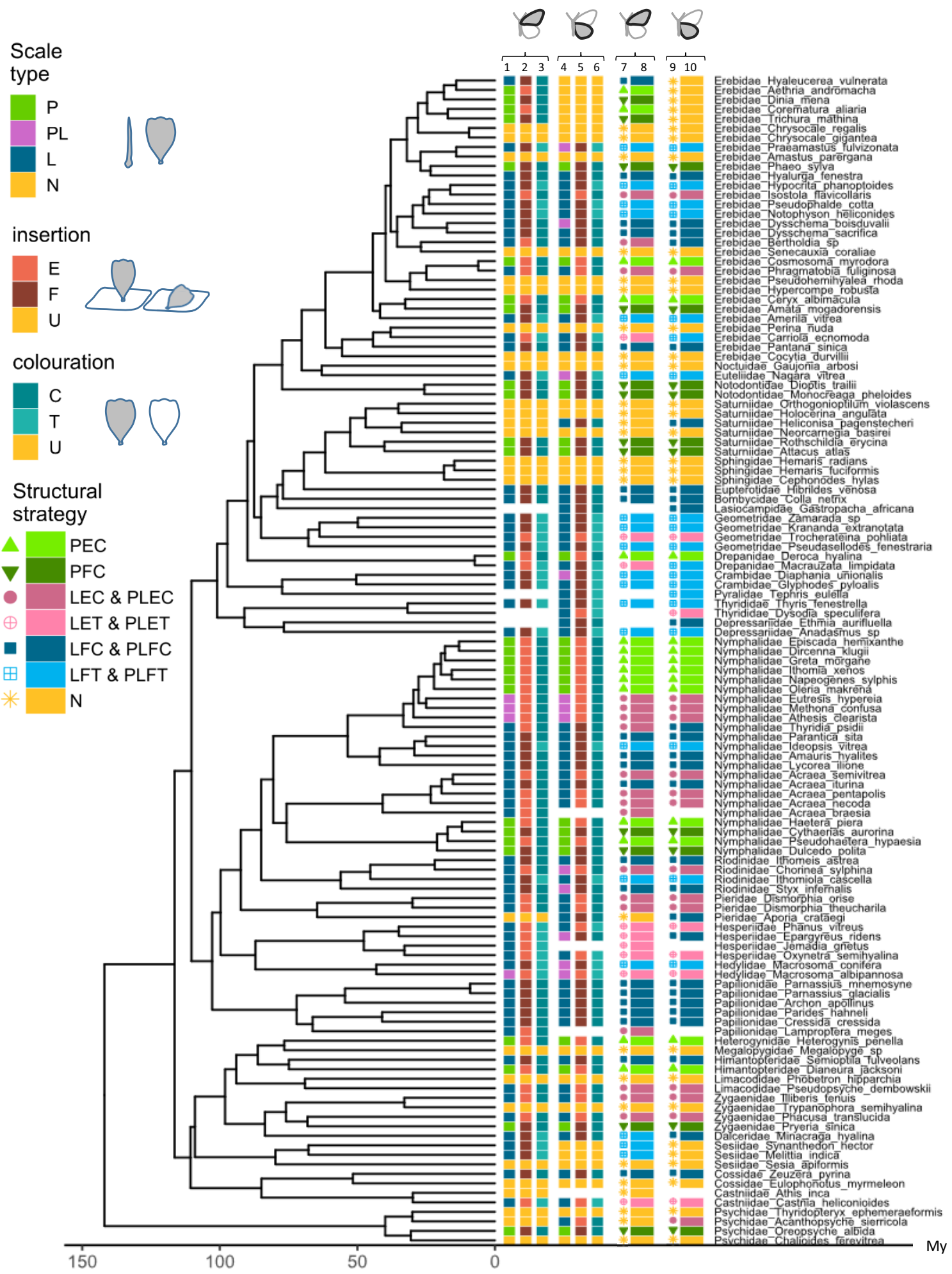

FIG. 3. Phylogeny of the species included in this study and distribution of scale structural traits and structural strategies in these species for the forewing (columns 1,2,3,7,8) and the hindwing (columns 4, 5, 6, 9, 10). A structural strategy is defined as the combination of scale type, insertion, and coloration. Type is $\mathrm{P}$, piliform scales, L, lamellar scales (piliform scales were assimilated to lamellar scales), or N, no scales. Insertion is $\mathrm{E}$, erect, F, flat, and $\mathrm{U}$, unknown (for $\mathrm{N}$ strategy). Coloration is $\mathrm{C}$, colored, T, transparent, and $\mathrm{U}$, unknown (for $\mathrm{N}$ strategy). The $\mathrm{N}$ strategy has no scales, no insertion, and no coloration. 
macrostructure (wing area, wing length, and clearwing area) showed significant phylogenetic signal for both forewing and hindwing (Appendix S1: Table S3). By contrast, optical variables of the transparent zone generally showed no phylogenetic signal, except the mean transmittance and the brightness contrast of the hindwing, which showed significant phylogenetic signal (but only for Pagel's $\lambda$ for the mean transmittance in the UV range; Appendix S1: Table S3).

At a microscopic level, scale dimensions and density showed significant phylogenetic signal in both transparent and opaque zones, for both forewing and hindwing (except for scale length in the opaque zone of the hindwing, Appendix S1: Table S3). Concerning binary structural variables, both wings showed the same evolutionary patterns (Appendix S1: Table S4): in the transparent zone, the evolution of scale insertion could fit both a Brownian motion model and a random model for the hindwing. In the transparent zone, scale presence, type, and coloration were conserved, i.e., they were significantly more clumped in the phylogeny than the Brownian Motion expectation. In the opaque zone, scale insertion was significantly more overdispersed than the random expectation, but this was likely due to little variation in that trait (1/123 species with different trait; Appendix S1: Table S4).

Several structural features showed significant phylogenetic clustering (i.e., species sharing a structural feature separated by fewer nodes than expected by chance). Specifically, in the transparent zone significant phylogenetic clustering was found on the forewing for the presence of scales, the presence of piliform scales alone or including the mixed category (PL, lamellar scales combined with piliform scales), for the erect insertion, and for the absence of transparency in scales (color C), and on the hindwing for the absence of scales and the presence of transparency in scales (Appendix S1: Table S5). In the opaque zone, there was significant clustering for the presence of lamellar scales alone (S) for the forewing, and the presence of combined piliform scales and lamellar scales (PL) for the hindwing. Considering structural strategies (i.e., the combination of given type, insertion and coloration), only $2 / 11$ appeared phylogenetically clustered in the transparent zone whatever the wing: erect colored piliform scales (PEC), erect colored lamellar scales mixed with piliform scales (PLEC), both strategies clustered when considering wings separately or together. For the hindwing, two structural strategies appeared clustered: the nude membrane $(\mathrm{N})$ and the flat transparent scales (LFT) (Appendix S1: Table S5). For the opaque zone, the structural strategy flat colored lamellar scales (LFC) was clustered for the forewing, while the structural strategy flat colored lamellar scales mixed with piliform scales (PLFC) was clustered for the hindwing.

Overall, structural strategies were less clustered phylogenetically than their composing structural parameters, scale type, insertion, and coloration. This suggested these structural parameters combined into structural strategies in multiple ways.

Correlated evolution between structural traits.-Analyses indicate that the structural strategy found in a zone (transparent or opaque) of one wing was likely to be found in the corresponding zone of the other wing. Scale presence, type ( $\mathrm{P}$ or $\mathrm{L})$, insertion, and color were correlated between wings in the transparent zone and scale type ( $\mathrm{P}$ or $\mathrm{L})$, and insertion were correlated between wings in the opaque zone (Appendix S1: Fig. S4 and Table S6). We found similar correlations in both wings: scale type (P or L) of the opaque zone was correlated with the scale type of the transparent zone. It was not the case for scale insertion. Scale type (P or L) and insertion were not correlated, in the transparent zone or the opaque zone, of the forewing or the hindwing. Conversely, in the transparent zone, scale coloration was correlated with scale type for the forewing and for the hindwing, and to scale insertion for the hindwing (Appendix S1: Fig. S4 and Table S6).

\section{Structural constraints and investment in transparency}

Analyses reveal some relationships between structural features that suggest the existence of evolutionary constraints.

At a macroscopic level, transparency occupied a larger proportion of wing area when it involved colored than transparent scales, especially in species with small wings (Table 1, Fig. 4a,c). Transparency concerned a larger proportion of wing area for smaller species (lower wing area); in other words, transparency could occupy a small or a large proportion of wing area in species with small wings, while transparency could only occupy a reduced proportion of wing area in species with large wings (Fig. 4a). These effects were stronger for the hindwing than for the forewing (Table 1, Fig. 4a).

At a microscopic level, we assessed the relative investment in transparency as the change in scale structure in the transparent zone compared to the opaque zone. As the wing area concerned by transparency increased, the difference in density between the transparent and the opaque zone decreased (Appendix S1: Table S7). As scale surface or coverage (whichever variable was retained depended on the dependent variable considered) increased, differences in scale density, length, width, surface or coverage between the transparent and opaque zones decreased (Appendix S1: Table S7). Compared to small species, larger species showed smaller changes in scale surface in the transparent zone compared to the opaque zone.

The relative investment in transparency depended on microstructural features. Compared to the opaque zone, the reduction in density in the transparent zone was greater for a nude membrane than for piliform scales $(\mathrm{P})$, and to a lesser extent greater for piliform scales than 
TABLE 1. Relationship between the proportion of clearwing area and wing macro- and microstructure.

\begin{tabular}{|c|c|c|c|}
\hline \multirow[b]{2}{*}{$\underline{\text { Factor }}$} & \multicolumn{2}{|c|}{ Mixed model } & \multirow{2}{*}{$\begin{array}{c}\text { Bayesian } \\
\text { Estimate [and 95\% CI] }\end{array}$} \\
\hline & Estimate $\pm \mathrm{SE}$ & $t$ & \\
\hline Intercept & $41.9 \pm 5.4$ & $7.74 * * *$ & $41.5[27.2,55.5]$ \\
\hline$(\mathrm{FW}>\mathrm{HW})$ & $-16.5 \pm 5.5$ & $-3.02 * * *$ & $-19.8[-30.1,-9.9]$ \\
\hline Coloration $(\mathrm{U}>\mathrm{C})$ & $0.15 \pm 2.75$ & 0.05 & $-1.6[-6.5,3.2]$ \\
\hline Coloration $(\mathrm{T}>\mathrm{C})$ & $-14.9 \pm 3.3$ & $-4.5 * * *$ & $-13.5[-19.9,-6.9]$ \\
\hline Clearwing area & $0.68 \pm 0.05$ & $15.05 * * *$ & $0.71[0.631,0.79]$ \\
\hline Wing length & $0.12 \pm 0.34$ & 0.34 & $0.39[-0.26,1.02]$ \\
\hline Wing area & $-0.26 \pm 0.03$ & $-7.46 * * *$ & $-0.23[-0.3,-0.16]$ \\
\hline Coloration $(\mathrm{U}>\mathrm{C}) \times$ clearwing area & $0.03 \pm 0.02$ & 1.8 & $0.028[-0.003,0.06] \mp$ \\
\hline Coloration $(\mathrm{T}>\mathrm{C}) \times$ clearwing area & $0.17 \pm 0.04$ & $4.51 * * *$ & $0.134[0.065,0.202]$ \\
\hline Wing length $\times$ clearwing area & $-0.01 \pm 0.001$ & $-10.63 * * *$ & $-0.012[-0.014,-0.01]$ \\
\hline Wing length $\times$ wing area & $0.004 \pm 0.0004$ & $8.39 * * *$ & $0.003[0.002,0.004]$ \\
\hline Wing length $\times(\mathrm{FW}>\mathrm{HW})$ & $1.43 \pm 0.41$ & $3.48 * *$ & $1.41[0.66,2.17]$ \\
\hline Clearwing area $\times(\mathrm{FW}>\mathrm{HW})$ & $\mathbf{0 . 0 5} \pm \mathbf{0 . 0 2}$ & $2.61 *$ & $0.059[0.021,0.098]$ \\
\hline Wing area $\times(\mathrm{FW}>\mathrm{HW})$ & $-0.08 \pm 0.02$ & $-3.74 * * *$ & $-0.075[-0.115,-0.033]$ \\
\hline Phylogenetic variance & - & - & $146.4[92.4,217.2]$ \\
\hline Residual variance & - & - & $59.3[45.6,76.6]$ \\
\hline
\end{tabular}

Notes: Retained classic mixed model and Bayesian phylogenetically controlled mixed model for the proportion of surface occupied by the clearwing area. We removed the two outliers (corresponding to the saturnid Attacus atlas) from the data analyzed. FW, forewing; HW, hindwing, scale coloration (T, transparent; C, colored; U, unknown [for absent scales]); -, not applicable for the analysis considered. Values in boldface type are statistically important factors associated with $95 \%$ CI excluding zero in Bayesian models, less important factors are associated to $90 \%$ CI excluding zero (symbol $\mathbf{I}$ ) and $P$ values $(* P<0.05 ; * * P<0.01$; $* * * * P<0.001)$ for the mixed model adjusted for type III sums of squares.

for lamellar scales (alone or in combination with piliform scales L or PL; Fig. 5). Scale length was reduced to zero for the nude membrane, and more reduced when scales were erect than flat, flat scales showing an increase in scale length (Fig. 5, Appendix S1: Fig. S5 and Table S7). The reduction in scale width or surface was maximal for the nude membrane, less important in piliform scales, and even less important in lamellar scales. Scale width was even increased in transparent lamellar scales compared to colored lamellar scales. Finally, strategies that involved transparent lamellar scales (alone or in combination with transparent piliform scales) showed an increase in scale surface, and an increase in scale coverage in the transparent zone compared to the opaque zone.

Overall, two trends appear: except for transparent lamellar scales, transparency was associated with a reduction in density and in scale dimensions and coverage, which was maximal for the nude membrane (Fig. 5, Appendix S1: Fig. S5). Transparent lamellar scales followed the reverse trend, with an increase in scale width, surface and coverage on the membrane compared to the opaque zone of the same wing and species (Fig. 5, Appendix S1: Fig. S5).

\section{Optical transparency and influence of structure}

Mean transmittance over (300-700 nm) was higher for the forewing than the hindwing; it was positively correlated with the proportion of UV transmittance (Fig. 2a) and this relationship did not differ between forewing and hindwing (Appendix S1: Table S8). However, a Pearson's correlation between mean transmittance over (300-700 nm) and proportion of UV transmittance yielded a moderate coefficient $(r=0.47$, $t=8.18, P<0.001$ ), suggesting that species can play on these aspects independently, to some extent.

At a macroscopic level, mean transmittance over (300-700 nm) and the proportion of UV transmittance were higher when transparency occupied a higher proportion of wing area and for smaller species (Fig. 4d, Table 2). In addition, mean transmittance was also higher for the forewing compared to the hindwing (Table 2), which was not caused by a higher proportion of clearwing area in the forewing as the proportion of clearwing area was higher for the hindwing than for the forewing (Table 1).

At a microscopic level, mean transmittance and the proportion of UV transmittance were higher for lower scale density, for a nude membrane than for erect scales and for erect scales than for flat scales, and for transparent scales than for colored scales (Figs. 6, 7, Table 2). Mean transmittance was higher for a nude membrane or a membrane covered with piliform scales than for a membrane covered with lamellar scales or a combination of lamellar and piliform scales, while the variation in the proportion of UV transmittance was not statistically important in relation to scale type, but only in relation to scale presence with nude membrane. Most effects depended on the wing considered and some effects (e.g. scale coloration, scale insertion $(U>E)$ ) were only statistically important when controlling for phylogeny, a 
a

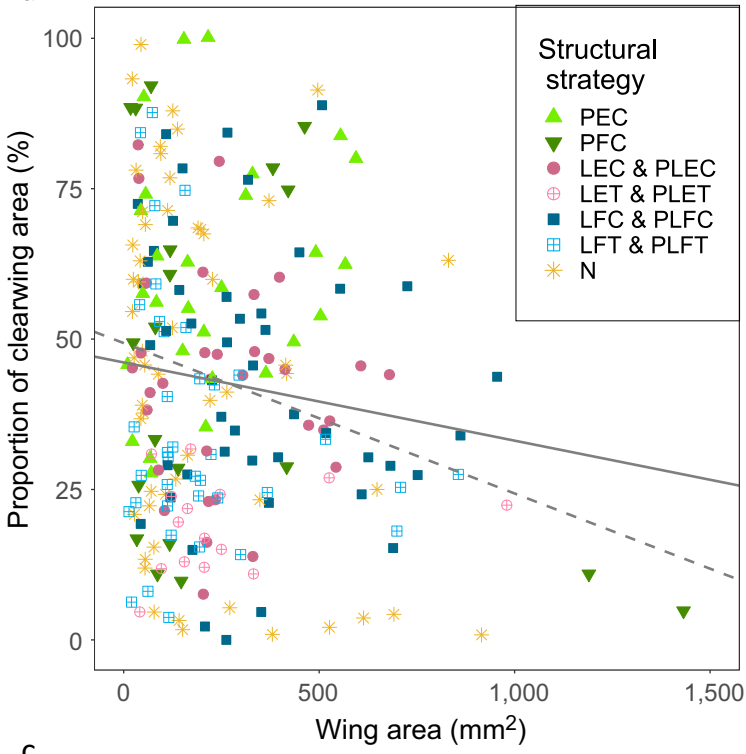

C

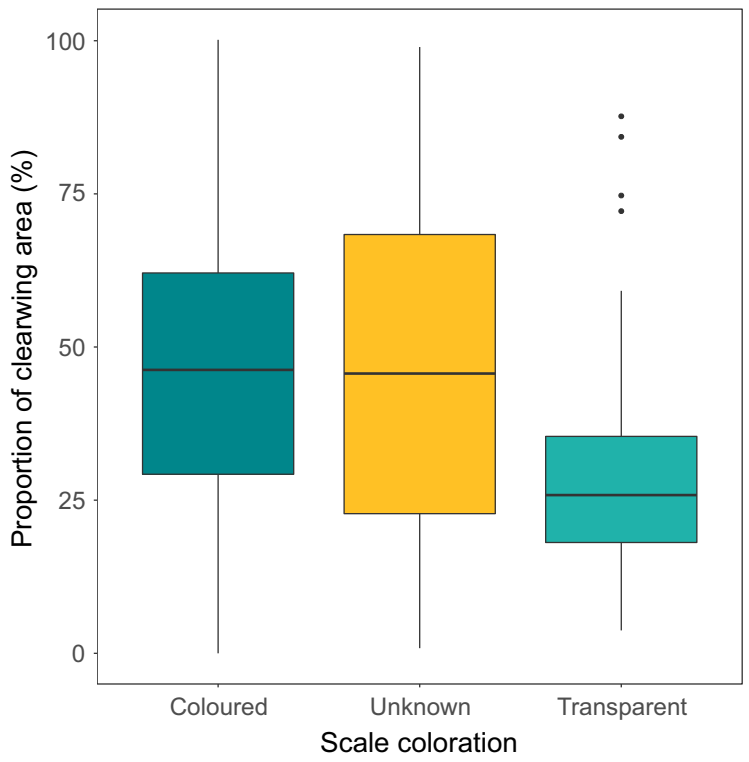

b
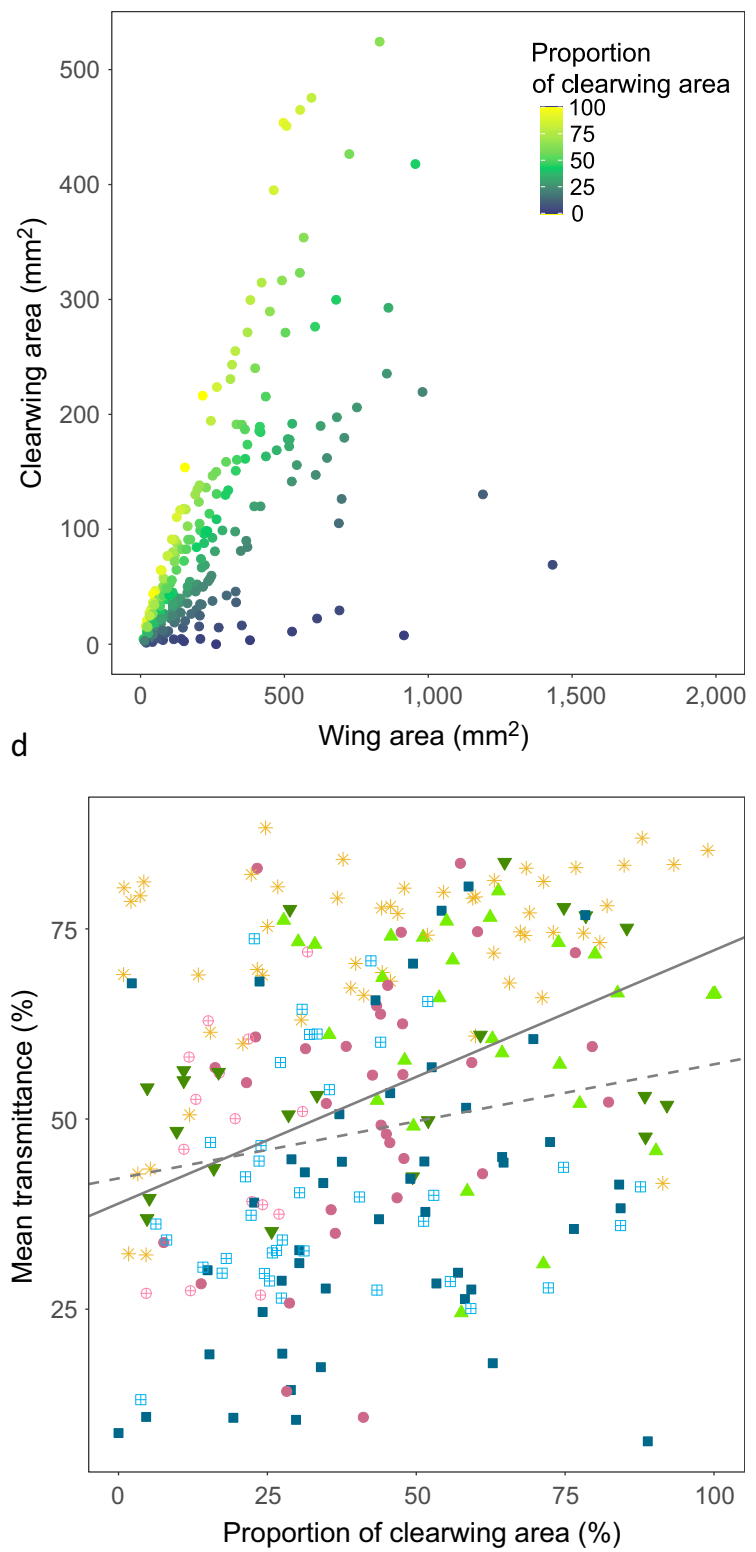

FIG. 4. Proportion of clearwing area (a) in relation to wing area and structural strategies, (b) in relation to clearwing area and wing area, and (c) in relation to scale coloration. (d) Mean transmittance over 300-700 nm in relation to the proportion of clearwing area and structural strategies. Lines were drawn from the coefficients of the best mixed model for the forewing (plain line) and the hindwing (dashed line). A structural strategy is defined as the combination of scale type, insertion, and coloration. Type is $\mathrm{P}$, piliform scales, or L, lamellar scales (the combination of piliform and lamellar scales were assimilated to lamellar scales); insertion is $\mathrm{E}$, erect, or F, flat; and coloration is $\mathrm{C}$, colored, $\mathrm{T}$, transparent, and $\mathrm{U}$, unknown (for $\mathrm{N}$ strategy). The $\mathrm{N}$ strategy has no scales, no insertion, and no coloration. The color codes of structural strategies, described in plot a, are identical in plot d. Species are represented by two points, one for forewing and one for hindwing. For clarity reasons, two points were removed from the plots shown in panels $\mathrm{a}$ and $\mathrm{b}$ and from analyses.

fact that may be explained by a high optical variation between closely-related species with similar microstructural features. The large variation in transmittance within each structural category shown in Fig. 6 suggests that many key features contribute to building the optical signal.
Overall, optical transparency, as estimated by the mean transmittance over $(300-700 \mathrm{~nm})$ and the proportion of UV transmittance, depended on both wing macrostructure and wing microstructure. It increased as it occupied a larger proportion of wing area and as membrane scale coverage decreased. 


\begin{tabular}{|c|c|c|c|c|c|c|c|}
\hline $\begin{array}{l}\text { Change in the } \\
\text { transparent zone } \\
\text { relative to the } \\
\text { opaque zone }\end{array}$ & $\mathrm{N}$ & PEC & PFC & LFC PLFC & LEC PLEC & PLET & LFT PLFT \\
\hline Change in density & Maximal decrease & Slight decrease & Slight decrease & No change & No change & Increase & Increase \\
\hline Change in length & Maximal decrease & No change & Increase & Increase & No change & No change & Increase \\
\hline Change in width & Maximal decrease & Decrease & Decrease & Slight decrease & Slight decrease & Increase & Increase \\
\hline Investment in pigments & Absent & Present & Present & Present & Present & Absent & Absent \\
\hline Investment in chitin & Absent & + & + & + & + & ++ & t++ \\
\hline
\end{tabular}

FIG. 5. Diagram showing the main results on relative investment in transparency detailed in Appendix S1: Table S7. Relative investment in transparency measures to what extent scale structural features in the transparent zone are modified relatively to the opaque zone, considering scale density, length, and width, as well as total investment in pigments and in chitin. Structural strategies are ranked from left to right from minimal investment to maximal investment. Notice that strategies based on transparent scales (be they lamellar scales alone or in combination with piliform scales) involve a relative investment higher than in the opaque zone. A structural strategy is defined as the combination of scale type, insertion and coloration. Type is P, piliform scales, or L, lamellar scales (the combination of piliform and lamellar scales was assimilated to lamellar scales); insertion is E, erect, or F, flat; and coloration is $\mathrm{C}$, colored, or $\mathrm{T}$, transparent. The $\mathrm{N}$ strategy has no scales, no insertion, and no coloration. The color codes of structural strategies are identical as in other figures.

\section{Ecological relevance of transparency}

Relevance for visual detection and concealment.-As expected, a greater optical transparency, estimated by mean transmittance or by proportion of UV transmittance, yielded a reduced color and brightness contrast between the transparent zone and the visual background, whatever the background (sky, leaf, trunk), the illuminant (woodland shade, forest shade, large gap) and the considered bird visual system (UVS or VS) (Appendix S1: Tables S9, S10, S11, S12). The positive interaction term between color and brightness contrasts was statistically important in all models, indicating that the reduction in visual contrast decreased as optical transparency increased (Fig. 8). Moreover, we found that mean transmittance was higher for the forewing compared to the hindwing, as in previous analyses (Table 2). For a given increase in mean transmittance, the reduction in brightness contrast was stronger for the forewing than for the hindwing (Appendix S1: Tables $\mathrm{S} 9, \mathrm{~S} 10)$. For a given increase in mean transmittance, the reduction in color contrast was stronger for the hindwing, whatever the background for the woodland shade, but only for the sky background for the forest shade and large gap ambient lights (Appendix S1: Tables S9, S10). Similar results were found for both bird visual systems.

Overall, a greater optical transparency yields greater concealment in various terrestrial backgrounds and lights, as perceived by bird predators.

Relevance for thermoregulation.-We found that mean transmittance in the near infrared range $(700-1,100 \mathrm{~nm})$ and in the human-visible range (400-700 nm) decreased with increasing latitude (Fig. 9c, d, Appendix S1: Table S13), a pattern that supported the hypothesis that transparent wing elements have an impact on thermoregulation. The mean transmittance in the UV range did not vary with latitude (Fig. 9a, Appendix S1: Table S13). Mean transmittance in the near infrared range $(700-1,100 \mathrm{~nm})$ correlated more tightly to transmittance in the human-visible range $(400-700 \mathrm{~nm})$ than to transmittance in the UV range $(300-400 \mathrm{~nm} ; 1,421$ AIC counts below; Appendix S1: comparing Fig. S6B and S6A). This was compatible with a greater thermal impact of infrared wavelengths. Such variations were independent of species size (factor not retained in the analyses).

Overall, mean transmittance decreases with increasing latitude, in the near infrared range and in the humanvisible range, supporting the hypothesis of a potential role of transparency in thermoregulation.

Relevance for UV protection. - All transmittance spectra showed a decrease in transmittance in the UV range, resulting in a proportion of UV transmittance lower than 0.25 (Fig. 2). This can be interpreted as potential UV protection. Yet, this hypothesis is not supported by the analysis of the patterns of UV variation with latitude. The mean transmittance in the UV range and the proportion of UV transmittance showed no significant variation with latitude (Fig. 9a, b, Appendix S1: Table S13). These results do not support the hypothesis that transparency offered UV protection, as an increase with latitude was expected for both variables.

\section{Discussion}

\section{Diverse structural strategies produce transparency}

Transparent or translucent wings are present in at least 31 families, representing a quarter of the extant Lepidoptera families, which collectively harbor a striking majority of opaque species. Transparency has thus likely evolved multiple times independently. With these 
TABLE 2. Relationships between mean transmittance over $(300-700 \mathrm{~nm})$ or the proportional UV transmittance and wing microstructure.

\begin{tabular}{|c|c|c|c|c|c|c|}
\hline \multirow[b]{3}{*}{ Factor } & \multicolumn{3}{|c|}{ Mean transmittance over $300-700 \mathrm{~nm}$} & \multicolumn{3}{|c|}{ Proportional UV transmittance $\dagger$} \\
\hline & \multicolumn{2}{|c|}{ Mixed model } & \multirow{2}{*}{$\begin{array}{l}\text { Bayesian estimate } \\
\text { [and } 95 \% \mathrm{CI} \text { ] }\end{array}$} & \multicolumn{2}{|c|}{ Mixed model } & \multirow{2}{*}{$\begin{array}{l}\text { Bayesian estimate } \\
\text { [and } 95 \% \mathrm{CI} \text { ] }\end{array}$} \\
\hline & Estimate $\pm \mathrm{SE}$ & $t$ & & Estimate $\pm \mathrm{SE}$ & $t$ & \\
\hline Intercept & $71.7 \pm 5.2$ & $13.88 * * *$ & $67.3[41.5,92.3]$ & $18.2 \pm 1.2$ & $15.24 * * *$ & $19.4[11.8,26.7]$ \\
\hline$(\mathrm{FW}>\mathrm{HW})$ & $10.8 \pm 3.1$ & $3.48 * * *$ & $9.3[6,12.7]$ & $1.4 \pm 0.5$ & $2.49 *$ & $1.6[1,2.2]$ \\
\hline Scale surface & - & - & - & $-0.00018 \pm 0.00008$ & $-2.15 *$ & $\begin{array}{l}-0.00016[-0.00025, \\
-0.00007]\end{array}$ \\
\hline Scale density & $-0.025 \pm 0.006$ & $-4.43 * * *$ & $\begin{array}{l}-0.01[-0.018 \\
-0.003]\end{array}$ & $-0.00208 \pm 0.00123$ & -1.69 & $\begin{array}{l}-0.002[-0.0034 \\
-0.00058]\end{array}$ \\
\hline $\begin{array}{l}\text { Scale type }(\mathrm{L}, \mathrm{PL})> \\
(\mathrm{P}, \mathrm{N})\end{array}$ & $-10.4 \pm 3.3$ & $-3.12 * *$ & $\begin{array}{l}-12[-17.6 \\
-6.4]\end{array}$ & $0.4 \pm 0.9$ & 0.39 & $-0.2[-1.4,1]$ \\
\hline Insertion $(\mathrm{F}>\mathrm{E})$ & $-10.1 \pm 2.5$ & $-4.09 * * *$ & $\begin{array}{l}-11.5[-14.7, \\
-8.1]\end{array}$ & $-2.7 \pm 0.6$ & $-4.69 * * *$ & $-3.3[-4,-2.6]$ \\
\hline Insertion $(\mathrm{U}>\mathrm{E})$ & $-4.3 \pm 3.6$ & -1.19 & $\begin{array}{l}-7.2[-12.2 \\
-2.3]\end{array}$ & $-2.3 \pm 0.8$ & $-2.82 * *$ & $-2.7[-3.7,-1.8]$ \\
\hline $\begin{array}{l}\text { Proportion of } \\
\text { clearwing area }\end{array}$ & $0.12 \pm 0.048$ & 2.49* & $\begin{array}{l}0.119[0.055 \\
0.182]\end{array}$ & $0.03736 \pm 0.00746$ & $5.01 * * *$ & $\begin{array}{l}0.03451[0.0262 \\
0.04269]\end{array}$ \\
\hline Clearwing area & $0.007 \pm 0.015$ & 0.45 & $\begin{array}{l}0.018[-0.004, \\
0.039]\end{array}$ & - & - & - \\
\hline Color $(\mathrm{T}>\mathrm{C})$ & $2.4 \pm 3.2$ & 0.74 & $8.8[4,13.6]$ & $0.9 \pm 0.8$ & 1.06 & $2.2[1.2,3.2]$ \\
\hline Wing length & $-0.2 \pm 0.1$ & $-2.01 *$ & $\begin{array}{l}-0.2[-0.4, \\
-0.1]\end{array}$ & $-0.02623 \pm 0.02232$ & -1.18 & $\begin{array}{l}-0.04793[-0.07705, \\
-0.01899]\end{array}$ \\
\hline $\begin{array}{l}(\mathrm{FW}>\mathrm{HW}) \times \\
\text { scale surface }\end{array}$ & - & - & - & $-0.0001 \pm 0.00007$ & -1.41 & $\begin{array}{l}-0.00011[-0.00019, \\
-0.00004]\end{array}$ \\
\hline $\begin{array}{l}(\mathrm{FW}>\mathrm{HW}) \times \\
\text { scale density }\end{array}$ & $-0.015 \pm 0.005$ & $-2.74 * *$ & $\begin{array}{l}-0.013[-0.019 \\
-0.007]\end{array}$ & $-0.00135 \pm 0.00111$ & -1.22 & $\begin{array}{l}-0.00156[-0.00271, \\
-0.00038]\end{array}$ \\
\hline $\begin{array}{l}(\mathrm{FW}>\mathrm{HW}) \times \\
\text { scale type } \\
(\mathrm{L}, \mathrm{PL})>(\mathrm{P}, \mathrm{N})\end{array}$ & $2.9 \pm 2.4$ & 1.21 & $4[1.5,6.4]$ & $0.7 \pm 0.5$ & 1.37 & $0.7[0.2,1.1]$ \\
\hline $\begin{array}{l}(\mathrm{FW}>\mathrm{HW}) \times \\
\text { insertion } \\
(\mathrm{F}>\mathrm{E})\end{array}$ & $1.4 \pm 2.1$ & 0.67 & $0.5[-1.7,2.8]$ & $-0.9 \pm 0.4$ & $-2.29 *$ & $-0.9[-1.3,-0.5]$ \\
\hline $\begin{array}{l}(\mathrm{FW}>\mathrm{HW}) \times \\
\text { insertion } \\
(\mathrm{U}>\mathrm{E})\end{array}$ & $-8.7 \pm 3.3$ & $-2.61 *$ & $\begin{array}{l}-7.9[-11.5 \\
-4.6]\end{array}$ & $-1.8 \pm 0.6$ & $-3.04 * *$ & $-1.9[-2.5,-1.3]$ \\
\hline $\begin{array}{l}(\mathrm{FW}>\mathrm{HW}) \times \\
\text { clearwing } \\
\text { area }\end{array}$ & $-0.031 \pm 0.011$ & $-2.89 * *$ & $\begin{array}{l}-0.03[-0.042 \\
-0.018]\end{array}$ & - & - & - \\
\hline $\begin{array}{l}(\mathrm{FW}>\mathrm{HW}) \times \\
\text { color } \\
(\mathrm{T}>\mathrm{C})\end{array}$ & $-6.2 \pm 2.5$ & $-2.44 *$ & $-6.6[-9.2,-3.9]$ & $-0.4 \pm 0.5$ & -0.69 & $-0.5[-1,0.1]$ \\
\hline $\begin{array}{l}\text { Phylogenetic } \\
\text { variance }\end{array}$ & - & - & $\begin{array}{l}786.8[593.9 \\
1035.9]\end{array}$ & & & $72.1[55.8,93.2]$ \\
\hline $\begin{array}{l}\text { Residual } \\
\text { variance }\end{array}$ & - & - & $\begin{array}{l}55.3[50.8 \\
60.4]\end{array}$ & & & $1.9[1.7,2.1]$ \\
\hline
\end{tabular}

Notes: Best classic mixed model and Bayesian phylogenetically controlled mixed model for mean transmittance over 300-700 nm and proportion of UV transmittance (multiplied by 100 for the analysis). For all analyses, we used all 10 measurements per species. We treated species and wing within species as random factors in classic mixed models and species as a random factor in Bayesian models. A dash (-) indicates factors not retained in the best model or of no concern (for phylogenetic and residual variances). FW, forewing; HW, hindwing. Scale type, P, piliform scales; L, lamellar scales; PL, piliform scales and lamellar scales in combination; $\mathrm{N}$, none. Scale insertion on the wing membrane, U, unknown (for absent scales); F, flat; E, erect. Scale coloration T, transparent; $\mathrm{C}$, colored. All factors relating to scales concerned scales from the transparent zone. Values in boldface type are statistically important factors associated with $95 \%$ CI excluding zero in Bayesian models, less important factors are associated to $90 \%$ CI excluding zero (symbol $\mathbf{I}$ ) and $P$ values $(* P<0.05 ; * * P<0.01 ; * * * * P<0.001$ ) for the mixed model adjusted for type III sums of squares.

$\dagger$ The proportion of transmittance was multiplied by 100 before analysis.

multiple evolutions comes a massive diversity of structural strategies (combination of scale type, insertion, and coloration), expanding the range of strategies reported in the literature in the scarce studies conducted so far in clearwing Lepidoptera.
Counterintuitively, the most common structural strategy for transparency is not a nude membrane but flat lamellar scales, either colored and in lower densities or transparent and packed in high densities. While flat colored lamellar scales have been recorded in the literature 


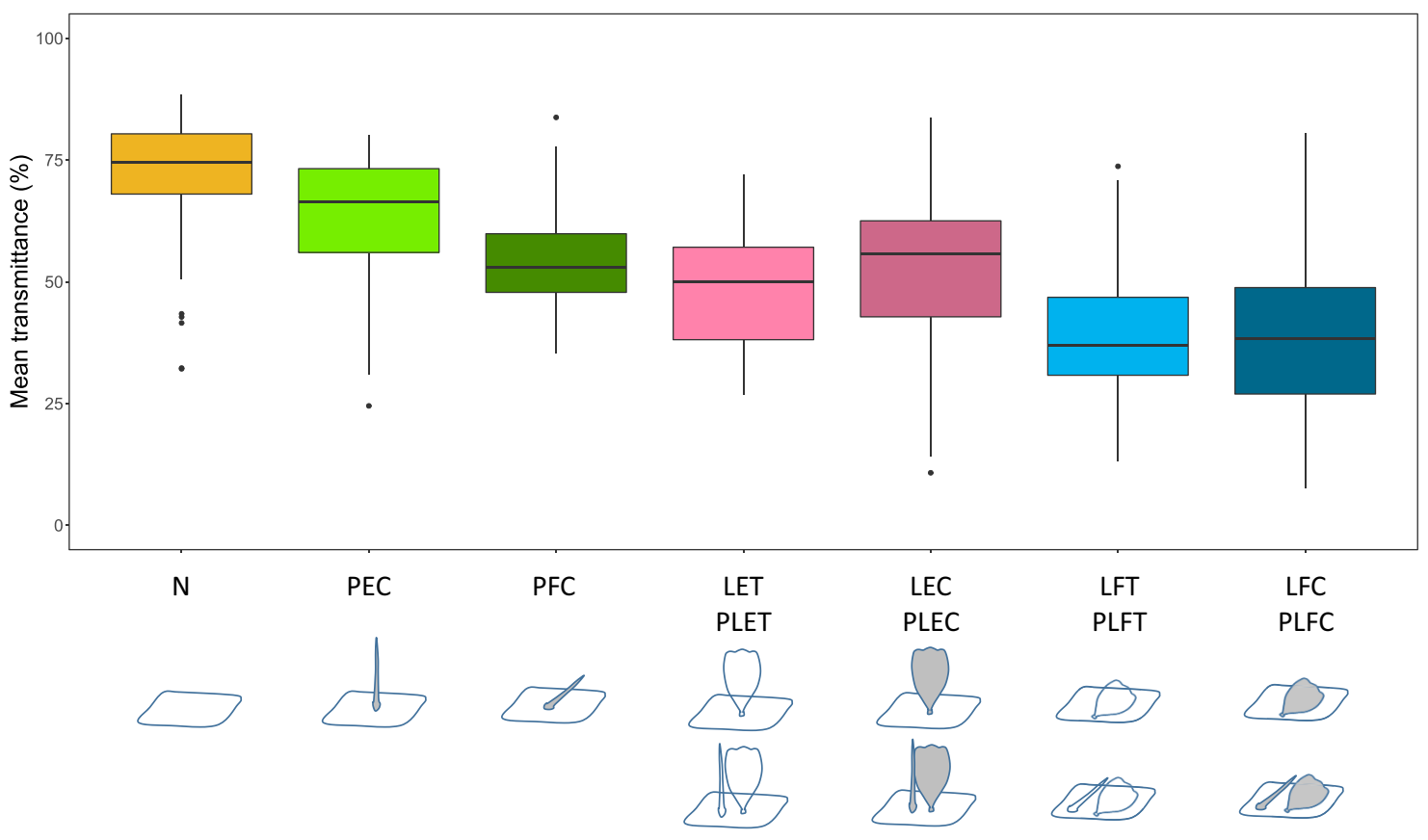

FIG. 6. Variations of transparency (mean transmittance) with structural strategies. A structural strategy is defined as the combination of scale type, insertion, and coloration. Type is $\mathrm{P}$, piliform scales, or L, lamellar scales (the combination of piliform and lamellar scales was assimilated to lamellar scales); insertion is E, erect, or F, flat; and coloration is C, colored, or T, transparent. The N strategy has no scales, no insertion, and no coloration. Mean transmittance was computed over 300-700 nm; for the graph, we took one average per wing and species.

(in the nymphalid Parantica sita and the papilionid Parnassius glacialis Goodwyn et al. 2009), flat transparent lamellar scales (LFT) have not been recorded in any clearwing Lepidoptera species so far, although they have been recorded in the opaque colored papilionid Graphium sarpedon (Berthier 2007, Stavenga et al. 2012). The nude membrane, already recorded in the sphingid Cephonodes hylas (Yoshida et al. 1997), can be fully nude (one-third of cases) or have scale sockets (twothirds of cases), which are the remnants of fully developed scales shedding at first flight, as in the sphingid genus Hemaris (Schmidt 2018, Koshkin and Yevdoshenko 2019), in Cephonodes hylas (Yoshida et al. 1996, 1997) and in Heliconisa pagenstecheri (Zikán 1927, Farina 2001). Piliform scales alone have been previously reported in the saturniid Rothschildia lebeau (Hernandez-Chavarria et al. 2004) or the nymphalids Greta morgane oto (Binetti et al. 2009, Siddique et al. 2015) and Cithaerias menander (Berthier 2007). The term "piliform scale" is an umbrella term that comprises monofid (bristle-like) scales and/or bifid (forked-like) scales, two types that may correspond to modifications of cover and ground scales, respectively. Indeed, both types can be found in the same transparent zone, for instance in Ithomiini species and in the drepanid Deroca hyalina. Moreover, when combined with lamellar scales, piliform scales are always monofid, which suggests they could be modified cover scales, a hypothesis that should be tested by distinctive marking of cover and ground scales during development. Piliform scales are never transparent when alone (no strategy PFT or PET), which likely results from structural or functional constraints. Finally, combined piliform scales and lamellar scales (PL) represents the rarest strategy in our data set (12 species, seven families), with erect colored piliform scales and lamellar scales (PLEC) showing a significant phylogenetic clustering. Another rare structural strategy involves transparent erect lamellar scales (LET, nine species, six families), which show no phylogenetic clustering, indicating that it evolved independently in distinct lineages. This is the first record of this LET structural strategy in the literature.

Overall, structural strategies seem less clustered phylogenetically than scale type, insertion, and coloration, suggesting that structural parameters combine into structural strategies in multiple ways. Yet, strategies are similar between forewing and hindwing, suggesting that they arise from genetic and physiological pathways that are implemented in both wings during development.

\section{Investment in transparency differs between structural strategies}

Analyses on relative investment show that structural strategies imply joint changes in different structural features. On the one hand, some strategies follow the general intuitive picture of transparency consisting of a "reduction" in scale density, dimensions, membrane coverage. 
a
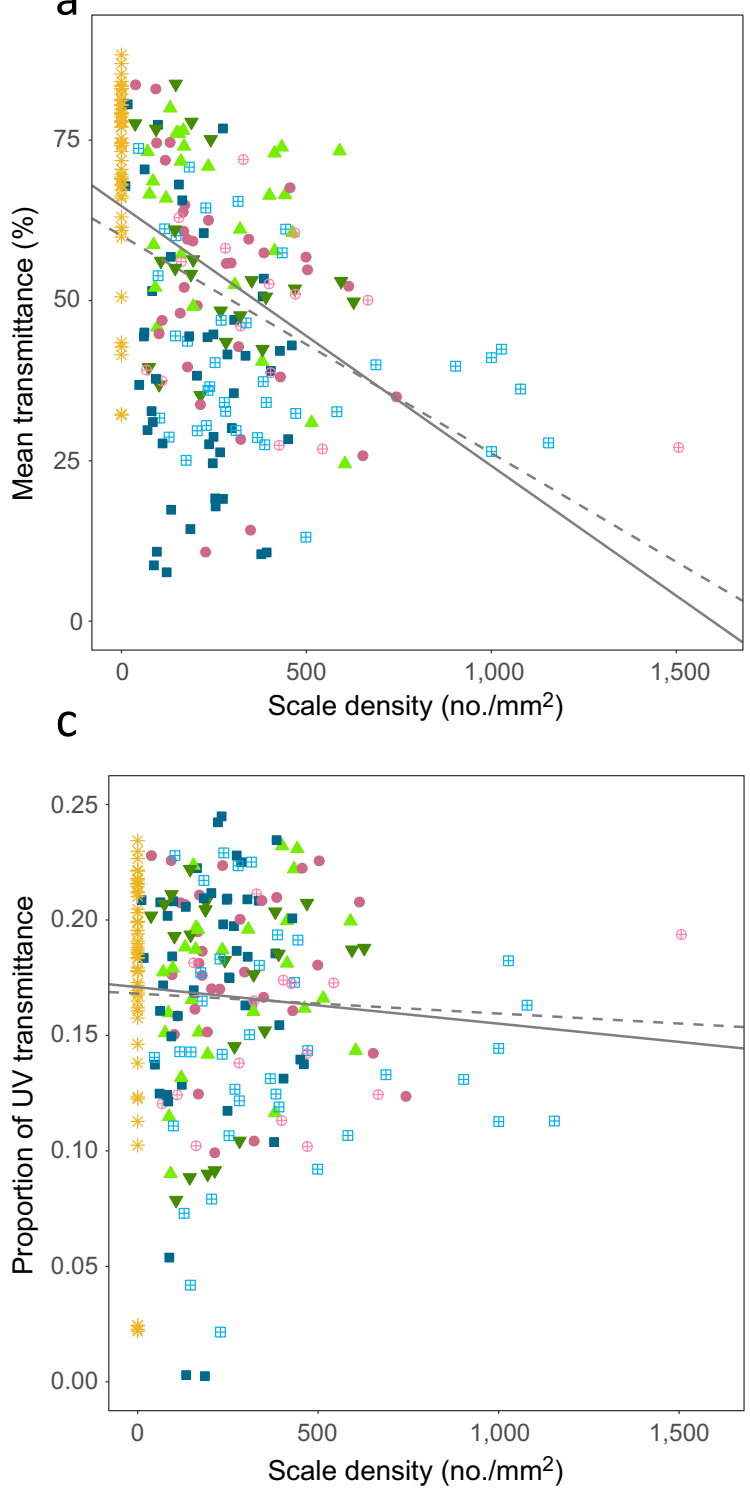

b
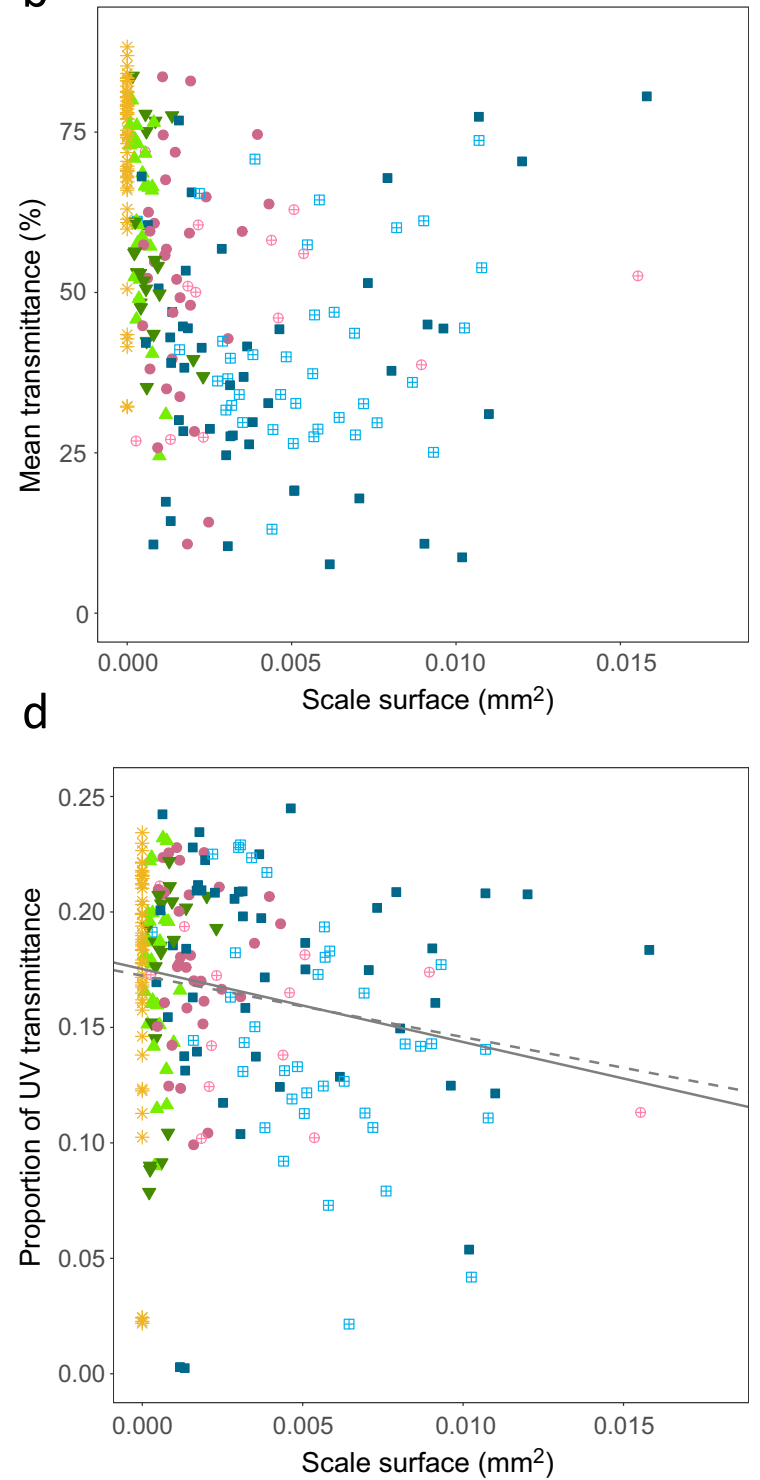

FIG. 7. Relationship between optical transparency, estimated either by (a, b) mean transmittance over 300-700 nm or (c, d) the proportion of UV transmittance, and (a, c) scale density or (b, d) scale surface. Species are represented by two values, one for forewing and one for hindwing. Lines were drawn from the coefficients of the best mixed model for the forewing (plain line) and the hindwing (dashed line). In panels $\mathrm{b}$ and $\mathrm{d}$, for clarity reasons, two points were removed from the graph but not from the analyses. A structural strategy is defined as the combination of scale type, insertion, and coloration. Type is P, piliform scales, or L, lamellar scales (the combination of piliform and lamellar scales was assimilated to lamellar scales); insertion is E, erect, or F, flat; and coloration is $\mathrm{C}$, colored, or $\mathrm{T}$, transparent. The $\mathrm{N}$ strategy has no scale, no insertion, and no coloration.

Reduction is apparently maximal for the nude membrane, although the initial investment can be high when scales fully develop and shed subsequently. Piliform scales (always colored when alone) are longer (especially when flat), thinner and in lower density than scales in the opaque zone, resulting in a low membrane coverage. Reduction seems minimal for colored lamellar scales, which are slightly thinner than their opaque counterparts, and associated with a slight decrease in scale surface and wing coverage. On the other hand, strategies based on transparent lamellar scales show an increased investment in chitin compared to the opaque zone. Wider and shorter (when erect) or wider and longer (when flat), transparent lamellar scales increase in surface and are more densely packed. We show clear correlations between scale coloration (colored vs. transparent) and scale morphology; while such correlations have been observed in opaque butterflies (e.g., Janssen et al. 2001, Matsuoka and Monteiro 2018) and 


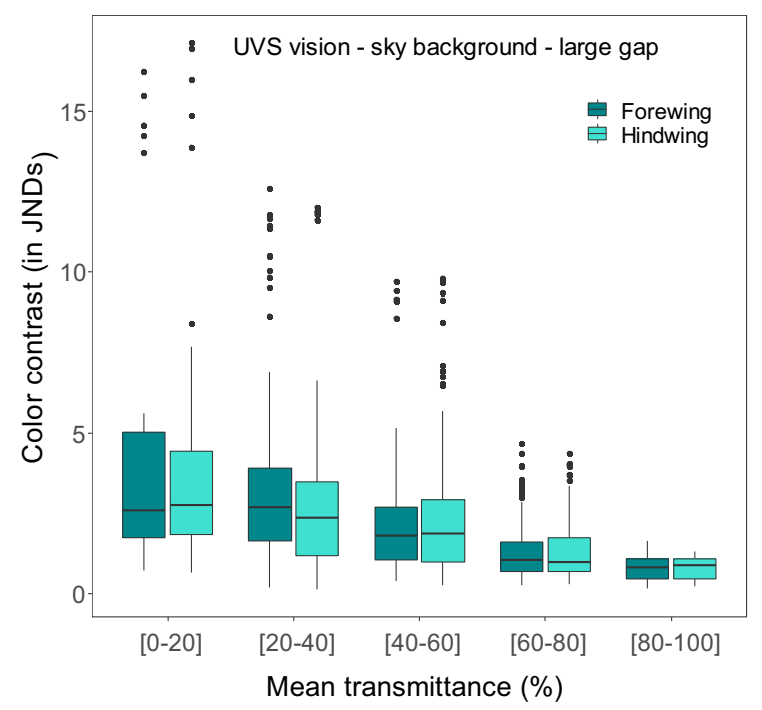

C

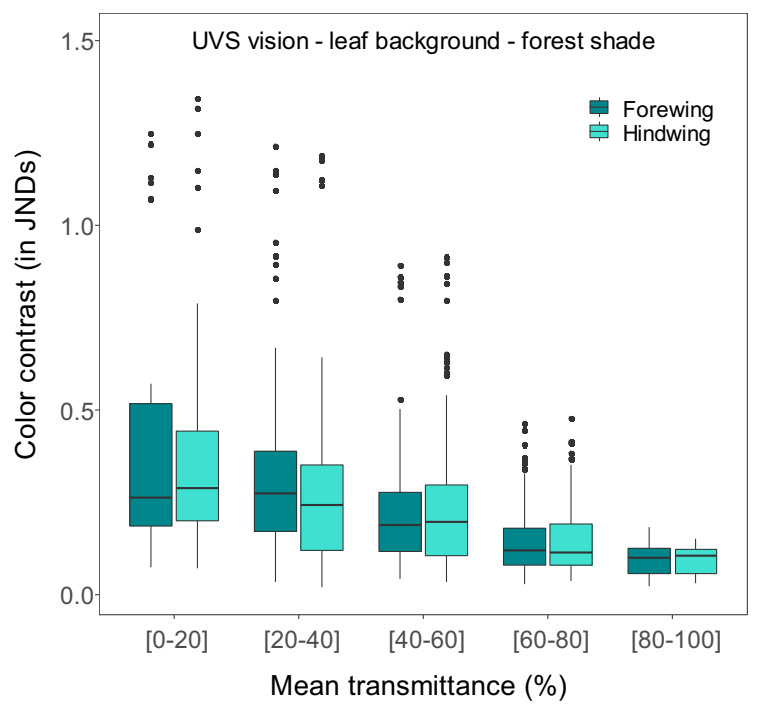

b

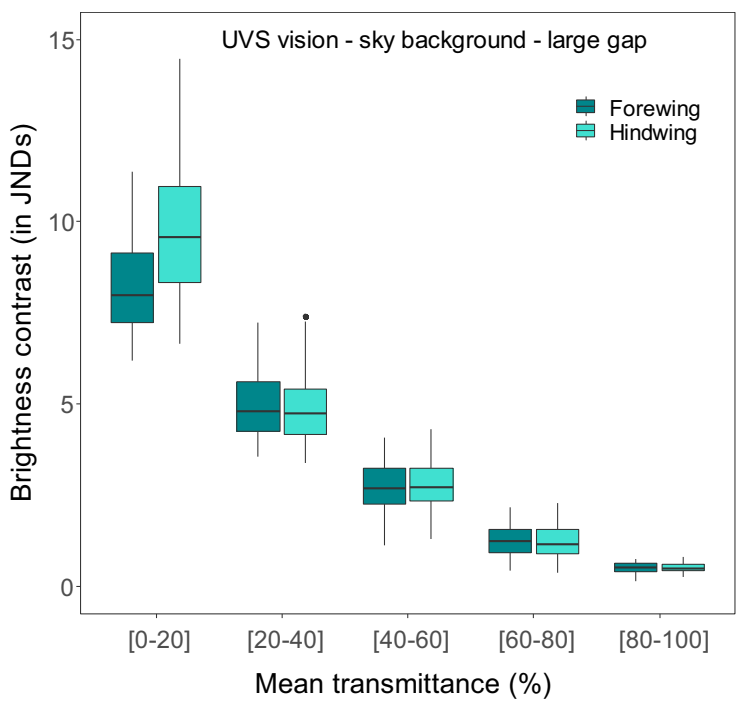

d

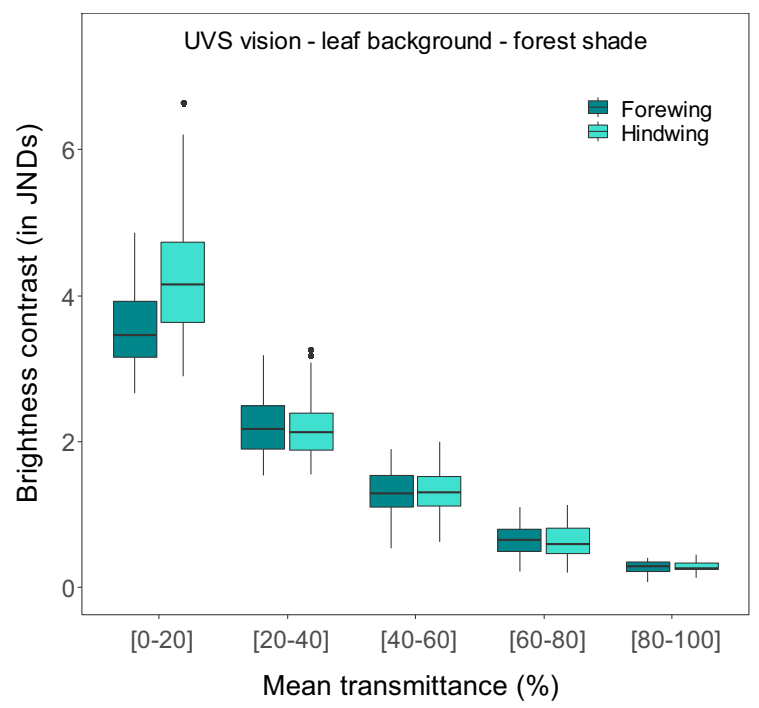

FIG. 8. Relationships between mean transmittance as an optical property and (a, c) color or (b, d) brightness contrasts, for the forewing (dark turquoise) and the hindwing (light turquoise). Color and brightness contrast values were obtained from perceptual vision modeling (see Methods for details) on all transmittance measurements and are expressed in JNDs, just discriminable differences. We show two examples of viewing conditions: a clearwing patch seen against the sky and under a large gap ambient light (a, b) or seen against a green vegetation background and under a forest shade ambient light (c, d), by a UVS vision bird predator. Detailed conditions and results are presented in Appendix S1: Figure S3 and Table S9 to S11, respectively.

investigated in genetics and development, this remains to be done in the case of transparency. If we use scale coverage to estimate the investment in chitin, transparency seems maximally economical for a nude membrane without sockets, whereas it seems costlier than the opaque zone when transparency is achieved through transparent scales. But in the latter case, the lower investment in pigment for these transparent scales may compensate the higher investment in chitin. While transparency can be intuitively thought as economical because based on an absence of scale, it is far from the case. Some strategies (like LE or LF) require more material overall than the opaque zone of the same wing and even the nude membrane can result from scales fully developing before shedding. Transparency may thus be costlier than classically viewed and future studies should quantify the energetic costs of transparency during development.

Patterns common to different species emerge, which may help to reveal structural constraints. For instance, transparent lamellar scales are packed in higher densities 


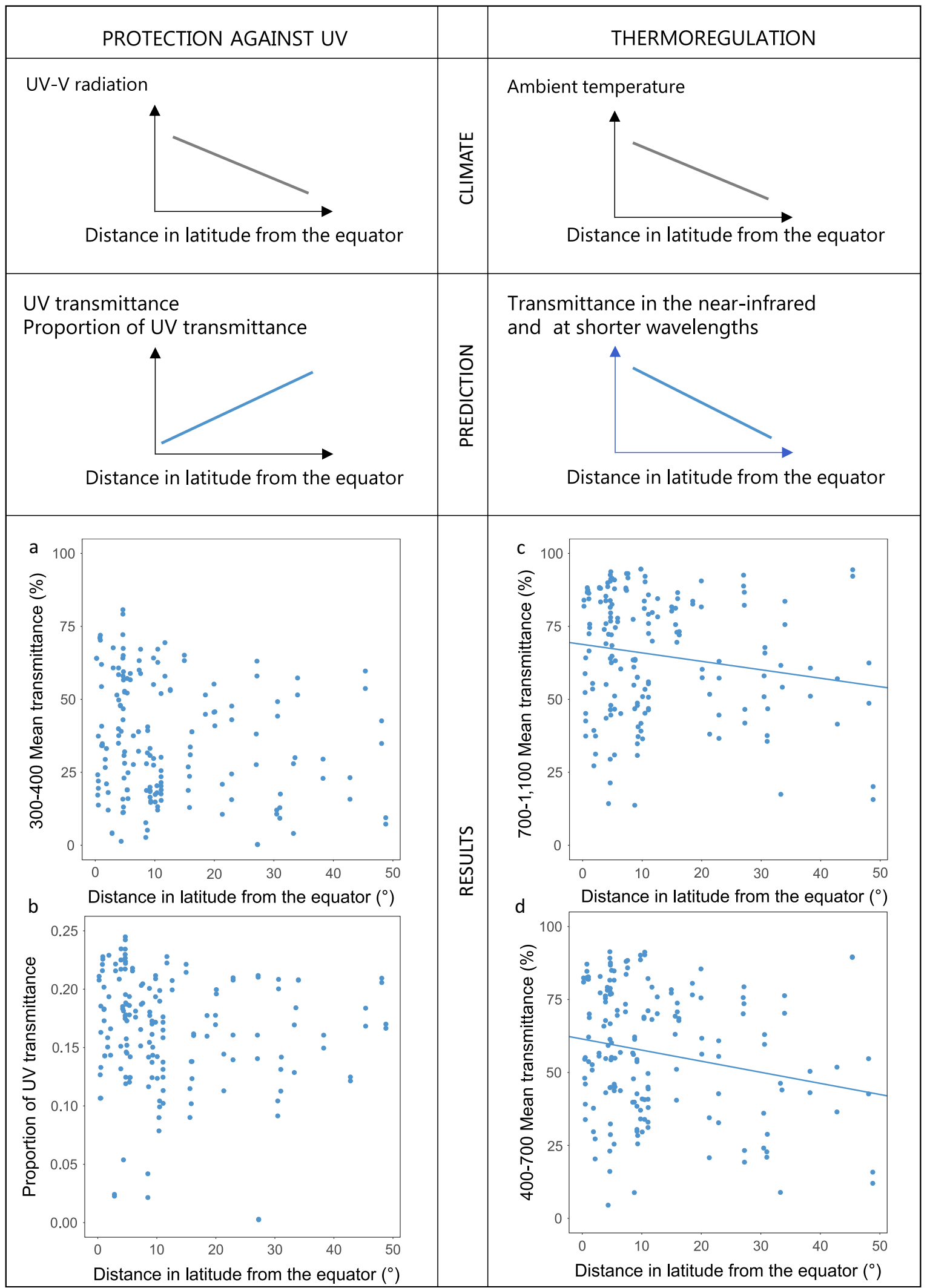


FIG. 9. Tests of the potential role of wing transparency as a protection against UV radiation (left column) and in thermoregulation (right column). Left column: UV-B radiation decreases as distance increases from the equator (Beckmann et al. 2014), and so does the need for UV absorption. We thus predict an increase in transmittance in the ultraviolet 300-400 nm range, or at least in the proportion of UV transmittance with increasing distance from the equator. Corresponding results are shown in left column (a, b). Right column: Ambient temperature decreases as distance increases from the equator. Absorbing radiation helps thermoregulation at cold temperatures. We thus predict a decrease in transmittance in the near-infrared $700-1,100 \mathrm{~nm}$ range and at shorter wavelengths, such as in the 400-700 range or even in the UV range with increasing distance from the equator. Corresponding results are shown in right column (c, d). Lines were drawn from the coefficients of the best mixed models. For clarity reasons, we plotted only two points per species, forewing and hindwing mean transmittance values but models were run on all points.

and broadened, especially when erect. Why so? We can invoke two non-mutually exclusive reasons. First, melanization may enhance mechanical resistance, as suggested in birds (Butler and Johnson 2004) and in Lepidoptera (only mentioned as personal communication in Brakefield 1987). In insects, cuticle melanization and cuticle hardening (sclerotization) occur with little time lag during development and share very similar pathways with common precursors and some sclerotization components provide different coloration in insect cuticles, from colorless to yellow and brown (Sugumaran 2009). Knockout mutations in genes that function in the melanin pathway affect both scale coloration and scale fine structure (Matsuoka and Monteiro 2018). Shortening melanin-deprived transparent scales may be a way to reinforce their mechanical resistance; it would be interesting to explore whether these shape changes come with fine structural changes that reinforce scale mechanical resistance. This may also explain why transparent piliform scales are absent from our data set, as long and thin melanin-deprived piliform scales may be too fragile. Second, shortening erect lamellar scales and enlarging them (see the hesperid Oxynetra semihyalina in Fig. 1) may be beneficial for water repellency and mechanical resistance. Erect scales (PE piliform scales alone, LE lamellar scales alone or PLE, the combination of piliform scales and lamellar scales) provide hierarchically structured geometry with asperities and air pockets between them. Such a multiscale roughness is crucial to ensure superhydrophobicity, as shown both in theoretical studies (Nosonovsky and Bhushan 2007) and in natural systems, such as the cuticle of water striders (Goodwyn et al. 2008).

\section{Structural strategies differ in their optical efficiency}

Optical properties of transparency evolved more rapidly than structures, as shown by the absence of phylogenetic signal in most color descriptors. Structural strategies are correlated with their efficiency at transmitting light. The nude membrane category is the most efficient while lamellar scales alone or in combination with piliform scales are the least efficient. Erect scales are more efficient than flat scales, transparent are more efficient than colored scales. Yet, the large variation in transmittance within structural strategies indicates that other parameters are crucial: scale density, dimension and surface are key features. The lower the density, scale surface and coverage on the wing membrane, the higher the transmittance. Additional parameters not measured here, e.g., the pigmentation of scales and of the wing membrane, the dimension and density of nanostructures, likely play a role, as shown in recent studies (Pinna et al. 2020, Pomerantz et al. 2021). For instance, wing nanostructures like nanopillars found in the glasswing nymphalid Greta morgane oto create by their very shape a progressive air-chitin gradient from the air interface toward the chitinous membrane that facilitates light transmission and reduces light reflection, acting as effective antireflective devices (Binetti et al. 2009, Siddique et al. 2015). Both the density and shape of the nanorelief are crucial to determine the amount of reduction of light reflection.

\section{Ecological relevance of transparency for visual detection, differences between small and large species}

Increasing transmittance reduces the visual contrast both brightness and color contrast - offered by Lepidoptera with various visual backgrounds (sky, leaf, trunk), in various light conditions encountered in closed or open habitats, and as seen by two examples of tetrachromatic visual systems. This underlines the potential protective role offered by transparency on land and illustrates the principle of transparency, namely giving access to the visual background, whatever the illuminant, the background and the visual system. The reduction in visual contrast gets lower as mean transmittance increases, which may explain why mean transmittance never exceeds $90.25 \%$ in our data set. While the translation of mean transmittance into brightness contrast seems intuitive, the translation of mean transmittance into color contrast is more surprising and underlines that transmittance spectra are not achromatic. Indeed, even if transmittance can reach up to $95 \%$ in the $(400$ $700 \mathrm{~nm}$ ) range, it is lower in the UV range, which can be due either to diffuse transmittance or to absorption by molecules, such as chitin or pigments, in the scales or the wing membrane. Chitin shows higher absorption at short than long wavelengths (Azofeifa et al. 2012, Stavenga et al. 2014). Melanin has a higher specific absorption at short than at long wavelengths (Wolbarsht et al. 1981) and even a weak pigmentation produces a loss of transmittance in the UV range first.

Are there selective processes that could explain the lower transmittance in the UV range? It can result from 
non-mutually exclusive reasons: first, weaker selection by visually hunting predators in this range, where they are less sensitive (low number of UVS or VS sensitive cones in the retina of birds, leading to a higher relative noise, as shown in Vorobyev and Osorio [1998]). Second, wavelengths in the UV range are cut out by vegetation (Endler 1993), thereby attenuating the importance of this range for species living in closed habitats. Third, the need for communication or thermoregulation (see the last two sections of the Discussion) may offset the visual gain brought by an increase in transmittance in the UV range. Species can exploit the UV range for communication with conspecifics, as suggested in opaque species (Bybee et al. 2012).

Interestingly, our analyses reveal size-dependent strategies that can be interpreted in the context of a role of transparency in visual detection and concealment. (1) In small species (with low values of wing area), transparency spans a wide range of proportions of wing area, from spots to entire wing, but in large species (with high values of wing area), transparency is always restricted to small proportion of wing surface. (2) The efficiency of transparency at transmitting light positively correlates with the proportion of clearwing area. Hence, small and poorly transmitting spots can be present in wings of all species, such as the large saturniid Rothschildia lebeau (Hernandez-Chavarria et al. 2004) and the small thyridid Dysodia speculifera, but almost entirely transparent wings highly efficient at transmitting light can only be found in small species, such as the psychiid Chalioides ferevitrea, the wings of which are extremely difficult to detect. Why so? For small species, having large proportions of clearwing area can be profitable given their overall small size and low detectability. Conversely, large species may not benefit from high proportions of clearwing area, for several non-mutually exclusive reasons.

First, optical benefits of having large transparent areas may be limited in large species. Kang et al. (2017) have found in erebid moths that small species have cryptic coloration in both wings, while large species have cryptic coloration on the forewing, and hidden contrasting color signals on the hindwing, probably involved in startle displays. They experimentally show that crypsis fails as body size increases and secondary defenses like startle displays are more effective in large prey. If such an anti-predator switch applies to transparency, crypsis by the means of transparency could potentially fail in large species for two reasons: a conspicuous large opaque body, and a wing transparency too limited in efficiency by wing membrane thickness. More studies are needed to test whether such a size-dependent switch of transparency in wing pattern exists and to unravel its structural determinants.

Second, optical benefits may be offset by costs entailed by large transparent areas for other functions. Among the Lepidoptera species investigated so far (Wagner et al. 1996, Zheng et al. 2007, Goodwyn et al. 2009, Wanasekara and Chalivendra 2011, Fang et al.
2015), large species like the nymphalid Parantica sita and the papilionid Parnassius glacialis show moderate to high hydrophobicity (Goodwyn et al. 2009, Fang et al. 2015). Conversely, the species with the highest proportion of clearwing area, the nymphalid Greta oto, has one of the lowest hydrophobicity values found (Wanasekara and Chalivendra 2011). The structural determinants of hydrophobicity in clear wings remain to be identified, and potential trade-offs between optics and hydrophobicity should be investigated.

Furthermore, what are the potential benefits for large species to have small transparent spots, which sometimes account for only a few percent of wing total area? We can propose several hypotheses: (1) clear spots can create false margins, resulting in internal edges more salient than the animal true outline that function as surface disruption patterns (Stevens et al. 2009b). As suggested by Costello et al. (2020), these false internal edges can create false holes, especially when edges are enhanced; this creates false depth planes and incorrect visual categorization as depth cues. (2) Clear-spotted, cryptically colored Lepidoptera species may be protected by masquerade, predators taking them for non-edible objects of their environment, like damaged or rotten leaves, an hypothesis formulated by Janzen (1984) but never tested. (3) When transmission is poor, small spots could resemble white-pigmented spots, which could be involved in communication. More generally, small clear spots may function as eyespots, which are efficient at limiting predator attack and divert them from vital parts (Stevens et al. 2008, 2009a) and used in mate choice (Robertson and Monteiro 2005) in opaque species. Experimental studies are needed to clarify this point.

\section{Ecological relevance of transparency for thermoregulation}

The general patterns of variations in transmittance with latitudinal distance to the equator support the idea that transparency may be involved in thermoregulation. While transmittance shows its largest variation in the tropics, it decreases as at higher latitudes, with an average loss of $4-5 \%$ transmittance per $10^{\circ}$ increase in latitude. Such variations concern the near-infrared (700$1,100 \mathrm{~nm})$ range and the human-visible (400-700 $\mathrm{nm})$ range, but not the UV range $(300-400 \mathrm{~nm})$. Given that transmission + reflection + absorption $=1$, it is reasonable to think that lower direct transmittance values likely translate, at least in part, into higher absorption levels, if reflection values and diffuse transmittance values are roughly maintained at the same levels. In this context, we can interpret the decrease in transmittance with increasing latitude as an increase in absorption with increasing latitude. Our results are in agreement with previous support of the thermal melanism hypothesis (Zeuss et al. 2014, Heidrich et al. 2018, Xing et al. 2018, Stelbrink et al. 2019). Contrary to Munro et al.'s (2019) study in Australian opaque butterflies, where reflectance decreases (absorption increases) with increasing latitude 
more strongly in the infrared range than at shorter wavelengths, we find similar variations in the near infrared and human-visible ranges. Such similarity in the two ranges could be due to several reasons. First, chitin and melanin refraction indexes and absorption coefficients show lower wavelength-dependent variations at long than at short wavelengths (Azofeifa et al. 2012, Stavenga et al. 2014), resulting in more similar properties at longer wavelengths. Second, light intensity levels determine the maximal sighting distance at which a transparent object can be detected (Johnsen and Widder 1998, Ruxton et al. 2004). A poorly transmitting target seen in dimmer light can be as difficult to detect as a highly transmitting target seen in brighter light. Light intensities are higher in the tropics than in the temperate zone (Gueymard and Ruiz-Arias 2016) and visual systems may therefore select for higher transmittance in the tropics. As a consequence, thermoregulation (in the infrared range) and vision (at shorter wavelengths) may act concurrently and contribute to creating an autocorrelation. More studies are needed to further explore the variations in absorption of clearwing Lepidoptera, and to decipher the relative contribution of vision and thermoregulation to variation in transparency at large geographical scale.

Munro et al. (2019) have found that climate correlates to body and proximal wing coloration. Given that we have measured five points at various locations within the transparent zone, and that the transparent zone is not always located close to the insect body, finding a general correlation between latitude and transparency suggests it does not depend on the location of the transparent zone on the wing, and its proximity to the insect body. Whether optical variations exist within transparent zones in relation to the distance to the insect body requires further investigation. Contrary to Munro et al.'s (2019) study, we did not find that latitudinal variations were more pronounced in smaller species.

Ultimately, whether transparent patches are involved in thermoregulation depend not only on intrinsic thermal wing properties, but also on species behavior (basking or seeking shade, flight activity, shivering). Selection can act on both components. Although the potential thermal benefit offered by transparency may seem small compared to that of opaque patches, benefits accumulated over a life-time can be substantial (Stuart-Fox et al. 2017). Studies are needed to explore the relative contribution of physics and behavior to the thermoregulation in clearwing Lepidoptera.

\section{Ecological relevance of transparency for UV protection?}

Transmittance spectra show a constant pattern, whereby UV transmittance is always lower than transmittance at longer wavelengths. This suggests an absorption in the UV range, as shown by chitin and melanin specific absorption curves (Wolbarsht et al. 1981, Azofeifa et al. 2012, Stavenga et al. 2014). This can be the by-product of selection for a transparent material (which inevitably absorbs more at shorter wavelengths) or this could be interpreted as a protection against UV irrespective of latitudinal variations. If transparency plays a role in UV protection (and in protection against pathogens), we should expect lower transmission (a higher absorption with similar reflection levels) at lower latitudes, where UV radiation levels are higher and pathogens more abundant. Instead, our results show that UV transmission in the UV range (in absolute or in proportion) does not vary with latitude, suggesting that thermoregulation rather than protection against UV or pathogens drives transparency features in Lepidoptera. In the literature on opaque coloration, agreement with thermoregulation but not with UV protection has been found in the coloration of bird eggs, for which thermoregulation is crucial to ensure embryo development (Wisocki et al. 2020).

\section{Conclusion}

Transparency has evolved multiple times independently in an insect order characterized by wing opacity. These multiple gains have led to a large diversity of structural strategies that enable transparency. Optical transparency is determined by both macrostructure (length, area, proportion of clearwing area) and microstructure (scale dimensions, type, insertion, coloration, and density). Microstructural traits are tightly linked in their evolution, leading to differential investment in chitin and pigments between structural strategies. Physical transparency translates into visually effective concealment with interesting size-dependent differences. The links between transparency and latitude are consistent with thermal benefits, and much less with UV protection. In many ways, our findings echo the results that have been found in opaque Lepidoptera, showing that transparency is more complex than just enhancing concealment and is likely a multifunctional compromise. Future studies should expand measurement configurations, explore the developmental pathways of transparency, its intraspecific variations, and experimentally test its putative adaptive functions and potential trade-offs with other vital functions such as water repellency or flight ability.

\section{ACKNOWLEDGMENTS}

This work was funded by Clearwing ANR project (ANR-16CE02-0012), HFSP project on transparency (RGP0014/2016) and a France-Berkeley fund grant (FBF \#2015---58). We thank Peruvian and Ecuadorian authorities to allow us conducting fieldwork that inspired this study (authorizations 373-2017SERFOR-DGGSPFFS, 019-IC-FAU-DNBAPVS/MA). We warmly thank Jacques Pierre and Rodolphe Rougerie for help with species choice, identification, and data on species ecology, Edgar Attivissimo for contributing to Keyence imaging, and Thibaud Decaëns, Daniel Herbin, and Claude Tautel for species selection and identification. We thank Axel Hasmann, Ulf Buchsbaum, and Gottfried Behounek, Jayme Sones, Jeremy deWaard, and Franz Pühringer for giving us access to private 
BOLD sequences. We also deeply thank Matthew Forister, Sönke Johnsen, and two anonymous reviewers for their helpful comments on the manuscript. D. Gomez and M. Elias designed the questions, with contributions from N. Patel, C. Andraud, S. Barbut, and A. Pomerantz to the protocol; D. Gomez, M. Elias, J. Barbut, W. Daney de Marcillac, and J. Pairraire performed species selection and optical and structural measurements; D. Gomez, M. Elias, M. Aarias, and C. Pinna performed the analyses; and D. Gomez wrote the manuscript, with contribution from all co-authors.

\section{Literature Cited}

Arias, M., M. Elias, C. Andraud, S. Berthier, and D. Gomez. 2020. Transparency improves concealment in cryptically coloured moths. Journal of Evolutionary Biology 33:247252.

Arias, M., J. Mappes, C. Desbois, S. Gordon, M. McClure, M. Elias, O. Nokelainen, and D. Gomez. 2019. Transparency reduces predator detection in chemically protected clearwing butterflies. Functional Ecology 33:1110-1119.

Azofeifa, D. E., H. J. Arguedas, and W. E. Vargas. 2012. Optical properties of chitin and chitosan biopolymers with application to structural color analysis. Optical Materials 35:175183.

Bagge, L. E. 2019. Not as clear as it may appear: challenges associated with transparent camouflage in the ocean. Integrative and Comparative Biology 59:1653-1663.

Beckmann, M., T. Václavík, A. M. Manceur, L. Šprtová, H. von Wehrden, E. Welk, and A. F. Cord. 2014. glUV: a global UV-B radiation data set for macroecological studies. Methods in Ecology and Evolution 5:372-383.

Berthier, S. 2005. Thermoregulation and spectral selectivity of the tropical butterfly Prepona meander: a remarkable example of temperature auto-regulation. Applied Physics a-Materials Science \& Processing 80:1397-1400.

Berthier, S. 2007. Iridescences: the physical colors of insects. Springer, Berlin, Germany.

Binetti, V. R., J. D. Schiffman, O. D. Leaffer, J. E. Spanier, and C. L. Schauer. 2009. The natural transparency and piezoelectric response of the Greta oto butterfly wing. Integrative Biology 1:324-329.

Blomberg, S. P., T. Garland, and A. R. Ives. 2003. Testing for phylogenetic signal in comparative data: behavioral traits are more labile. Evolution 57:717-745.

Bogert, C. M. 1949. Thermoregulation in reptiles, a factor in evolution. Evolution 3:195-211.

Brakefield, P. M. 1987. Industrial melanism: do we have the answers? Trends in Ecology \& Evolution 2:117-122.

Butler, M., and A. S. Johnson. 2004. Are melanized feather barbs stronger? Journal of Experimental Biology 207:285293.

Bybee, S. M., F. Yuan, M. D. Ramstetter, J. LlorenteBousquets, R. D. Reed, D. Osorio, and A. D. Briscoe. 2012. UV Photoreceptors and UV-yellow wing pigments in heliconius butterflies allow a color signal to serve both mimicry and intraspecific communication. American Naturalist 179:38-51.

Campenhausen, M. V., and K. Kirschfeld. 1998. Spectral sensitivity of the accessory optic system of the pigeon. Journal of Comparative Physiology, Series A 183:1-6.

Costello, L. M., N. E. Scott-Samuel, K. Kjernsmo, and I. C. Cuthill. 2020. False holes as camouflage. Proceedings of the Royal Society B 287:20200126.

Cuthill, I. C. 2019. Camouflage. Journal of Zoology 308:75-92.

Deparis, O., S. Mouchet, L. Dellieu, J.-F. Colomer, and M. Sarrazin. 2014. Nanostructured surfaces: bioinspiration for transparency, coloration and wettability. Materials Today: Proceedings 1:122-129.

Drummond, A. J., and A. Rambaut. 2007. BEAST: Bayesian evolutionary analysis by sampling trees. BMC Evolutionary Biology 7:214.

Drummond, A. J., M. A. Suchard, D. Xie, and A. Rambaut. 2012. Bayesian phylogenetics with BEAUti and the BEAST 1.7. Molecular Biology and Evolution 29:1969-1973.

Elbourne, A., R. J. Crawford, and E. P. Ivanova. 2017. Nanostructured antimicrobial surfaces: from nature to synthetic analogues. Journal of Colloid and Interface Science 508:603616.

Ellers, J., and C. L. Boggs. 2004. Functional ecological implications of intraspecific differences in wing melanization in Colias butterflies. Biological Journal of the Linnean Society 82:79-87.

Endler, J. A. 1993. The color of light in forests and its implications. Ecological Monographs 63:1-27.

Fang, Y., G. Sun, Y. H. Bi, and H. Zhi. 2015. Multipledimensional micro/nano structural models for hydrophobicity of butterfly wing surfaces and coupling mechanism. Science Bulletin 60:256-263.

Farina, J. L. 2001. Aportes al conocimiento de la bionomía de Heliconisa pagenstecheri (Lepidoptera: Saturniidae). Revista de la Sociedad Entomológica Argentina 60:125-128.

Fritz, S. A., and A. Purvis. 2010. Selectivity in mammalian extinction risk and threat types: a new measure of phylogenetic signal strength in binary traits. Conservation Biology 24:1042-1051.

Gomez, D. 2021. ClearwingLepidoptera. Open Science Framework. https://doi.org/10.17605/OSF.IO/Q8TWE

Goodwyn, P. P., E. De Souza, K. Fujisaki, and S. Gorb. 2008. Moulding technique demonstrates the contribution of surface geometry to the super-hydrophobic properties of the surface of a water strider. Acta Biomaterialia 4:766-770.

Goodwyn, P. P., Y. Maezono, N. Hosoda, and K. Fujisaki. 2009. Waterproof and translucent wings at the same time: problems and solutions in butterflies. Naturwissenschaften 96:781-787.

Gueymard, C. A., and J. A. Ruiz-Arias. 2016. Extensive worldwide validation and climate sensitivity analysis of direct irradiance predictions from 1-min global irradiance. Solar Energy 128:1-30.

Guillerme, T., and K. Healy. 2019. mulTree: performs MCMCglmm on multiple phylogenetic trees. $\mathrm{R}$ package version 1.3.6. https://github.com/TGuillerme/mulTree

Hadfield, J. D. 2010. MCMC methods for multi-response generalized linear mixed models: the MCMCglmm $R$ Package. Journal of Statistical Software 33:1-22.

Hart, N. S. 2004. Microspectrophotometry of visual pigments and oil droplets in a marine bird, the wedge-tail shearwater Puffinus pacificus: topographic variation in photoreceptor spectral characteristics. Journal of Experimental Biology 207:1229-1240.

Hart, N. S., J. C. Partridge, I. C. Cuthill, and A. T. D. Bennett. 2000. Visual pigments, oil droplets, ocular media and cone photoreceptor distribution in two species of passerine bird: the blue tit (Parus caeruleus L.) and the blackbird (Turdus merula L.). Journal of Comparative Physiology, Series A 186:375-387.

Hart, N. S., and M. Vorobyev. 2005. Modelling oil droplet absorption spectra and spectral sensitivities of bird cone photoreceptors. Journal of Comparative Physiology, Series A 191:381-392.

Heidrich, L., N. Friess, K. Fiedler, M. Braendle, A. Hausmann, R. Brandl, and D. Zeuss. 2018. The dark side of Lepidoptera: 
colour lightness of geometrid moths decreases with increasing latitude. Global Ecology and Biogeography 27:407-416.

Hernandez-Chavarria, F., A. Hernandez, and A. Sittenfeld. 2004. The "windows" scales, and bristles of the tropical moth Rothschildia lebeau (Lepidoptera: Saturniidae). Revista de Biologia Tropical 52:919-926.

Janssen, J. M., A. Monteiro, and P. M. Brakefield. 2001. Correlations between scale structure and pigmentation in butterfly wings. Evolution \& Development 3:415-423.

Janzen, D. H. 1984. Weather-related color polymorphism of Rothschildia lebeau (Saturniidae). Bulletin of the Entomological Society of America 30:16-21.

Johnsen, S. 2001. Hidden in plain sight: The ecology and physiology of organismal transparency. Biological Bulletin 201:301-318.

Johnsen, S. 2014. Hide and seek in the open sea: pelagic camouflage and visual countermeasures. Annual Review of Marine Science 6:369-392.

Johnsen, S., and E. A. Widder. 1998. Transparency and visibility of gelatinous zooplankton from the Northwestern Atlantic and Gulf of Mexico. Biological Bulletin 195:337-348.

Kang, C., R. Zahiri, and T. N. Sherratt. 2017. Body size affects the evolution of hidden colour signals in moths. Proceedings of the Royal Society B 284:20171287.

Kawahara, A. Y., et al. 2019. Phylogenomics reveals the evolutionary timing and pattern of butterflies and moths. Proceedings of the National Academy of Sciences USA 116:2265722663 .

Kembel, S. W., P. D. Cowan, M. R. Helmus, W. K. Cornwell, H. Morlon, D. D. Ackerly, S. P. Blomberg, and C. O. Webb. 2010. Picante: R tools for integrating phylogenies and ecology. Bioinformatics 26:1463-1464.

Kemp, D. J. 2007. Female butterflies prefer males bearing bright iridescent ornamentation. Proceedings of the Royal Society B 274:1043-1047.

Koshkin, E. S., and S. I. Yevdoshenko. 2019. Diversity and ecology of hawk moths of the genus Hemaris (Lepidoptera, Sphingidae) of the Russian Far East. Journal of Asia-Pacific Biodiversity 12:613-625.

Krishna, A., X. Nie, A. D. Warren, J. E. Llorente-Bousquets, A. D. Briscoe, and J. Lee. 2020. Infrared optical and thermal properties of microstructures in butterfly wings. Proceedings of the National Academy of Sciences USA 117:1566-1572.

Kristensen, N. P., M. Fischer, W. Kükenthal, M. Beier, T. Krumbach, and J. Niethammer, editors. 2003. Lepidoptera, moths and butterflies; 2. Morphology, physiology, and development. de Gruyter, Berlin, Germany.

Lanfear, R., P. B. Frandsen, A. M. Wright, T. Senfeld, and B. Calcott. 2017. PartitionFinder 2: new methods for selecting partitioned models of evolution for molecular and morphologicalphylogenetic analyses. Molecular Biology and Evolution 34:772-773.

Lind, O., S. Karlsson, and A. Kelber. 2013. Brightness discrimination in budgerigars (Melopsittacus undulatus). PLoS ONE 8:e54650.

Lind, O., M. Mitkus, P. Olsson, and A. Kelber. 2014. Ultraviolet vision in birds: the importance of transparent eye media. Proceedings of the Royal Society B 281:20132209.

Liu, Y., Y. Song, S. Niu, Y. Zhang, Z. Han, and L. Ren. 2016. Integrated super-hydrophobic and antireflective PDMS biotemplated from nano-conical structures of cicada wings. RSC Advances 6:108974-108980.

Maia, R., H. Gruson, J. A. Endler, and T. E. White. 2019. pavo 2: New tools for the spectral and spatial analysis of colour in R. Methods in Ecology and Evolution 10:1097-1107.

Maier, E. J., and J. K. Bowmaker. 1993. Colour vision in the passeriform bird, Leiothrix lutea: correlation of visual pigment absorbance and oil droplet transmission with spectral sensitivity. Journal of Comparative Physiology, Series A 172:295-301.

Matsuoka, Y., and A. Monteiro. 2018. Melanin pathway genes regulate color and morphology of butterfly wing scales. Cell Reports 24:56-65.

McClure, M., et al. 2019. Why has transparency evolved in aposematic butterflies? Insights from the largest radiation of aposematic butterflies, the Ithomiini. Proceedings of the Royal Society B 286:20182769.

Miaoulis, I. N., and B. D. Heilman. 1998. Butterfly thin films serve as solar collectors. Annals of the Entomological Society of America 91:122-127.

Miller, M. A., W. Pfeiffer, and T. Schwartz. 2010. Creating the CIPRES Science Gateway for inference of large phylogenetic trees. Pages 1-8 in 2010 Gateway Computing Environments Workshop (GCE). IEEE.

Mueller, K. P., and S. C. F. Neuhauss. 2014. Sunscreen for fish: co-option of UV light protection for camouflage. PLoS ONE 9:e87372.

Munro, J. T., I. Medina, K. Walker, A. Moussalli, M. R. Kearney, A. G. Dyer, J. Garcia, K. J. Rankin, and D. Stuart-Fox. 2019. Climate is a strong predictor of near-infrared reflectance but a poor predictor of colour in butterflies. Proceedings of the Royal Society B 286:20190234.

Nosonovsky, M., and B. Bhushan. 2007. Hierarchical roughness makes superhydrophobic states stable. Microelectronic Engineering 84:382-386.

Odeen, A., O. Hastad, and P. Alstrom. 2011. Evolution of ultraviolet vision in the largest avian radiation - the passerines. BMC Evolutionary Biology 11:313.

Orme, C. D. L., R. P. Freckleton, G. H. Thomas, T. Petzoldt, S. A. Fritz, N. J. B. Isaac, and W. Pearse. 2018. Caper: comparative analyses of phylogenetics and evolution in R. https:// cran.r-project.org/web/packages/caper/

Pagel, M. 1999. Inferring the historical patterns of biological evolution. Nature 401:877-884.

Pinheiro, J., D. Bates, D. DebRoy, and D. R. Sarkar. 2020. nlme: linear and nonlinear mixed effects models. $\mathrm{R}$ package version 3.1-145. https://cran.r-project.org/web/packages/nlme/

Pinna, C., M. Vilbert, S. Borenztajn, W. D. de Marcillac, F. Piron-Prunier, A. Pomerantz, N. Patel, S. Berthier, C. Andraud, D. Gomez, and M. Elias. 2020. Convergence in light transmission properties of transparent wing areas in clearwing mimetic butterflies. bioRxiv:2020.06.30.180612.

Pomerantz, A. F., R. H. Siddique, E. I. Cash, Y. Kishi, C. Pinna, K. Hammar, D. Gomez, M. Elias, and N. H. Patel. 2021. Developmental, cellular, and biochemical basis of transparency in clearwing butterflies. Journal of Experimental Biology 224:jeb237917.

R Development Core Team. 2013. R: a language and environment for statistical computing. R Foundation for Statistical Computing, Vienna, Austria. www.R-project.org

Ratnasingham, S., and P. D. N. Hebert. 2007. BOLD: the barcode of life data system (http://www.barcodinglife.org). Molecular Ecology Notes 7:355-364.

Revell, L. J. 2012. phytools: An R package for phylogenetic comparative biology (and other things). Methods in Ecology and Evolution 3:217-223.

Robertson, K. A., and A. Monteiro. 2005. Female Bicyclus anynana butterflies choose males on the basis of their dorsal UVreflective eyespot pupils. Proceedings of the Royal Society B 272:1541-1546.

Ruxton, G. D., T. N. Sherratt, and M. P. Speed. 2004. Avoiding attack - the evolutionary ecology of crypsis, warning signals, and mimicry. Oxford University Press, New York, New York, USA. 
Schmidt, B. C. 2018. Cryptic species among bumblebee mimics: an unrecognized Hemaris hawkmoth (Lepidoptera: Sphingidae) in eastern North America. Zootaxa 4399:32-48.

Schneider, C. A., W. S. Rasband, and K. W. Eliceiri. 2012. NIH Image to ImageJ: 25 years of image analysis. Nature Methods 9:671-675.

Siddique, R. H., G. Gomard, and H. Holscher. 2015. The role of random nanostructures for the omnidirectional antireflection properties of the glasswing butterfly. Nature Communications 6:6909.

Smith, S. A., and C. W. Dunn. 2008. Phyutility: a phyloinformatics tool for trees, alignments and molecular data. Bioinformatics 24:715-716.

Stavenga, D. G., H. L. Leertouwer, and B. D. Wilts. 2014. The colouration toolkit of the pipevine swallowtail butterfly, Battus philenor: thin films, papiliochromes, and melanin. Journal of Comparative Physiology A 200:547-561.

Stavenga, D. G., A. Matsushita, K. Arikawa, H. L. Leertouwer, and B. D. Wilts. 2012. Glass scales on the wing of the swordtail butterfly Graphium sarpedon act as thin film polarizing reflectors. Journal of Experimental Biology 215:657-662.

Stelbrink, P., S. Pinkert, S. Brunzel, J. Kerr, C. W. Wheat, R. Brandl, and D. Zeuss. 2019. Colour lightness of butterfly assemblages across North America and Europe. Scientific Reports 9:1760.

Stevens, M., A. Cantor, J. Graham, and I. S. Winney. $2009 a$ The function of animal "eyespots": conspicuousness but not eye mimicry is key. Current Zoology 55:319-326.

Stevens, M., I. S. Winney, A. Cantor, and J. Graham. $2009 b$. Outline and surface disruption in animal camouflage. Proceedings of the Royal Society B 276:781-786.

Stevens, M., C. L. Stubbins, and C. J. Hardman. 2008. The antipredator function of "eyespots" on camouflaged and conspicuous prey. Behavioral Ecology and Sociobiology 62:1787-1793.

Stoffel, M. A., S. Nakagawa, and H. Schielzeth. 2017. rptR: repeatability estimation and variance decomposition by generalized linear mixed-effects models. Methods in Ecology and Evolution 8:1639-1644.

Stuart-Fox, D., E. Newton, and S. Clusella-Trullas. 2017. Thermal consequences of colour and near-infrared reflectance. Philosophical Transactions of the Royal Society B 372:20160345.

Sugumaran, M. 2009. Complexities of cuticular pigmentation in insects. Pigment Cell \& Melanoma Research 22:523-525.

True, J. R. 2003. Insect melanism: the molecules matter. Trends in Ecology \& Evolution 18:640-647.
Tuthill, J. C., and S. Johnsen. 2006. Polarization sensitivity in the red swamp crayfish Procambarus clarkii enhances the detection of moving transparent objects. Journal of Experimental Biology 209:1612-1616.

Vorobyev, M., and D. Osorio. 1998. Receptor noise as a determinant of colour thresholds. Proceedings of the Royal Society B 265:351-358.

Wagner, T., C. Neinhuis, and W. Barthlott. 1996. Wettability and contaminability of insect wings as a function of their surface sculptures. Acta Zoologica 77:213-225.

Wanasekara, N. D., and V. B. Chalivendra. 2011. Role of surface roughness on wettability and coefficient of restitution in butterfly wings. Soft Matter 7:373-379.

Webb, C. O. 2000. Exploring the phylogenetic structure of ecological communities: an example for rain forest trees. American Naturalist 156:145-155.

Wisocki, P. A., P. Kennelly, I. R. Rivera, P. Cassey, M. L. Burkey, and D. Hanley. 2020. The global distribution of avian eggshell colours suggest a thermoregulatory benefit of darker pigmentation. Nature Ecology \& Evolution 4:148-155.

Wolbarsht, M. L., A. W. Walsh, and G. George. 1981. Melanin, a unique biological absorber. Applied Optics 20:2184-2186.

Xing, S., T. C. Bonebrake, L. A. Ashton, R. L. Kitching, M. Cao, Z. Sun, J. C. Ho, and A. Nakamura. 2018. Colors of night: climate-morphology relationships of geometrid moths along spatial gradients in southwestern China. Oecologia 188:537-546.

Yoshida, A., M. Motoyama, A. Kosaku, and K. Miyamoto. 1996. Nanoprotuberance array in the transparent wing of a hawkmoth, Cephonodes hylas. Zoological Science 13:525-526.

Yoshida, A., M. Motoyama, A. Kosaku, and K. Miyamoto. 1997. Antireflective nanoprotuberance array in the transparent wing of a hawkmoth, Cephonodes hylas. Zoological Science 14:737-741

Zeuss, D., R. Brandl, M. Brändle, C. Rahbek, and S. Brunzel. 2014. Global warming favours light-coloured insects in Europe. Nature Communications 5:3874.

Zhang, C.-Y., J.-Y. Meng, X.-P. Wang, F. Zhu, and C.-L. Lei. 2011. Effects of UV-A exposures on longevity and reproduction in Helicoverpa armigera, and on the development of its F1 generation. Insect Science 18:697-702.

Zheng, Y. M., X. F. Gao, and L. Jiang. 2007. Directional adhesion of superhydrophobic butterfly wings. Soft Matter 3:178182.

Zikán, J. F. 1927. Biologie der Heliconisa-Arten (Lep. Saturnid.). Berliner Entomologische Zeitschrift 1927:58-82.

\section{SUPPORTING INFORMATION}

Additional supporting information may be found online at: http://onlinelibrary.wiley.com/doi/10.1002/ecm.1475/full

$$
\text { OPEN RESEARCH }
$$

Data (Gomez 2021) are available on the Open Science Framework at: https://doi.org/10.17605/OSF.IO/Q8TWE. 
Supporting information. D. Gomez, C. Pinna, J. Pairraire, M. Arias, J. Barbut, A. Pomerantz, W. Daney de Marcillac, S. Berthier, N. Patel, C. Andraud, M. Elias. 2020. Wing transparency in butterflies and moths: structural diversity, optical properties and ecological relevance. Ecological Monographs.

Table S1. Sample sizes for each structural category.

\begin{tabular}{|c|c|c|c|c|c|c|c|c|c|}
\hline & \multicolumn{3}{|c|}{ type+insertion+colouration } & \multicolumn{3}{|c|}{ type+insertion } & \multicolumn{3}{|c|}{ type } \\
\hline & & $\begin{array}{l}\text { Species } \\
\text { number }\end{array}$ & $\begin{array}{l}\text { Family } \\
\text { number }\end{array}$ & & $\begin{array}{l}\text { Species } \\
\text { number }\end{array}$ & $\begin{array}{l}\text { Family } \\
\text { number }\end{array}$ & & $\begin{array}{l}\text { Species } \\
\text { number }\end{array}$ & $\begin{array}{l}\text { Family } \\
\text { number }\end{array}$ \\
\hline \multirow{11}{*}{$\begin{array}{l}\text { Transparent } \\
\text { zone }\end{array}$} & PEC & 15 & 5 & PE & 15 & 5 & $\mathrm{D}$ & 27 & a \\
\hline & PFC & 12 & 6 & $\mathrm{PF}$ & 12 & 6 & $r$ & 21 & $y$ \\
\hline & PLEC & 4 & 2 & PIF & 5 & 3 & \multirow{4}{*}{$\mathrm{PL}$} & \multirow{4}{*}{12} & \multirow{4}{*}{7} \\
\hline & PLET & 1 & 1 & \multirow{3}{*}{ PLF } & 5 & 3 & & & \\
\hline & PLFC & 3 & 3 & & \multirow{2}{*}{7} & \multirow{2}{*}{6} & & & \\
\hline & PLFT & 4 & 4 & & & & & & \\
\hline & LEC & 16 & 8 & IF & 25 & 13 & \multirow{4}{*}{$\mathrm{L}$} & \multirow{4}{*}{69} & \multirow{4}{*}{26} \\
\hline & LET & 9 & 6 & LL & \multirow{3}{*}{48} & \multirow{3}{*}{21} & & & \\
\hline & LFC & 27 & 13 & \multirow{2}{*}{$\mathrm{LF}$} & & & & & \\
\hline & LFT & 22 & 13 & & & & & & \\
\hline & $\mathrm{N}^{*}$ & $\begin{array}{c}33 \\
\text { (22 nude, } 11 \text { with sockets) }\end{array}$ & $\begin{array}{c}12 \\
\text { (8 nude, } 6 \text { with sockets) }\end{array}$ & $\mathrm{N}^{*}$ & 33 & 12 & $\mathrm{~N}^{*}$ & 33 & 12 \\
\hline \multirow{4}{*}{$\begin{array}{l}\text { Opaque } \\
\text { zone }\end{array}$} & PFC & 5 & 5 & PF & 5 & 5 & $\mathrm{P}$ & 5 & 5 \\
\hline & PLFC & 1 & 1 & PLF & 1 & 1 & $\mathrm{PL}$ & 1 & 1 \\
\hline & LEC & 1 & 1 & LE & 1 & 1 & \multirow{2}{*}{$\mathrm{L}$} & \multirow{2}{*}{117} & \multirow{2}{*}{30} \\
\hline & LFC & 116 & 30 & $\mathrm{LF}$ & 116 & 30 & & & \\
\hline
\end{tabular}

Type is $\mathrm{P}=$ piliform scales, $\mathrm{L}=$ lamellar scales, $\mathrm{PL}=$ piliform scales and lamellar scales, insertion is. $\mathrm{E}=$ erect, $\mathrm{F}=$ flat and colouration is $\mathrm{C}=$ coloured, $\mathrm{T}=$ transparent. The $\mathrm{N}$ strategy has no scales, no insertion and no colouration. Species can belong to 1 or 2 different strategies depending on whether they had similar or different strategies on their forewing and hindwing We investigated a total of 123 species and 31 families. * within the $\mathrm{N}$ strategy, 25 species and 10 families had a nude membrane only, while 7 species and 4 families had a nude membrane with presence of scale sockets. 
Figure S1. Distribution of structural strategies for transparency across families in the study dataset, in proportion of the total number of species and wings (left), or in absolute number of species (right), with the count of species and families showing a specific strategy. A structural strategy is defined as the combination of scale type, insertion and colouration. Type is $P=$ piliform scales, $L=$ lamellar scales (piliform scales and lamellar scales were assimilated to lamellar scales), insertion is $\mathrm{E}=$ erect, $\mathrm{F}=$ flat and colouration is $\mathrm{C}=$ coloured, $\mathrm{T}=$ transparent. The $\mathrm{N}$ strategy has no scales, no insertion and no colouration. Species that had different strategies on their forewing and hindwing were counted for 0.5 for each strategy. Colours with symbols for proportions are in the same order, but without symbols for species number.
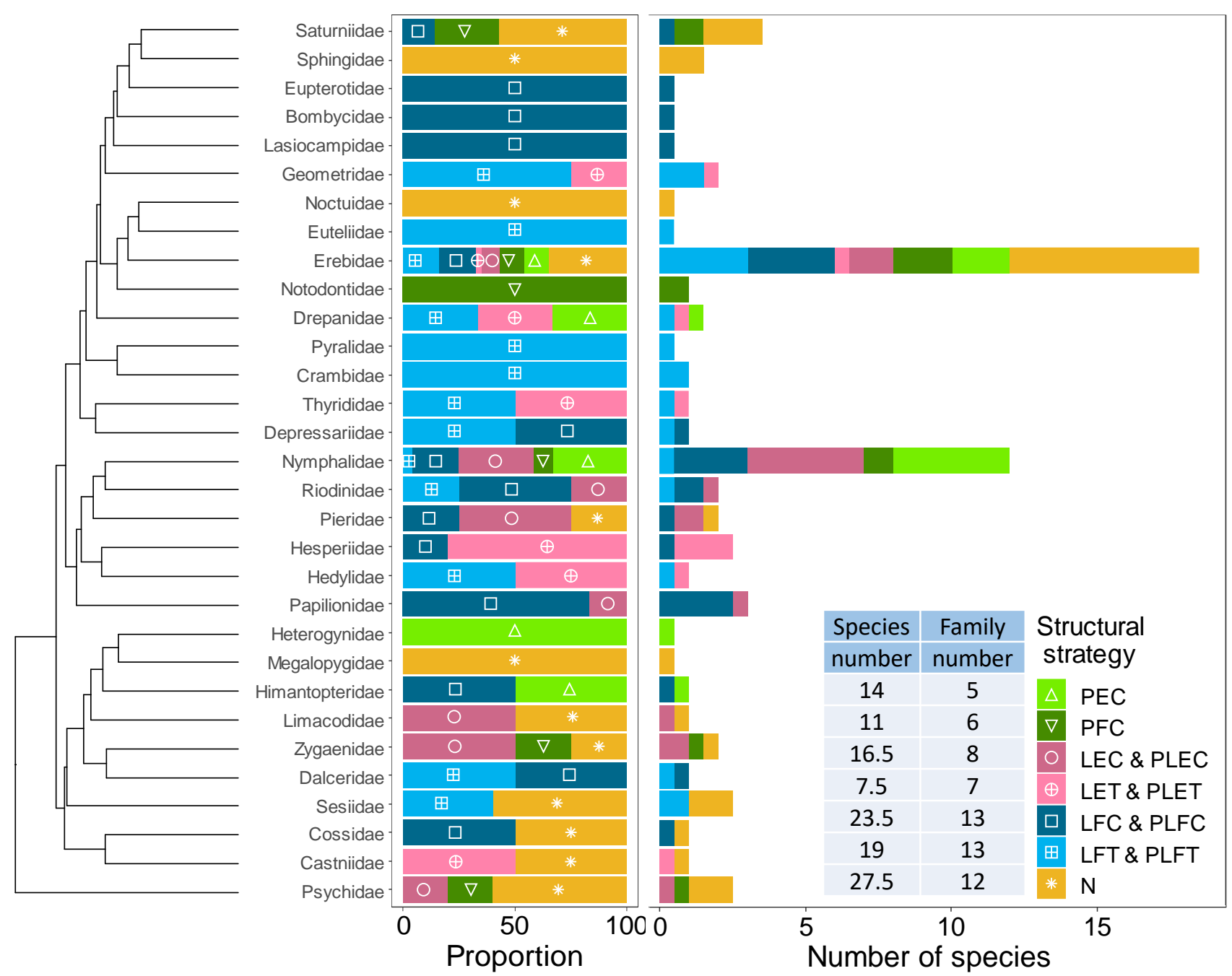
Figure S2. Distribution of some wing structural and species ecological traits. Wing length, proportion of clearwing area, and scale density for the forewing (columns 1, 2, 3) and absolute latitude (column 4). Latitude data (identical to the distance to the equator in degrees) were available only for 77 out of 123 species. Missing data or data not applicable (4 species have no transparent zone for the forewing, hence no scale density) are in white.

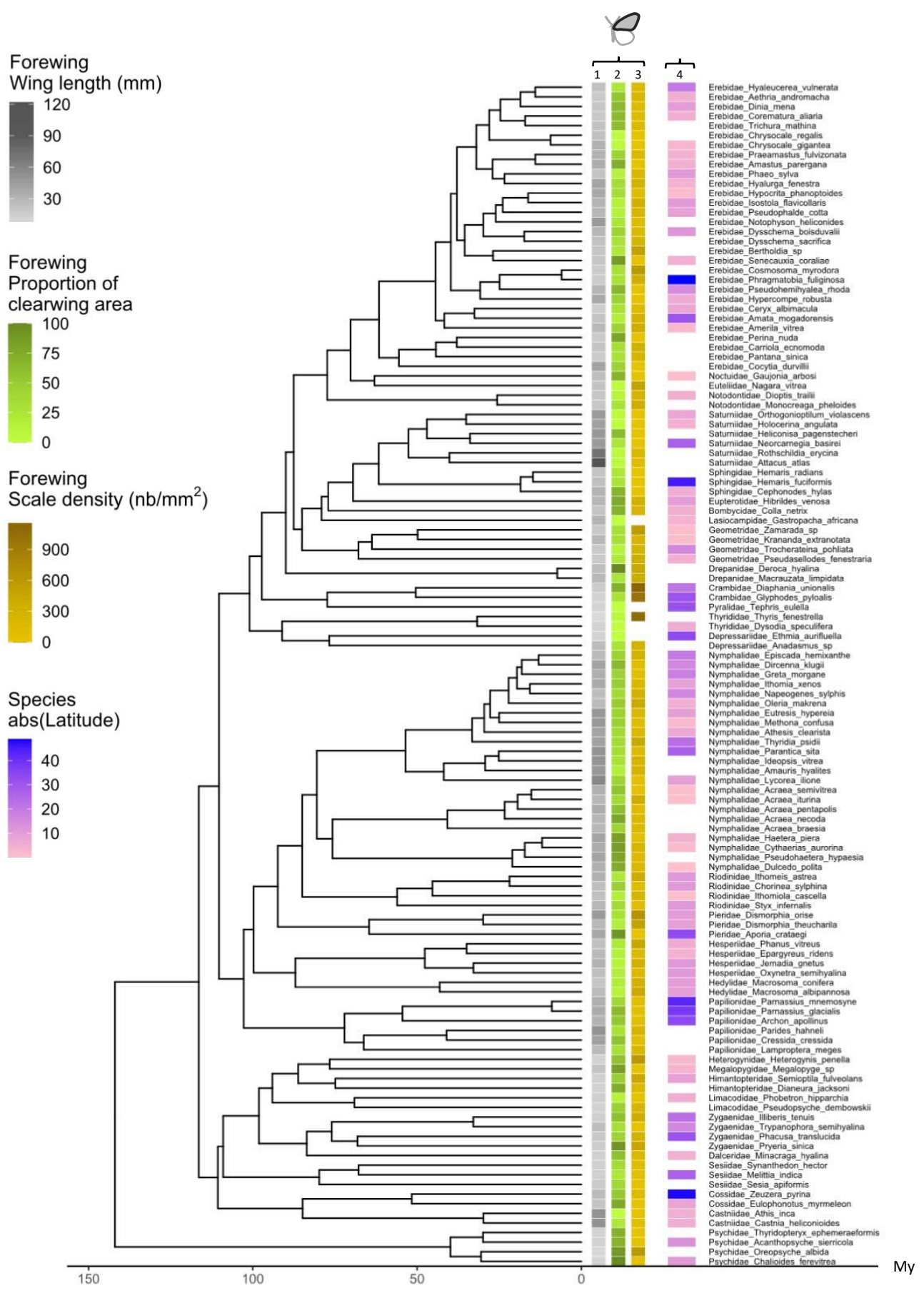


Table S2. Repeatability of variables computed from colour and structure measurements.

\begin{tabular}{|c|c|c|c|}
\hline Variable & $\mathrm{Nb}$ species/measures & Level & $\mathrm{R}( \pm \mathrm{se})$ \\
\hline Mean transmittance over [300-400] & $123 / 1200$ & SpWg & $0.910( \pm 0.009) * * *$ \\
\hline Mean transmittance over [400-700] & $123 / 1200$ & SpWg & $0.911( \pm 0.008) * * *$ \\
\hline Mean transmittance over [700-1100] & $123 / 1200$ & SpWg & $0.893( \pm 0.010) * * *$ \\
\hline Mean transmittance & $123 / 1200$ & SpWg & $0.901( \pm 0.009) * * *$ \\
\hline Proportion of UV transmittance & $123 / 1200$ & $\mathrm{SpWg}$ & $0.884( \pm 0.011) * * *$ \\
\hline Colour contrast ${ }^{\#}$ & $123 / 1200$ & SpWg & $>0.945 * * *$ \\
\hline Brightness contrast ${ }^{\#}$ & $123 / 1200$ & SpWg & $>0.898 * * *$ \\
\hline Wing length & $123 / 492$ & SpWg & $0.999( \pm<0.01) * * *$ \\
\hline Scale density & $30 / 60$ & SpWgZoSca & $0.974( \pm 0.010) * * *$ \\
\hline Scale width & $51 / 168$ & SpWgZoSca & $0.873( \pm 0.027) * * *$ \\
\hline Scale length & $51 / 168$ & SpWgZoSca & $0.651( \pm 0.063) * * *$ \\
\hline Scale surface & $51 / 168$ & SpWgZoSca & $0.899( \pm 0.021) * * *$ \\
\hline
\end{tabular}

For each variable, we specify the number of species measured and the total number of measurements included in the repeatability analysis. For each analysis the level of relevance for the measurement depends on the variable. For colour and wing length, measurements relate to a specific wing $\mathrm{SpWg}$ ), while for scale density and dimensions, measurements relate to a specific wing, zone, scale (SpWgZoSca) defined by its shape, insertion and colouration. If not specified otherwise, mean transmittance was measured across the 300-700 $\mathrm{nm}$ range. We specify the value of repeatability $R$ and the standard error associated se, as well as the significance level ( ${ }^{* *}$ stands for $\mathrm{p}$-value $\left.<0.001\right)$. \# We computed the repeatability for the colour contrast and the brightness contrast for 18 cases: two bird visual systems (UVS and VS), three illuminants (woodland shade, forest shade, large gap), and three backgrounds (sky, leaf, trunk). Results were similar in all cases $(18 / 18)$ and the minimal value of $R$ is indicated. 


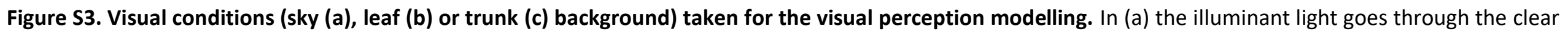

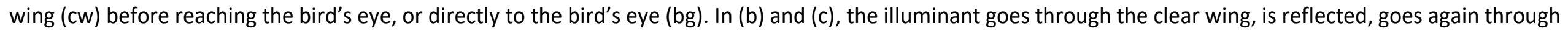

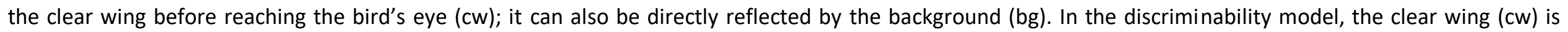
contrasted against the background (bg), under three different illuminants (woodland shade, forest shade, large gap) and by a UVS bird or a VS bird.

a sky background
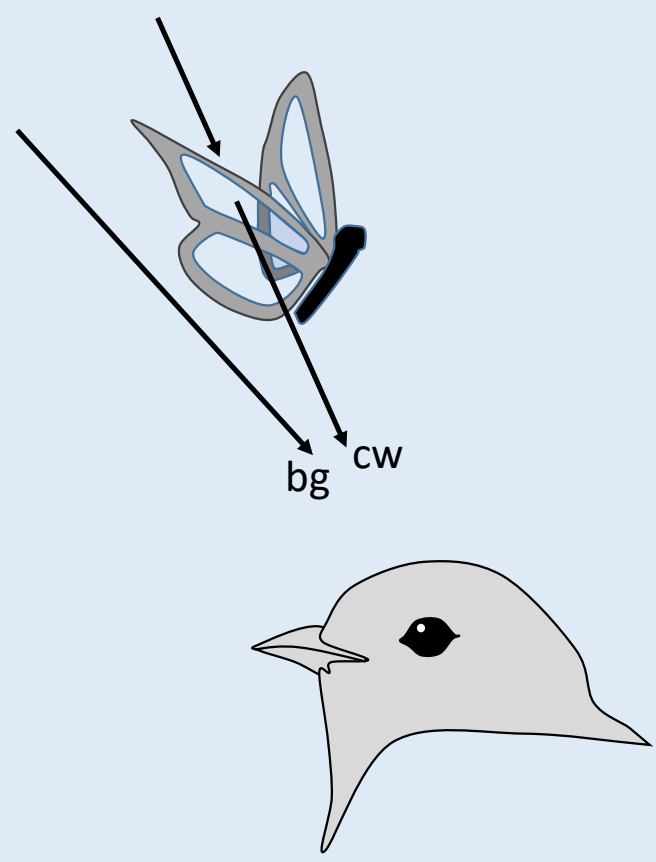

b leaf background

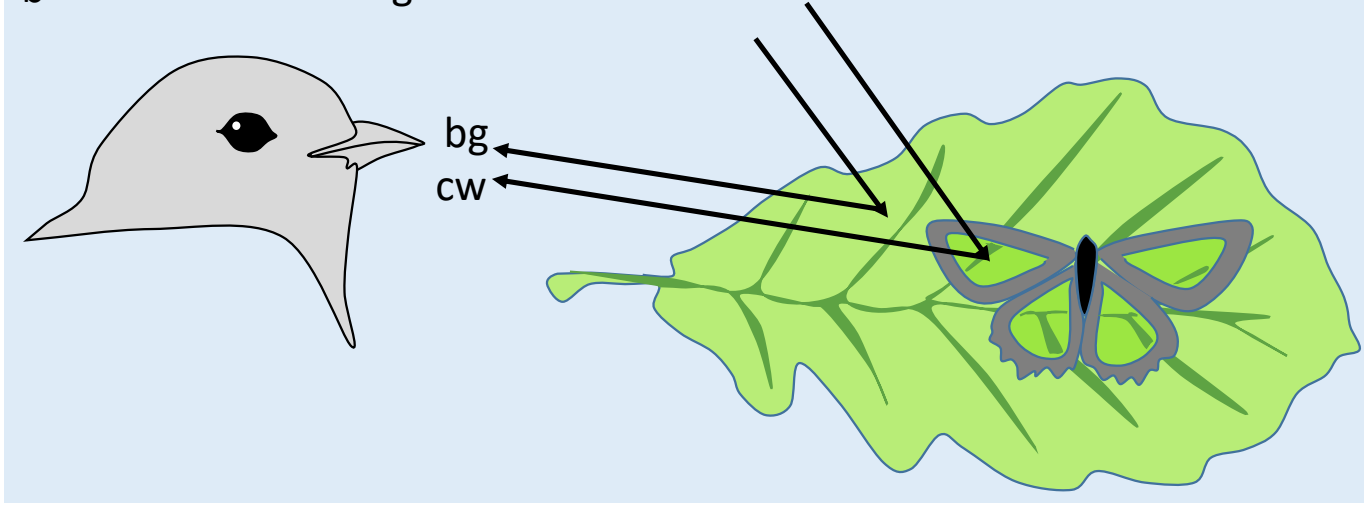

c trunk background

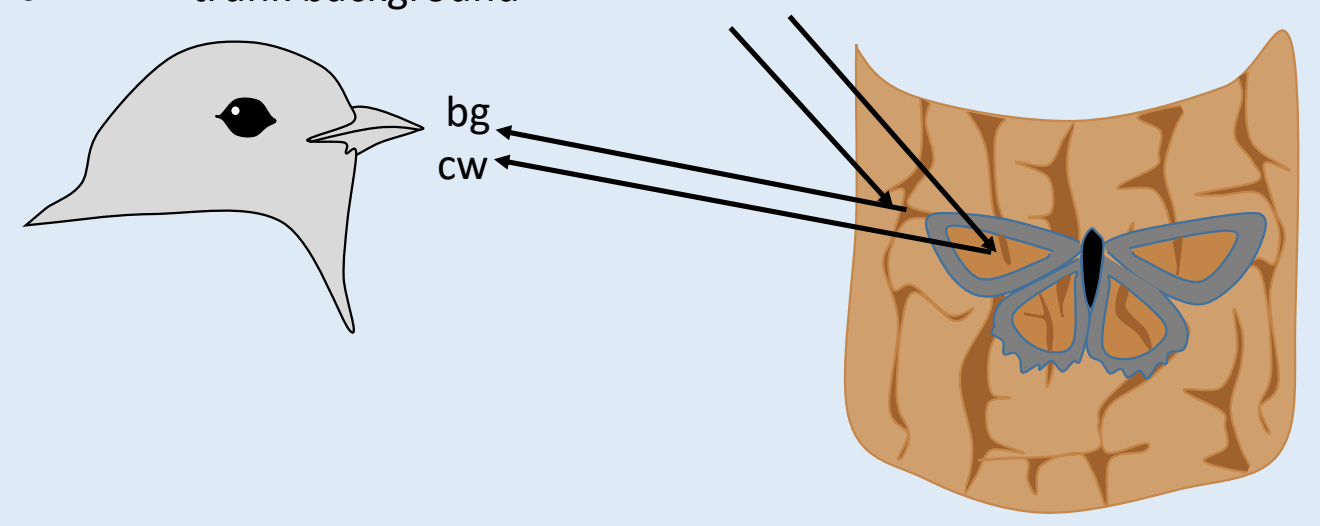


Table S3. Phylogenetic signal on continuous structural and colour variables.

\begin{tabular}{|c|c|c|c|c|c|}
\hline \multirow[b]{3}{*}{ Wing zone } & \multirow[b]{3}{*}{ Variable } & \multirow{2}{*}{\multicolumn{2}{|c|}{ Forewing }} & \multirow{2}{*}{\multicolumn{2}{|c|}{ Hindwing }} \\
\hline & & & & & \\
\hline & & $\mathrm{K}$ & $\lambda$ & K & $\lambda$ \\
\hline \multirow{4}{*}{ Entire wing } & Wing area & $0.682 * *$ & $1.044 * * *$ & $0.668 *$ & $1.043 * * *$ \\
\hline & Clearwing area & $0.652 * * *$ & $0.719 * * *$ & $0.603 * * *$ & $0.697 * * *$ \\
\hline & Proportion of clearwing area & $0.498 * * *$ & $0.671 * * *$ & $0.526 * * *$ & $0.681 * * *$ \\
\hline & Wing length & $1.069 * * *$ & $1.045^{* * *}$ & $0.94 * * *$ & $1.032 * * *$ \\
\hline \multirow{7}{*}{ Transparent } & Mean transmittance over [300-400] & 0.345 & 0 & 0.369 & $0.379 *$ \\
\hline & Mean transmittance over [400-700] & 0.354 & 0 & $0.407 *$ & $0.605^{* *}$ \\
\hline & Mean transmittance over [700-1100] & 0.341 & 0 & $0.402 *$ & $0.606 * * *$ \\
\hline & Mean transmittance over [300-700] & 0.345 & 0 & $0.387 *$ & $0.556 * * *$ \\
\hline & Proportion of UV transmittance & 0.364 & 0 & 0.37 & 0 \\
\hline & Colour contrast ${ }^{\#}$ & $<0.370$ & 0 & $<0.374$ & 0 \\
\hline & Brightness contrast $^{\#}$ & $<0.307$ & $<0.353$ & $>0.544^{*}$ & $>0.878 * * *$ \\
\hline \multirow{4}{*}{ Opaque } & Scale density & $0.469 * *$ & $0.628 * *$ & $0.424 *$ & $0.595^{* *}$ \\
\hline & Scale width & $0.544^{* *}$ & $0.844 * * *$ & $0.607 * * *$ & $0.815^{* * *}$ \\
\hline & Scale length & $0.601 * * *$ & $0.828 * * *$ & 0.421 & 0.179 \\
\hline & Scale surface & $0.696 * * *$ & $0.948 * * *$ & $0.75^{* * *}$ & $0.936 * * *$ \\
\hline \multirow{4}{*}{ Transparent } & Scale density & $0.594 * * *$ & $0.921 * * *$ & $0.656 * * *$ & $0.962 * * *$ \\
\hline & Scale width & $0.49 * *$ & $0.76 * * *$ & $0.639 * *$ & $0.935 * * *$ \\
\hline & Scale length & $0.564 * *$ & $1.03^{* * *}$ & $0.51 * *$ & $0.602 * * *$ \\
\hline & Scale surface & $0.47^{*}$ & $0.671 * * *$ & $0.601 * *$ & $0.938 * * *$ \\
\hline
\end{tabular}

Blomberg's K (Blomberg et al. 2003) and Pagel's $\lambda$ (Pagel 1999) computed for continuous variables. $K$ and $\lambda$ were tested if significantly different from 0 (no phylogenetic signal). Bold values are significant $\mathrm{p}$-values: * $p<0.05, * * p<0.01, * * * p<0.001$. Lambda different from 0 indicates an influence of phylogeny. \# We computed the colour contrast and the brightness contrast for 18 cases: two bird visual systems (UVS and VS), three illuminants (woodland shade, forest shade, large gap), and three backgrounds (sky, leaf, trunk). Results were similar in all cases $(18 / 18)$ for $K$ and $\lambda$, for the forewing and the hindwing. We thus present the threshold values (maximum for $\mathrm{ns}$, minimum for significant values). 
Table S4. Phylogenetic signal on binary structural variables.

\begin{tabular}{|c|c|c|c|c|c|c|c|}
\hline & & \multirow{2}{*}{\multicolumn{3}{|c|}{ Forewing }} & & & \\
\hline & & & & & \multicolumn{3}{|c|}{ Hindwing } \\
\hline Zone & Variable & $D$ & $\begin{array}{c}\mathrm{p}(\mathrm{D}<0) \\
\mathrm{BM}\end{array}$ & $\begin{array}{l}\mathrm{p}(\mathrm{D}>1) \\
\text { random }\end{array}$ & D & $\begin{array}{c}\mathrm{p}(\mathrm{D}<0) \\
\mathrm{BM}\end{array}$ & $\begin{array}{l}\mathrm{p}(\mathrm{D}>1) \\
\text { random }\end{array}$ \\
\hline \multirow{4}{*}{ Transparent } & Presence of scales & 0.137 & $* * *$ & ns & -0.101 & $* * *$ & ns \\
\hline & Scale type & -0.091 & $* * *$ & ns & -0.126 & $* * *$ & ns \\
\hline & Scale insertion & 0.487 & $* *$ & ns & 0.528 & $* *$ & $*$ \\
\hline & Scale colouration & -0.013 & $* * *$ & ns & 0.107 & $* * *$ & ns \\
\hline \multirow{2}{*}{ Opaque } & Scale type & 0.447 & ns & ns & 0.538 & ns & ns \\
\hline & Scale insertion & 14.604 & ns & $* *$ & 11.642 & ns & $* *$ \\
\hline
\end{tabular}

Fritz and Purvis' D (Fritz and Purvis 2010) was computed for the presence of scales (wing membrane covered with scales (whatever their type) or nude), the scale type (piliform scales or lamellar scales, the category piliform scales + lamellar scales being assimilated to lamellar scales), scale insertion on wing membrane ( erect or flat) and scale colouration (containing transparent scales or not). We tested whether D was significantly greater than 0 (departing from Brownian motion) and lower than 1 (departing from random process) and mentioned the associated $p$-value levels: $n s=$ non-significant, ${ }^{*} p<0.05,{ }^{* *} p<0.01,{ }^{* * *} p<0.001$. 
Table S5. Mean-Pairwise Distance (MPD) for binary structure variables

\begin{tabular}{|c|c|c|c|c|c|c|c|}
\hline \multirow{3}{*}{ Zone } & \multirow[b]{3}{*}{ Variable } & \multirow{2}{*}{\multicolumn{2}{|c|}{ Forewing }} & \multirow{2}{*}{\multicolumn{2}{|c|}{ Hindwing }} & \multirow{2}{*}{\multicolumn{2}{|c|}{ At least on one wing }} \\
\hline & & & & & & & \\
\hline & & $\begin{array}{l}\text { Species } \\
\text { number }\end{array}$ & $\begin{array}{c}\text { Mean } \\
\text { age }\end{array}$ & $\begin{array}{l}\text { Species } \\
\text { number }\end{array}$ & $\begin{array}{l}\text { Mean } \\
\text { age }\end{array}$ & $\begin{array}{l}\text { Species } \\
\text { number }\end{array}$ & $\begin{array}{c}\text { Mean } \\
\text { age }\end{array}$ \\
\hline \multirow{19}{*}{ Transparent } & No scales $\mathrm{N}$ & 26 & 103.4 & 29 & $94.5 *$ & 33 & 99.1 \\
\hline & Scales present & 93 & $101 *$ & 90 & 102.6 & 100 & 102.2 \\
\hline & Scales P & 27 & $96.4^{*}$ & 23 & 98.9 & 27 & $96.4^{*}$ \\
\hline & Scales PL & 4 & $63.9 * *$ & 12 & 96.4 & 12 & 96.4 \\
\hline & Scales L & 62 & 102.2 & 55 & 103.9 & 69 & 103.7 \\
\hline & Insertion $\mathrm{E}$ & 42 & $98.1^{*}$ & 34 & 100.6 & 44 & 100.5 \\
\hline & Insertion F & 51 & 100.7 & 56 & 101 & 61 & 100.7 \\
\hline & Colour C & 65 & $99 *$ & 65 & 102.3 & 72 & 101.3 \\
\hline & Colour T & 54 & 103.8 & 54 & 97.8* & 61 & 101.1 \\
\hline & PEC & 15 & $89.5 *$ & 13 & $87.1 *$ & 15 & $89.5 *$ \\
\hline & PFC & 12 & 98 & 10 & 103.9 & 12 & 98 \\
\hline & LEC & 15 & 99.6 & 11 & 107.8 & 16 & 104.9 \\
\hline & LET & 8 & 95.2 & 5 & 105.6 & 9 & 97.9 \\
\hline & LFC & 20 & 99.79 & 24 & 102.5 & 27 & 101.3 \\
\hline & LFT & 19 & 99.7 & 15 & $93 *$ & 22 & 98.9 \\
\hline & PLEC & 3 & $28.1 * *$ & 4 & $56.5^{* *}$ & 4 & $56.5^{* *}$ \\
\hline & PLET & 1 & - & 1 & - & 1 & - \\
\hline & PLFC & 0 & - & 3 & 106.8 & 3 & 106.8 \\
\hline & PLFT & 0 & - & 4 & 99.3 & 4 & 99.3 \\
\hline \multirow{7}{*}{ Opaque } & Scales P & 5 & 120 & 6 & 118.7 & 6 & 118.7 \\
\hline & Scales PL & 1 & - & 12 & $90.1 *$ & 12 & $90.1 *$ \\
\hline & Scales L & 117 & $101.8 * *$ & 105 & 103.1 & 117 & $101.8 * *$ \\
\hline & Insertion $\mathrm{F}$ & 122 & 103.1 & 122 & 103.1 & 122 & 103.1 \\
\hline & LEC & 1 & - & 1 & - & 1 & - \\
\hline & LFC & 116 & $101.8 * *$ & 104 & 103.2 & 116 & $101.8 *$ \\
\hline & PLFC & 1 & - & 12 & $90.1 *$ & 12 & $90.1^{*}$ \\
\hline
\end{tabular}

For a given variable, we considered all the species which had the value indicated for the variable in the table on the forewing, on the hindwing, or on at least one of the wings. We computed the observed MPD, the distribution of 1000 simulated values by randomly shuffling the trait on the phylogeny, and its $5 \%$ inferior quantile threshold. An observed MPD lower than the threshold indicated a significant phylogenetic clustering (indicated by a bold value, corresponding to $p$-value $<0.05$ ). Bold values are significant $p$-values: ${ }^{*} p<0.05$, ${ }^{* *} p<0.01, * * * p<0.001$. Pooling both wings dilutes potential clustering compared to wings taken separately. Instead of expressing MPD values as the length of the branches separating two species, we presented mean ages as MPD/2 to express age depth. A trait shared by all species yielded an MPD-age value of $141.9 \mathrm{My}$, the root age. $\mathrm{P}=$ piliform scales, $\mathrm{L}=$ lamellar scales, $\mathrm{PS}=$ piliform scales and lamellar scales, $\mathrm{F}=$ flat, $\mathrm{E}=$ erect, $\mathrm{T}=$ transparent, $\mathrm{C}=$ coloured. Pooling both wings dilutes potential clustering if any, that is why significant values are a subset of significant values from wings taken separately. The number of species included in the analysis was 119 for the transparent zone of the forewing or the hindwing, 123 otherwise. The symbol '-' means that there was no or only one species showing that character, preventing any computation of MPD value. When there was a '-' for all the cases tested for one trait (e.g. Colour C in the opaque zone), we withdrew the trait from the table. 
Table S6. Correlation between structural traits taken as binary traits and relation with structural features.

\begin{tabular}{|c|c|c|c|c|c|}
\hline Zone & Wing & Variable & Variable & $\mathrm{Nb}$ & $\begin{array}{c}\text { LRT } \\
\text { (type = h) }\end{array}$ \\
\hline \multirow{4}{*}{ Transparent } & \multirow{4}{*}{ Forewing and Hindwing } & \multicolumn{2}{|c|}{ presence } & 115 & $66.4 * * *$ \\
\hline & & \multicolumn{2}{|c|}{ type $\neq$} & 83 & $83.07 * * *$ \\
\hline & & \multicolumn{2}{|c|}{ insertion } & 83 & $73.3 * * *$ \\
\hline & & \multicolumn{2}{|c|}{ colouration } & 83 & $79.63 * * *$ \\
\hline \multirow{2}{*}{ Opaque } & \multirow{2}{*}{ Forewing and Hindwing } & \multicolumn{2}{|c|}{ type ${ }^{\ddagger}$} & 123 & $36.41 * * *$ \\
\hline & & \multicolumn{2}{|c|}{ insertion } & 123 & $11.61 *$ \\
\hline \multirow{2}{*}{ Opaque and Transparent } & \multirow{2}{*}{ Forewing } & \multicolumn{2}{|c|}{ type $^{\ddagger}$} & 119 & $26.26 * * *$ \\
\hline & & \multicolumn{2}{|c|}{ insertion } & 93 & 1.78 \\
\hline \multirow{2}{*}{ Opaque and Transparent } & \multirow{2}{*}{ Hindwing } & \multirow{2}{*}{\multicolumn{2}{|c|}{$\begin{array}{c}\text { type }^{\ddagger} \\
\text { insertion }\end{array}$}} & 119 & $23.85^{* * *}$ \\
\hline & & & & 90 & 2.20 \\
\hline \multirow{3}{*}{ Transparent } & \multirow{3}{*}{ Forewing } & type ${ }^{\ddagger}$ & insertion & 93 & 3.27 \\
\hline & & type $e^{\ddagger}$ & colouration & 93 & $18.79 * * *$ \\
\hline & & insertion & colouration & 93 & 6.19 \\
\hline \multirow{3}{*}{ Transparent } & \multirow{3}{*}{ Hindwing } & type $^{\ddagger}$ & insertion & 90 & 3.12 \\
\hline & & type $e^{\ddagger}$ & colouration & 90 & $17.56 * *$ \\
\hline & & insertion & colouration & 90 & $9.71 *$ \\
\hline \multirow{2}{*}{ Opaque } & Forewing & type ${ }^{\ddagger}$ & insertion & 123 & 0.13 \\
\hline & Hindwing & type ${ }^{\ddagger}$ & insertion & 123 & 0.10 \\
\hline
\end{tabular}

For each analysis, we indicate the number of species included in the analysis ( $\mathrm{Nb}$ ), the likelihood ratio value with the associated $p$-value (bold values are significant $p$-values $* p<0.05,{ }^{*} p<0.01,{ }^{* * *} p<0.001$ ) between the model with dependent traits ( 4 parameters estimated) and the model with independent traits ( 8 parameters estimated). A significant $p$-value indicated that the dependent model did significantly better than the independent model, indicating a correlated evolution between the traits (underlined in bold). To test the correlations involving type, insertion or colouration of scales in the transparent zone, we excluded the species for which scales were absent in the transparent zone. Insertion was then a binary trait (flat versus erect), as well as colouration (transparent versus coloured). ₹ For scale type, we considered that the combination piliform scales and lamellar scales was assimilated to lamellar scales and we built the binary variable (piliform scales versus (lamellar scales alone or the combination piliform scales and lamellar scales). 
Figure S4. Phylogeny-controlled correlations between structural binary traits as tested with Pagel's method. Scale type was separated into two binary variables: $p=$ presence (yes/no) and $t=t y p e$ (piliform scales versus lamellar scales alone or combination of piliform scales and lamellar scales). $\mathrm{i}$ = insertion (flat versus erect), and $c=$ colouration (transparent versus coloured). Presence of a link indicates significant correlation, between wings, between the opaque zone (grey) and the transparent zone (white) of the same wing, within a zone. In the opaque zone, scales were always present and coloured, which made impossible to test correlations involving $\mathrm{p}$ or $\mathrm{c}$ variables.

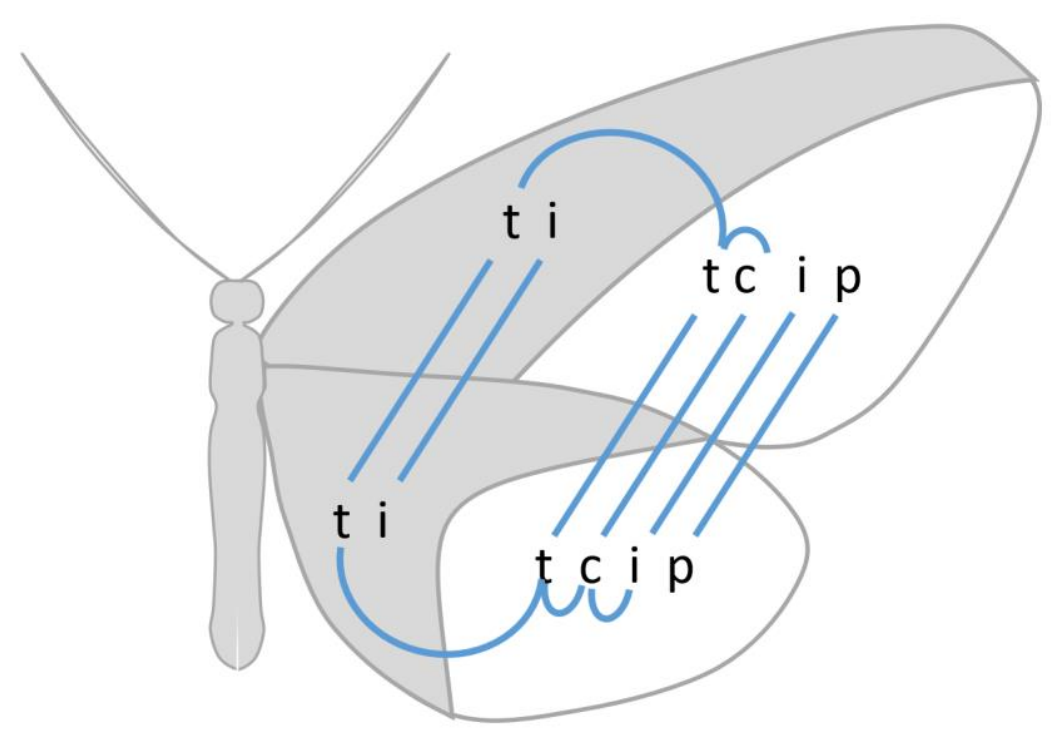


Table S7. Relationship between relative investment in transparency and wing microstructure.

\begin{tabular}{|c|c|c|c|c|}
\hline & \multirow{2}{*}{\multicolumn{2}{|c|}{ Mixed model }} & \multirow{3}{*}{$\begin{array}{c}\text { Bayesian } \\
\text { Estimate }[95 \% \mathrm{Cl}]\end{array}$} \\
\hline & & & & \\
\hline Variable & Factors & Estimate \pm se & t-value & \\
\hline \multirow{7}{*}{$\begin{array}{l}\text { Scale density in } \\
\text { the transparent } \\
\text { zone - Scale } \\
\text { density in the } \\
\text { opaque zone }\end{array}$} & Intercept & $-78.6 \pm 43.9$ & -1.79 & $-94.2[-290.7,108]$ \\
\hline & OZ Scale coverage & $-71.4 \pm 19.7$ & $-3.62 * * *$ & $-80.8[-118.3,-45]$ \\
\hline & Scale $(P>N)$ & $161.3 \pm 47.8$ & $3.37^{* * *}$ & $146.8[53.4,239.1]$ \\
\hline & Scale $((L, P L)>P)$ & $69.3 \pm 46.8$ & 1.48 & $83.6[-9,171.3]+$ \\
\hline & Colouration $(T>(C, U))$ & $149.6 \pm 45.4$ & $3.29 * * *$ & $78.1[-9.8,168.9]+$ \\
\hline & Phylogenetic variance & - & - & $45028.9[27177,67908.9]$ \\
\hline & Residual variance & - & - & $21097.9[16481.5,27063.1]$ \\
\hline \multirow{6}{*}{$\begin{array}{l}\text { Scale length in } \\
\text { the transparent } \\
\text { zone - Scale } \\
\text { length in the } \\
\text { opaque zone }\end{array}$} & Intercept & $-8.4 \pm 8$ & -1.06 & $-10.5[-39.5,16.7]$ \\
\hline & OZ Scale surface & $-0.004 \pm 0.0009$ & $-4.86 * * *$ & $-0.004[-0.006,-0.002]$ \\
\hline & Insertion $(F>E)$ & $16.5 \pm 9.4$ & 1.76 & $20.3[1.8,39.6]$ \\
\hline & Insertion $(\mathrm{U}>\mathrm{E})$ & $-102.2 \pm 11.1$ & $-9.18^{* * *}$ & $-93.6[-117.9,-68.7]$ \\
\hline & Phylogenetic variance & - & - & $584.2[0,1745.7]$ \\
\hline & Residual variance & - & - & $2975.4[2312.6,3716.1]$ \\
\hline \multirow{7}{*}{$\begin{array}{l}\text { Scale width in } \\
\text { the transparent } \\
\text { zone - Scale } \\
\text { width in the } \\
\text { opaque zone }\end{array}$} & Intercept & $-17.9 \pm 3.4$ & $-5.27 * * *$ & $-10[-28,7]$ \\
\hline & OZ Scale surface & $-0.002 \pm 0.0003$ & $-8.24 * * *$ & $-0.003[-0.003,-0.002]$ \\
\hline & Colouration $(T>(C, U))$ & $26.3 \pm 3.8$ & $6.83 * * *$ & $23.6[15.8,31.5]$ \\
\hline & Scale $(\mathrm{N}<\mathrm{P})$ & $10.4 \pm 4.1$ & $2.51^{*}$ & $13.8[5.5,22]$ \\
\hline & Scale $(P<(L, P L))$ & $19.4 \pm 4$ & $4.82 * * *$ & $19[11.2,27.1]$ \\
\hline & Phylogenetic variance & - & - & $330[157.7,554.2]$ \\
\hline & Residual variance & - & - & $184.3[137.1,243.3]$ \\
\hline \multirow{8}{*}{$\begin{array}{l}\text { Scale surface in } \\
\text { the transparent } \\
\text { zone - Scale } \\
\text { surface in the } \\
\text { opaque zone }\end{array}$} & Intercept & $-273.9 \pm 513.1$ & -0.53 & $411.4[-1890.6,2731.8]$ \\
\hline & OZ Scale surface & $-0.6 \pm 0$ & $-15.2^{* * *}$ & $-0.6[-0.7,-0.6]$ \\
\hline & Colouration $(T>(C, U))$ & $1991.6 \pm 490.3$ & $4.06 * * *$ & $2604.6[1650.1,3569.2]$ \\
\hline & Scale $(N<P)$ & $1589.7 \pm 526.9$ & $3.02 * *$ & $1622[560.8,2670.8]$ \\
\hline & Scale $(P<(L, P L))$ & $2640.1 \pm 510.5$ & $5.17^{* * *}$ & $2109.1[1099.6,3160.7]$ \\
\hline & Wing Length & $-27.5 \pm 13.1$ & $-2.09 *$ & $-24.3[-52.5,3.4] \dagger$ \\
\hline & Phylogenetic variance & - & - & 5723006 [3031930, 9058424] \\
\hline & Residual variance & - & - & 2592997 [1941769, 3448755] \\
\hline \multirow{7}{*}{$\begin{array}{l}\text { Scale coverage } \\
\text { in the } \\
\text { transparent } \\
\text { zone - Scale } \\
\text { coverage in the } \\
\text { opaque zone }\end{array}$} & Intercept & $-0.04 \pm 0.14$ & -0.29 & $0.04[-0.46,0.55]$ \\
\hline & OZ Scale coverage & $-0.85 \pm 0.07$ & $-11.92 * * *$ & $-0.82[-0.97,-0.68]$ \\
\hline & Colouration $(T>(C, U))$ & $1.2 \pm 0.14$ & $8.74 * * *$ & $1.01[0.7,1.31]$ \\
\hline & Scale $(\mathrm{N}<\mathrm{P})$ & $0.15 \pm 0.15$ & 1.01 & $0.13[-0.19,0.44]$ \\
\hline & Scale $(P<(L, P L))$ & $0.36 \pm 0.14$ & $2.62^{*}$ & $0.36[0.06,0.65]$ \\
\hline & Phylogenetic variance & - & - & $0.25[0.09,0.44]$ \\
\hline & Residual variance & - & - & $0.37[0.29,0.47]$ \\
\hline
\end{tabular}

Retained classic mixed model and Bayesian phylogenetically controlled mixed model. All factors relating to scale concerned scale from the transparent zone, except when stated otherwise, with $\mathrm{OZ}=$ opaque zone. $\mathrm{FW}$ = forewing, $\mathrm{HW}=$ Hindwing, scale type $(\mathrm{P}=$ piliform scales $\mathrm{L}=$ lamellar scales, $\mathrm{PL}=$ piliform scales and lamellar scales in combination, $\mathrm{N}=$ none), insertion ( $\mathrm{F}=$ flat, $\mathrm{E}=$ erect, $\mathrm{U}=$ unknown), and scale colouration $(T=$ transparent, $\mathrm{C}=$ coloured, $\mathrm{U}=$ unknown). Scale surface is in $\mu \mathrm{m}^{2}$ while scale coverage is computed with scale surface expressed in $\mathrm{mm}^{2}$, in order to get readable results. Bold values are statistically important factors associated with $95 \% \mathrm{Cl}$ excluding zero in Bayesian models, less important factors are associated with $90 \% \mathrm{Cl}$ excluding zero (with symbol $t$ ) and $p$-values ( ${ }^{*} p<0.05 ;{ }^{* *} p<0.01 ; * * * * p<0.001$ ) for the mixed model adjusted for type III sums of squares. Results concerning PS strategies are based on the analyses of the dimensions of the lamellar scales, not the piliform scales of the PS combination. 
Figure S5. Relative investment in transparency measuring to what extent scale structural features in the transparent zone are modified relatively to the opaque zone. Plots detail the relationships between wing clearwing area and difference in scale density (a), between structural strategy and difference in scale density (b), length (c), width (d), surface (e) and coverage (surface * density) (f). A structural strategy is defined as the combination of scale type, insertion and colouration. Type is $P=$ piliform scales, $L=$ lamellar scales (piliform scales and lamellar scales were assimilated to lamellar scales), insertion is $E=$ erect, $F=$ flat and colouration is $\mathrm{C}=$ coloured, $\mathrm{T}=$ transparent. The $\mathrm{N}$ strategy has no scales, no insertion and no colouration. $\mathrm{A}$ few outliers were withdrawn from plots for clarity reasons but not from analyses. Notice that in the PL strategies, measured structural features concern the lamellar scales and not the piliform scales of these PL strategies.
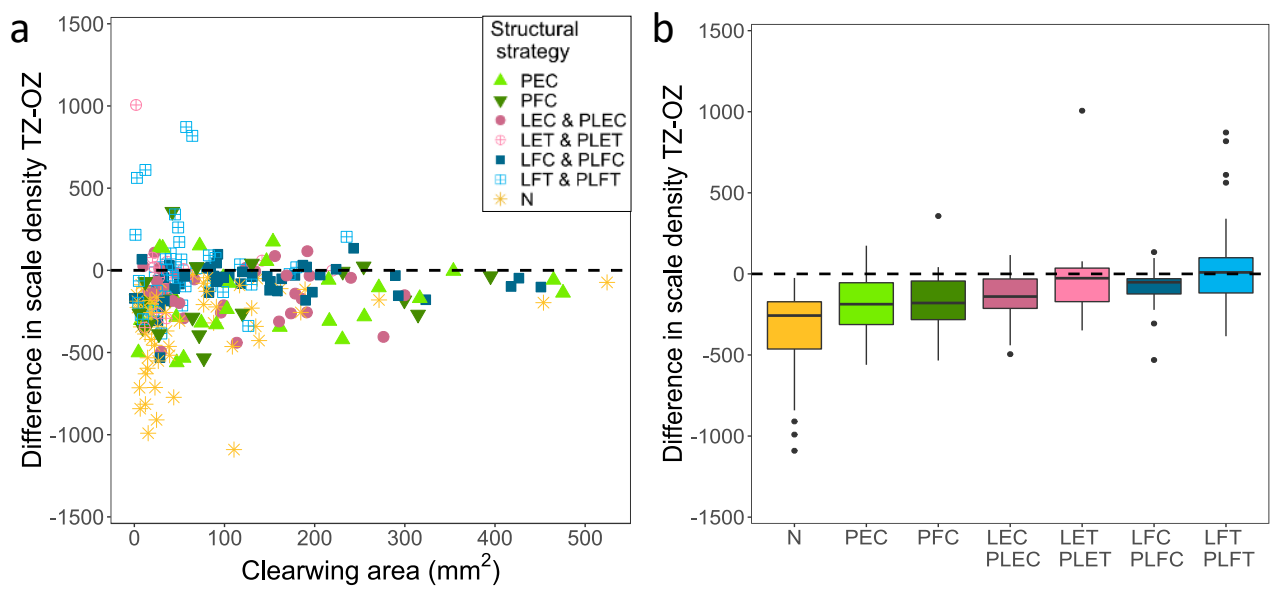

C
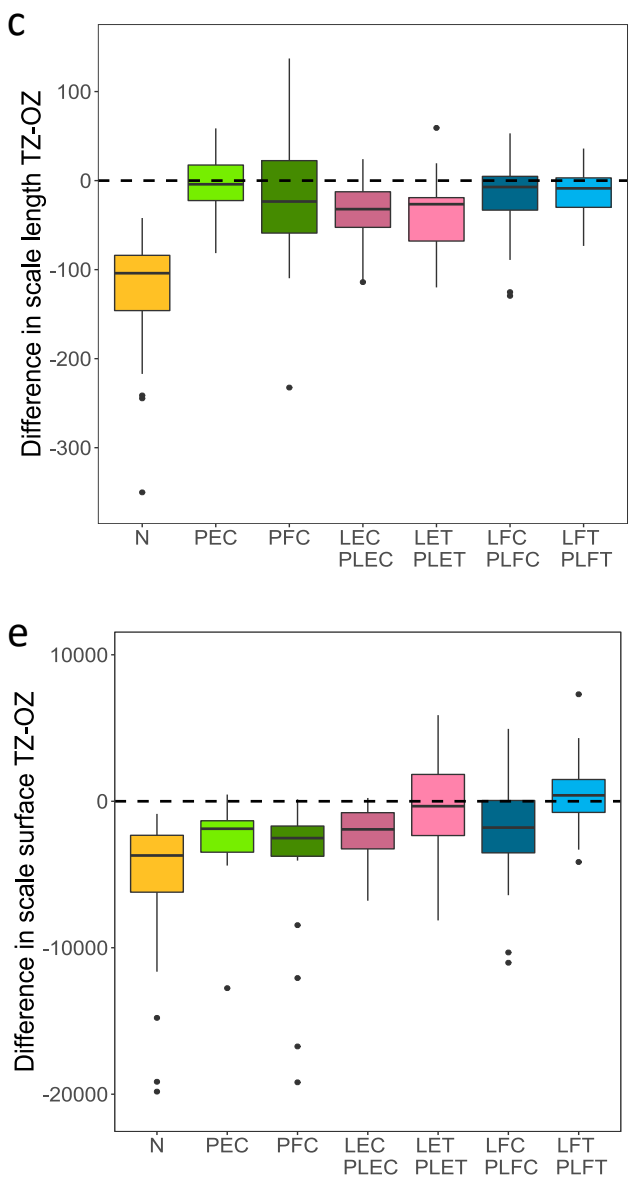

d
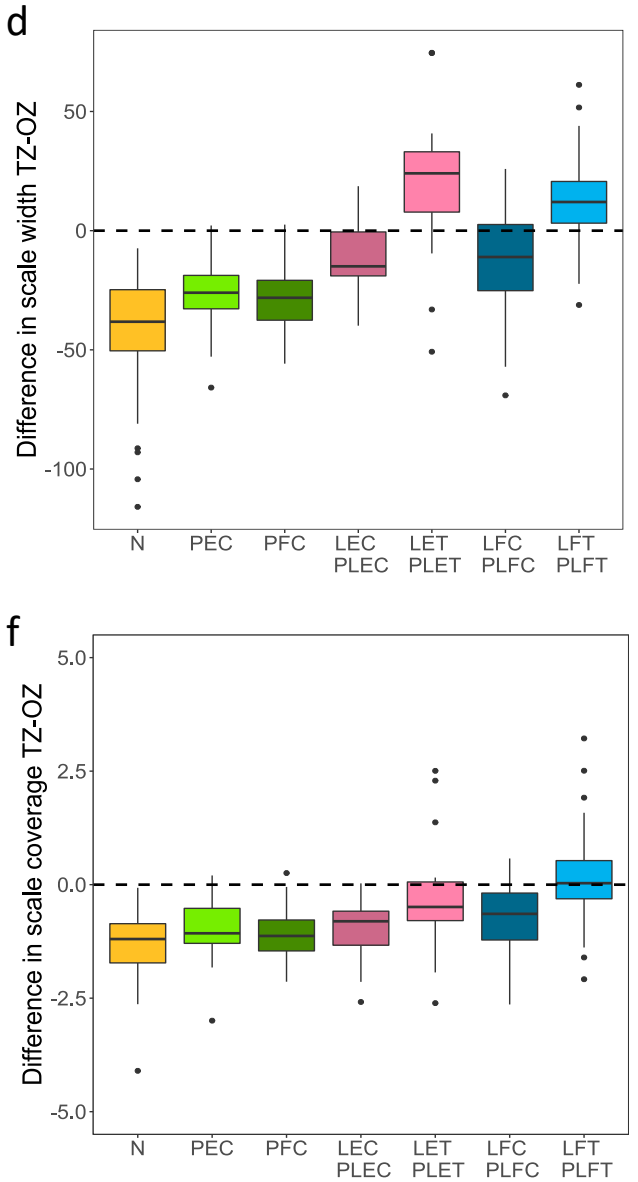
Table S8. Relationships between optical parameters.

\begin{tabular}{ccccc} 
& & \multicolumn{2}{c}{ Mixed model } & Bayesian \\
\cline { 3 - 5 } Variable & Factor & Estimate $\pm \mathrm{se}$ & t-value & Estimate $[95 \% \mathrm{Cl}]$ \\
\cline { 3 - 5 } & intercept & $8.5 \pm 2.7$ & $3.12^{* *}$ & $0.5[-27.6,28.8]$ \\
Mean & Proportion of UV transmittance\# & $\mathbf{2 . 7 3 \pm 0 . 1 3}$ & $\mathbf{2 0 . 9 8 ^ { * * * }}$ & $\mathbf{3 . 1 5}[\mathbf{2 . 9 6 , 3 . 3 6 ]}$ \\
transmittance & (FW $>\mathrm{HW})$ & $\mathbf{1 . 9 \pm 0 . 7}$ & $\mathbf{2 . 8 7 ^ { * * }}$ & $\mathbf{1 . 9}[\mathbf{1 . 2 4}, \mathbf{2 . 6}]$ \\
& Phylogenetic variance & & & $1096[833.3,1430.4]$ \\
& Residual variance & & & $42.6[39.3,46.4]$ \\
\hline
\end{tabular}

Retained classic mixed model and Bayesian phylogenetically controlled mixed model. We took all 10 measurements per species. We took species and wing within species as random factors in classic mixed models and species as random factor in Bayesian models. $\mathrm{FW}=$ forewing, $\mathrm{HW}=$ Hindwing. Bold values are statistically important factors associated with $95 \% \mathrm{Cl}$ excluding zero in Bayesian models, less important factors are associated with $90 \% \mathrm{Cl}$ excluding zero (with symbol $t$ ) and $p$-values ( ${ }^{*} p<0.05 ;{ }^{* *} p<0.01$; $\mathrm{p}<0.001$ ) for the mixed model adjusted for type III sums of squares. \# Proportion of UV transmittance was multiplied by 100 before analysis. 
Table S9. Relationship between mean transmittance and visual contrasts, for a UVS avian predator in three illuminants and visual conditions.

\begin{tabular}{|c|c|c|c|c|c|c|c|c|c|c|}
\hline \multirow{3}{*}{$\begin{array}{c}\text { Mean } \\
\text { transmittance }\end{array}$} & \multirow[b]{3}{*}{ Factor } & \multicolumn{3}{|c|}{ sky background } & \multicolumn{3}{|c|}{ leaf background } & \multicolumn{3}{|c|}{ trunk background } \\
\hline & & \multicolumn{2}{|c|}{ mixed model } & \multirow{2}{*}{$\begin{array}{c}\text { Bayesian } \\
\text { Estimate }[95 \% \mathrm{Cl}] \\
\end{array}$} & \multicolumn{2}{|c|}{ mixed model } & \multirow{2}{*}{$\begin{array}{c}\text { Bayesian } \\
\text { Estimate }[95 \% \mathrm{Cl}] \\
\end{array}$} & \multicolumn{2}{|c|}{ mixed model } & \multirow{2}{*}{$\begin{array}{c}\text { Bayesian } \\
\text { Estimate }[95 \% \mathrm{Cl}] \\
\end{array}$} \\
\hline & & Estimate \pm se & t-value & & Estimate \pm se & t-value & & Estimate \pm se & t-value & \\
\hline \multirow{9}{*}{$\begin{array}{l}\text { woodland } \\
\text { shade }\end{array}$} & intercept & $85.7 \pm 0.7$ & $117^{* * *}$ & $84.2[76.8,91.6]$ & $88.4 \pm 0.8$ & $117^{* * *}$ & $86.6[79.4,93.7]$ & $88 \pm 0.7$ & $118^{* * *}$ & $86.2[79.1,93.3]$ \\
\hline & CC & $-9.6 \pm 0.5$ & $-21 * * *$ & $-10.2[-11,-9.3]$ & $-29.4 \pm 1.3$ & $-23 * * *$ & $-30.4[-32.7,-28.1]$ & $-21.4 \pm 0.9$ & $-23 * * *$ & $-22.2[-23.9,-20.5]$ \\
\hline & $\mathrm{BC}$ & $-11.8 \pm 0.2$ & $-61 * * *$ & $-10.9[-11.3,-10.6]$ & $-16.2 \pm 0.3$ & $-61 * * *$ & $-14.9[-15.4,-14.5]$ & $-14.2 \pm 0.2$ & $-61 * * *$ & $-13.1[-13.5,-12.7]$ \\
\hline & $(\mathrm{FW}>\mathrm{HW})$ & $2.2 \pm 0.5$ & $4 * * *$ & $1.2[0.6,1.8]$ & $2.6 \pm 0.6$ & $5^{* * *}$ & $1.4[0.8,2.1]$ & $2.6 \pm 0.6$ & $5 * * *$ & $1.4[0.8,2.1]$ \\
\hline & $\mathrm{CCXBC}$ & $1.8 \pm 0.1$ & $20 * * *$ & $1.7[1.6,1.9]$ & $7.3 \pm 0.3$ & $22^{* * *}$ & $6.9[6.3,7.4]$ & $4.8 \pm 0.2$ & $22 * * *$ & $4.4[4.1,4.8]$ \\
\hline & $\mathrm{CCx}(\mathrm{FW}>\mathrm{HW})$ & $0.8 \pm 0.3$ & $3^{* *}$ & $0.5[0.2,0.8]$ & $2 \pm 0.8$ & $2^{*}$ & $0.9[0.1,1.8]$ & $1.5 \pm 0.6$ & $3^{*}$ & $0.7[0.1,1.3]$ \\
\hline & $\mathrm{BCx}((\mathrm{FW}>\mathrm{HW})$ & $-1.5 \pm 0.2$ & $-9 * * *$ & $-0.8[-1,-0.6]$ & $-2 \pm 0.2$ & $-9 * * *$ & $-1.1[-1.4,-0.8]$ & $-1.8 \pm 0.2$ & $-9 * * *$ & $-1[-1.2,-0.7]$ \\
\hline & phyl var & - & - & $78.4[58.6,103.4]$ & - & - & $72.7[54.2,95.7]$ & - & - & $71.7[53.5,94.4]$ \\
\hline & res var & - & - & $8.1[7.4,8.8]$ & - & - & $8.2[7.5,8.9]$ & - & - & $8.2[7.5,9]$ \\
\hline \multirow{9}{*}{ forest shade } & intercept & $86.7 \pm 0.7$ & $123^{* * *}$ & $85.2[78.2,92]$ & $89 \pm 0.7$ & $121 * * *$ & $87.1[80.2,94]$ & $88.6 \pm 0.7$ & $123^{* * *}$ & $86.6[79.8,93.5]$ \\
\hline & CC & $-18.4 \pm 0.8$ & $-24 * * *$ & $-19.2[-20.6,-17.8]$ & $-64.2 \pm 2.5$ & $-25 * * *$ & $-66[-70.6,-61.3]$ & $-44.5 \pm 1.8$ & $-25 * * *$ & $-45.7[-48.9,-42.4]$ \\
\hline & $\mathrm{BC}$ & $-14.9 \pm 0.2$ & $-64 * * *$ & $-13.9[-14.3,-13.4]$ & $-21.7 \pm 0.3$ & $-63 * * *$ & $-20[-20.6,-19.3]$ & $-19.5 \pm 0.3$ & $-63 * * *$ & $-17.9[-18.5,-17.4]$ \\
\hline & $(\mathrm{FW}>\mathrm{HW})$ & $2.2 \pm 0.5$ & $4^{* * *}$ & $1.2[0.6,1.8]$ & $2.5 \pm 0.6$ & $4 * * *$ & $1.4[0.7,2]$ & $2.5 \pm 0.6$ & $4 * * *$ & $1.4[0.8,2]$ \\
\hline & CCXBC & $4.2 \pm 0.2$ & $21 * * *$ & $4.1[3.7,4.4]$ & $21.4 \pm 0.9$ & $24 * * *$ & $19.7[18.2,21.2]$ & $13.4 \pm 0.6$ & $24 * * *$ & $12.4[11.4,13.3]$ \\
\hline & $\mathrm{CCx}(\mathrm{FW}>\mathrm{HW})$ & $1.3 \pm 0.5$ & $3^{* *}$ & $0.7[0.2,1.2]$ & $3.8 \pm 1.7$ & $2^{*}$ & $1.7[-0.1,3.5]$ & $2.6 \pm 1.2$ & $2^{*}$ & $1.1[-0.1,2.4]$ \\
\hline & $\mathrm{BCx}(\mathrm{FW}>\mathrm{HW})$ & $-1.7 \pm 0.2$ & $-9 * * *$ & $-1[-1.2,-0.7]$ & $-2.6 \pm 0.3$ & $-9 * * *$ & $-1.4[-1.7,-1]$ & $-2.3 \pm 0.3$ & $-9 * * *$ & $-1.2[-1.6,-0.9]$ \\
\hline & phyl var & - & - & $68.7[51.3,90.7]$ & - & - & $67.5[50.3,89.1]$ & - & - & $66.6[49.7,88]$ \\
\hline & res var & - & - & $7.5[6.9,8.2]$ & - & - & $7.8[7.2,8.5]$ & - & - & $7.9[7.2,8.6]$ \\
\hline \multirow{9}{*}{ large gap } & intercept & $84.8 \pm 0.7$ & $115^{* * *}$ & $83.2[75.4,90.9]$ & $86.9 \pm 0.7$ & $119 * * *$ & $85.2[78.1,92.3]$ & $86.5 \pm 0.7$ & $119 * * *$ & $84.7[77.5,91.8]$ \\
\hline & CC & $-6 \pm 0.2$ & $-24 * * *$ & $-6.2[-6.6,-5.7]$ & $-13 \pm 0.5$ & $-25 * * *$ & $-13.3[-14.3,-12.4]$ & $-9.1 \pm 0.4$ & $-25 * * *$ & $-9.4[-10,-8.7]$ \\
\hline & $\mathrm{BC}$ & $-8.7 \pm 0.1$ & $-60 * * *$ & $-8.1[-8.4,-7.8]$ & $-6.2 \pm 0.1$ & $-62 * * *$ & $-5.7[-5.8,-5.5]$ & $-5.8 \pm 0.1$ & $-62 * * *$ & $-5.3[-5.5,-5.1]$ \\
\hline & $(\mathrm{FW}>\mathrm{HW})$ & $2.1 \pm 0.5$ & $4 * * *$ & $1.1[0.5,1.7]$ & $2.2 \pm 0.6$ & $4 * * *$ & $1.2[0.6,1.9]$ & $2.2 \pm 0.6$ & $4 * * *$ & $1.3[0.6,1.9]$ \\
\hline & CCXBC & $0.8 \pm 0$ & $22 * * *$ & $0.8[0.7,0.9]$ & $1.3 \pm 0.1$ & $24 * * *$ & $1.2[1.1,1.2]$ & $0.8 \pm 0$ & $24 * * *$ & $0.8[0.7,0.8]$ \\
\hline & $\mathrm{CCx}(\mathrm{FW}>\mathrm{HW})$ & $0.4 \pm 0.2$ & $3^{* *}$ & $0.3[0.1,0.4]$ & $0.7 \pm 0.3$ & $2^{*}$ & $0.3[-0.1,0.7]$ & $0.5 \pm 0.2$ & $2^{*}$ & $0.2[-0.03,0.5]$ \\
\hline & $\mathrm{BCx}(\mathrm{FW}>\mathrm{HW})$ & $-1.1 \pm 0.1$ & $-8 * * *$ & $-0.6[-0.8,-0.4]$ & $-0.7 \pm 0.1$ & $-8 * * *$ & $-0.4[-0.5,-0.3]$ & $-0.7 \pm 0.1$ & $-8^{* * *}$ & $-0.4[-0.5,-0.3]$ \\
\hline & phyl var & - & - & $86.1[64.3,113.5]$ & - & - & $72.7[54.4,95.8]$ & - & - & $73.3[54.9,96.6]$ \\
\hline & res var & - & - & $8.4[7.7,9.2]$ & - & - & $8.1[7.4,8.8]$ & - & - & $8.2[7.5,8.9]$ \\
\hline
\end{tabular}




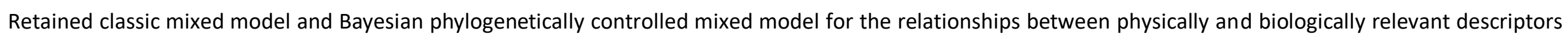

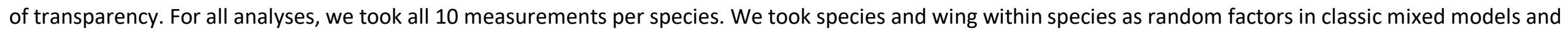

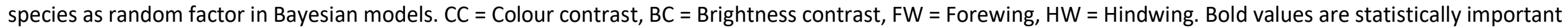

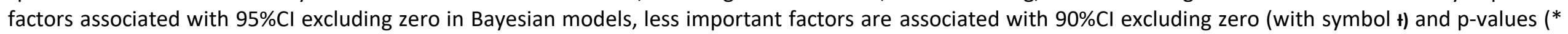

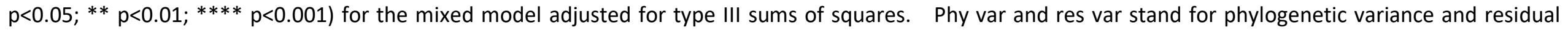
variance respectively. 
Table S10. Relationship between mean transmittance and visual contrasts, for a VS avian predator in various visual conditions.

\begin{tabular}{|c|c|c|c|c|c|c|c|c|c|c|}
\hline \multirow{3}{*}{$\begin{array}{c}\text { Mean } \\
\text { transmittance }\end{array}$} & \multirow[b]{3}{*}{ Factor } & \multicolumn{3}{|c|}{ sky background } & \multicolumn{3}{|c|}{ leaf background } & \multicolumn{3}{|c|}{ trunk background } \\
\hline & & \multicolumn{2}{|c|}{ mixed model } & \multirow{2}{*}{$\frac{\text { Bayesian }}{\text { Estimate }[95 \% \mathrm{Cl}]}$} & \multicolumn{2}{|c|}{ mixed model } & \multirow{2}{*}{$\frac{\text { Bayesian }}{\text { Estimate }[95 \% \mathrm{Cl}]}$} & \multicolumn{2}{|c|}{ mixed model } & \multirow{2}{*}{$\frac{\text { Bayesian }}{\text { Estimate }[95 \% \mathrm{Cl}]}$} \\
\hline & & Estimate \pm se & t-value & & Estimate \pm se & t-value & & Estimate \pm se & t-value & \\
\hline \multirow{9}{*}{$\begin{array}{l}\text { woodland } \\
\text { shade }\end{array}$} & intercept & $86.1 \pm 0.7$ & $121 * * *$ & $84.5[77.4,91.4]$ & $88.4 \pm 0.7$ & $122^{* * *}$ & $86.5[79.6,93.3]$ & $88.1 \pm 0.7$ & $122 * * *$ & $86.2[79.3,93]$ \\
\hline & $\mathrm{CC}$ & $-12.4 \pm 0.5$ & $-23 * * *$ & $-13[-13.9,-12]$ & $-38 \pm 1.6$ & $-24 * * *$ & $-39[-41.7,-36.1]$ & $-26 \pm 1.1$ & $-24 * * *$ & $-26.7[-28.7,-24.7]$ \\
\hline & BC & $-12.1 \pm 0.2$ & $-62 * * *$ & $-11.2[-11.5,-10.8]$ & $-16.5 \pm 0.3$ & $-63 * * *$ & $-15.1[-15.6,-14.7]$ & $-14.5 \pm 0.2$ & $-62 * * *$ & $-13.3[-13.7,-12.9]$ \\
\hline & $(\mathrm{FW}>\mathrm{HW})$ & $2.3 \pm 0.5$ & $4 * * *$ & $1.3[0.7,1.9]$ & $2.6 \pm 0.6$ & $5 * * *$ & $1.5[0.8,2.1]$ & $2.6 \pm 0.6$ & $5 * * *$ & $1.5[0.9,2.1]$ \\
\hline & $\mathrm{CCxBC}$ & $2.4 \pm 0.1$ & $21 * * *$ & $2.3[2.1,2.5]$ & $9.8 \pm 0.4$ & $24 * * *$ & $8.9[8.2,9.6]$ & $5.9 \pm 0.3$ & $23^{* * *}$ & $5.4[5,5.9]$ \\
\hline & $\operatorname{cCx}((\mathrm{FW}>\mathrm{HW})$ & $0.9 \pm 0.3$ & $3^{*}$ & $0.4[0,0.8]$ & $2.1 \pm 1$ & $2^{*}$ & $0.6[0.5,1.7]$ & $1.5 \pm 0.7$ & $2^{*}$ & $0.5[0.3,1.3]$ \\
\hline & $\mathrm{BCx}(\mathrm{FW}>\mathrm{HW})$ & $-1.4 \pm 0.2$ & $-9 * * *$ & $-0.8[-1,-0.6]$ & $-2 \pm 0.2$ & $-9 * * *$ & $-1.1[-1.3,-0.8]$ & $-1.8 \pm 0.2$ & $-9 * * *$ & $-0.9[-1.2,-0.7]$ \\
\hline & phyl var & - & - & $70.5[52.8,92.7]$ & - & - & $66.1[49.4,87.3]$ & - & - & $66.7[49.8,88]$ \\
\hline & res var & - & - & $7.8[7.2,8.5]$ & - & - & $8[7.3,8.7]$ & - & - & $8.1[7.4,8.8]$ \\
\hline \multirow{9}{*}{ forest shade } & intercept & $86.9 \pm 0.7$ & $127^{* * *}$ & $85.2[78.5,91.8]$ & $89 \pm 0.7$ & $128^{* * *}$ & $86.8[79.9,93.7]$ & $88.7 \pm 0.7$ & $127^{* * *}$ & $86.5[79.6,93.5]$ \\
\hline & CC & $-23.3 \pm 0.9$ & $-25 * * *$ & $-24.1[-25.8,-22.4]$ & $-82.1 \pm 2.9$ & $-29 * * *$ & $-82.2[-87.6,-76.7]$ & $-54.4 \pm 1.9$ & $-28 * * *$ & $-54.5[-58.1,-50.8]$ \\
\hline & BC & $-15.1 \pm 0.2$ & $-65 * * *$ & $-14[-14.4,-13.6]$ & $-21.8 \pm 0.3$ & $-66 * * *$ & $-20[-20.6,-19.4]$ & $-19.5 \pm 0.3$ & $-65 * * *$ & $-18[-18.5,-17.4]$ \\
\hline & $(\mathrm{FW}>\mathrm{HW})$ & $2.2 \pm 0.5$ & $4 * * *$ & $1.3[0.7,1.8]$ & $2.9 \pm 0.5$ & $6 * * *$ & $1.5[1,2.1]$ & $3 \pm 0.5$ & $6 * * *$ & $1.6[1,2.1]$ \\
\hline & $\mathrm{CCxBC}$ & $5.5 \pm 0.2$ & $23 * * *$ & $5.2[4.8,5.6]$ & $27.1 \pm 1.1$ & $26 * * *$ & $24.6[22.7,26.4]$ & $16.2 \pm 0.7$ & $25^{* * *}$ & $14.7[13.6,15.8]$ \\
\hline & $\operatorname{CCx}((\mathrm{FW}>\mathrm{HW})$ & $1.4 \pm 0.6$ & $2^{*}$ & $0.6[0.1,1.2]$ & - & - & - & - & - & - \\
\hline & $\mathrm{BCx}(\mathrm{FW}>\mathrm{HW})$ & $-1.7 \pm 0.2$ & $-8 * * *$ & $-0.9[-1.2,-0.7]$ & $-2.3 \pm 0.3$ & $-8^{* * *}$ & $-1.3[-1.6,-0.9]$ & $-2.1 \pm 0.3$ & $-8^{* * *}$ & $-1.2[-1.5,-0.8]$ \\
\hline & phyl var & - & - & $63.1[47.2,83.2]$ & - & - & $63[47,83.6]$ & - & - & $64[47.7,85]$ \\
\hline & res var & - & - & $7.3[6.7,7.9]$ & - & - & $7.7[7.1,8.4]$ & - & - & $7.8[7.2,8.5]$ \\
\hline \multirow{9}{*}{ large gap } & intercept & $85 \pm 0.7$ & $119 * * *$ & $83.2[75.7,90.5]$ & $86.9 \pm 0.7$ & $124^{* * *}$ & $84.9[77.7,92.1]$ & $86.5 \pm 0.7$ & $123^{* * *}$ & $84.5[77.3,91.8]$ \\
\hline & CC & $-7.2 \pm 0.3$ & $-25 * * *$ & $-7.4[-7.9,-6.9]$ & $-15.8 \pm 0.6$ & $-28 * * *$ & $-15.8[-16.9,-14.7]$ & $-10.7 \pm 0.4$ & $-28 * * *$ & $-10.8[-11.5,-10]$ \\
\hline & BC & $-8.9 \pm 0.1$ & $-61 * * *$ & $-8.2[-8.5,-7.9]$ & $-6.2 \pm 0.1$ & $-64 * * *$ & $-5.7[-5.8,-5.5]$ & $-5.8 \pm 0.1$ & $-63 * * *$ & $-5.3[-5.5,-5.1]$ \\
\hline & $(\mathrm{FW}>\mathrm{HW})$ & $2.1 \pm 0.5$ & $4 * * *$ & $1.2[0.6,1.8]$ & $2.6 \pm 0.5$ & $5 * * *$ & $1.4[0.8,2]$ & $2.7 \pm 0.5$ & $5^{* * *}$ & $1.4[0.9,2]$ \\
\hline & $\mathrm{CCxBC}$ & $1 \pm 0$ & $24 * * *$ & $1[0.9,1]$ & $1.5 \pm 0.1$ & $26 * * *$ & $1.4[1.3,1.5]$ & $1 \pm 0$ & $25^{* * *}$ & $0.9[0.8,1]$ \\
\hline & $\mathrm{CCx}(\mathrm{FW}>\mathrm{HW})$ & $0.5 \pm 0.2$ & $2^{*}$ & $0.2[0.0 .4]$ & - & - & - & - & - & - \\
\hline & $\mathrm{BCx}(\mathrm{FW}>\mathrm{HW})$ & $-1.1 \pm 0.1$ & $-8 * * *$ & $-0.6[-0.7,-0.4]$ & $-0.6 \pm 0.1$ & $-8 * * *$ & $-0.4[-0.5,-0.2]$ & $-0.6 \pm 0.1$ & $-8 * * *$ & $-0.3[-0.4,-0.2]$ \\
\hline & phyl var & - & - & $79.2[59.2,104.2]$ & - & - & $69.5[52,92]$ & - & - & $71.3[53.3,94.4]$ \\
\hline & res var & - & - & $8.2[7.5,8.9]$ & - & - & $8[7.4,8.8]$ & - & - & $8.1[7.5,8.9]$ \\
\hline
\end{tabular}




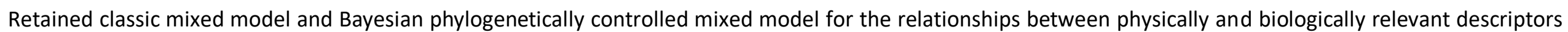

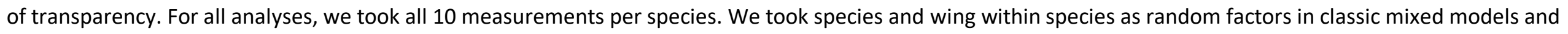

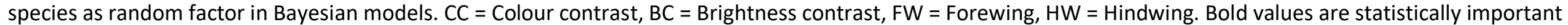

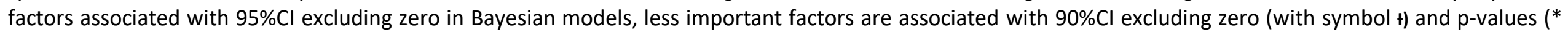

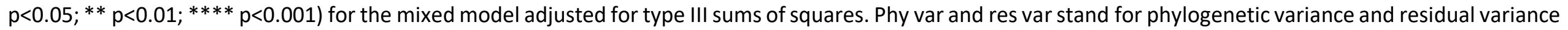
respectively. 
Table S11. Relationship between the proportion of UV transmittance $\#$ and visual contrasts, for a UVS avian predator in various visual conditions.

\begin{tabular}{|c|c|c|c|c|c|c|c|c|c|c|}
\hline & \multirow[b]{3}{*}{ Factor } & \multicolumn{3}{|c|}{ sky background } & \multicolumn{3}{|c|}{ leaf background } & \multicolumn{3}{|c|}{ trunk background } \\
\hline & & \multicolumn{2}{|c|}{ mixed model } & \multirow{2}{*}{$\begin{array}{c}\text { Bayesian } \\
\text { Estimate }[95 \% \mathrm{Cl}]\end{array}$} & \multicolumn{2}{|c|}{ mixed model } & \multirow{2}{*}{$\begin{array}{c}\text { Bayesian } \\
\text { Estimate }[95 \% \mathrm{Cl}]\end{array}$} & \multicolumn{2}{|c|}{ mixed model } & \multirow{2}{*}{$\begin{array}{c}\text { Bayesian } \\
\text { Estimate }[95 \% \mathrm{Cl}]\end{array}$} \\
\hline & & Estimate \pm se & t-value & & Estimate \pm se & t-value & & Estimate \pm se & t-value & \\
\hline \multirow{6}{*}{$\begin{array}{l}\text { woodland } \\
\text { shade }\end{array}$} & intercept & $23.9 \pm 0.2$ & $112^{* * *}$ & $23.5[20.4,26.6]$ & $23.9 \pm 0.2$ & $123 * * *$ & $23.6[20.9,26.3]$ & $23.7 \pm 0.2$ & $119 * * *$ & $23.36[20.55,26.18]$ \\
\hline & $\mathrm{CC}$ & $-5.8 \pm 0.1$ & $-64 * * *$ & $-5.64[-5.83,-5.46]$ & $-15.6 \pm 0.3$ & $-62 * * *$ & $-15.25[-15.78,-14.72]$ & $-11.4 \pm 0.2$ & $-58 * * *$ & $-11.12[-11.54,-10.7]$ \\
\hline & BC & $-0.5 \pm 0$ & $-17^{* * *}$ & $-0.61[-0.68,-0.55]$ & $-0.6 \pm 0$ & $-13^{* * *}$ & $-0.73[-0.82,-0.64]$ & $-0.6 \pm 0$ & $-13^{* * *}$ & $-0.7[-0.7,-0.6]$ \\
\hline & $\mathrm{CCxBC}$ & $0.3 \pm 0$ & $17^{* * *}$ & $0.31[0.27,0.34]$ & $1 \pm 0.1$ & $15^{* * *}$ & $1.06[0.94,1.19]$ & $0.7 \pm 0$ & $14 * * *$ & $0.7[0.61,0.78]$ \\
\hline & phyl var & - & - & $13.3[10,17.3]$ & - & - & $10.3[7.8,13.4]$ & - & - & $11[8.4,14.3]$ \\
\hline & res var & - & - & $0.32[0.29,0.35]$ & - & - & $0.36[0.33,0.39]$ & - & - & $0.4[0.37,0.43]$ \\
\hline \multirow{6}{*}{ forest shade } & intercept & $23.8 \pm 0.2$ & $115^{* * *}$ & $23.41[20.49,26.34]$ & $23.7 \pm 0.2$ & $122^{* * *}$ & $23.44[20.71,26.19]$ & $23.5 \pm 0.2$ & $117^{* * *}$ & $23.17[20.33,26.04]$ \\
\hline & CC & $-10.1 \pm 0.2$ & $-59 * * *$ & $-9.92[-10.27,-9.56]$ & $-31.9 \pm 0.6$ & $-57^{* * *}$ & $-31.3[-32.48,-30.13]$ & $-21.9 \pm 0.4$ & $-53 * * *$ & $-21.38[-22.25,-20.51]$ \\
\hline & BC & $-0.6 \pm 0$ & $-15^{* * *}$ & $-0.7[-0.8,-0.7]$ & $-0.8 \pm 0.1$ & $-12^{* * *}$ & $-0.9[-1.1,-0.8]$ & $-0.7 \pm 0.1$ & $-11^{* * *}$ & $-0.8[-1,-0.7]$ \\
\hline & $\mathrm{CCxBC}$ & $0.6 \pm 0$ & $15^{* * *}$ & $0.67[0.58,0.75]$ & $2.8 \pm 0.2$ & $14 * * *$ & $2.98[2.6,3.35]$ & $1.8 \pm 0.1$ & $13 * * *$ & $1.85[1.6,2.09]$ \\
\hline & phyl var & - & - & $11.9[9,15.5]$ & - & - & $10.5[8,13.6]$ & - & - & $11.3[8.7,14.7]$ \\
\hline & res var & - & - & $0.36[0.33,0.4]$ & - & - & $0.41[0.38,0.45]$ & - & - & $0.47[0.43,0.51]$ \\
\hline \multirow{6}{*}{ large gap } & intercept & $23.4 \pm 0.2$ & $110^{* * *}$ & $23[19.9,26.1]$ & $23.3 \pm 0.2$ & $119 * * *$ & $23.1[20.26,25.96]$ & $23.2 \pm 0.2$ & $115^{* * *}$ & $22.91[19.97,25.86]$ \\
\hline & $\mathrm{CC}$ & $-3.1 \pm 0.1$ & $-59 * * *$ & $-3.04[-3.15,-2.93]$ & $-6.3 \pm 0.1$ & $-53 * * *$ & $-6.16[-6.41,-5.92]$ & $-4.4 \pm 0.1$ & $-51 * * *$ & $-4.3[-4.49,-4.12]$ \\
\hline & $\mathrm{BC}$ & $-0.4 \pm 0$ & $-14 * * *$ & $-0.4[-0.5,-0.4]$ & $-0.2 \pm 0$ & $-11 * * *$ & $-0.3[-0.3,-0.2]$ & $-0.2 \pm 0$ & $-11^{* * *}$ & $-0.2[-0.3,-0.2]$ \\
\hline & $\mathrm{CCXBC}$ & $0.1 \pm 0$ & $17^{* * *}$ & $0.13[0.12,0.15]$ & $0.2 \pm 0$ & $14 * * *$ & $0.17[0.15,0.19]$ & $0.1 \pm 0$ & $13 * * *$ & $0.11[0.1,0.13]$ \\
\hline & phyl var & - & - & $13.24[10.05,17.22]$ & - & - & $11.3[8.7,14.7]$ & - & - & $12.1[9.2,15.6]$ \\
\hline & res var & - & - & $0.39[0.36,0.43]$ & - & - & $0.48[0.44,0.52]$ & - & - & $0.5[0.46,0.55]$ \\
\hline
\end{tabular}

Retained classic mixed model and Bayesian phylogenetically controlled mixed model for the relationships between physically and biologically relevant descriptors of transparency. For all analyses, we took all 10 measurements per species. \# Proportion of UV transmittance was multiplied by 100 before analysis. We took species and wing within species as random factors in classic mixed models and species as random factor in Bayesian models. $C C=C$ Colour contrast, BC $=\mathrm{Brightness}$ contrast. Bold values are statistically important factors associated with $95 \% \mathrm{Cl}$ excluding zero in Bayesian models, less important factors are associated with $90 \% \mathrm{Cl}$ excluding zero (with symbol $t$ ) and $p$-values $\left(* p<0.05 ;{ }^{* *} p<0.01 ; * * * * 0.001\right)$ for the mixed model adjusted for type III sums of squares. Phy var and res var stand for phylogenetic variance and residual variance respectively. 
Table S12. Relationship between proportional UV transmittance ${ }^{\#}$ and visual contrasts, for a VS avian predator in three illuminants and visual conditions.

\begin{tabular}{|c|c|c|c|c|c|c|c|c|c|c|}
\hline & \multirow[b]{3}{*}{ Factor } & \multicolumn{3}{|c|}{ sky background } & \multicolumn{3}{|c|}{ leaf background } & \multicolumn{3}{|c|}{ trunk background } \\
\hline & & \multicolumn{2}{|c|}{ mixed model } & \multirow{2}{*}{$\begin{array}{c}\text { Bayesian } \\
\text { Estimate }[95 \% \mathrm{Cl}]\end{array}$} & \multicolumn{2}{|c|}{ mixed model } & \multirow{2}{*}{$\begin{array}{c}\text { Bayesian } \\
\text { Estimate }[95 \% \mathrm{Cl}]\end{array}$} & \multicolumn{2}{|c|}{ mixed model } & \multirow{2}{*}{$\begin{array}{c}\text { Bayesian } \\
\text { Estimate }[95 \% \mathrm{Cl}]\end{array}$} \\
\hline & & Estimate \pm se & t-value & & Estimate \pm se & t-value & & Estimate \pm se & t-value & \\
\hline \multirow{6}{*}{$\begin{array}{l}\text { woodland } \\
\text { shade }\end{array}$} & intercept & $23.4 \pm 0.2$ & $119 * * *$ & $23.07[20.22,25.91]$ & $23.3 \pm 0.2$ & $115^{* * *}$ & $23.03[20.05,26.03]$ & $23.2 \pm 0.2$ & $114^{* * *}$ & $22.94[19.93,25.96]$ \\
\hline & CC & $-6.7 \pm 0.1$ & $-55^{* * *}$ & $-6.55[-6.81,-6.29]$ & $-18.4 \pm 0.4$ & $-49 * * *$ & $-17.89[-18.67,-17.12]$ & $-12.8 \pm 0.3$ & $-48 * * *$ & $-12.44[-12.99,-11.89]$ \\
\hline & BC & $-0.5 \pm 0$ & $-13^{* * *}$ & $-0.6[-0.6,-0.5]$ & $-0.6 \pm 0.1$ & $-11 * * *$ & $-0.7[-0.8,-0.6]$ & $-0.6 \pm 0$ & $-11 * * *$ & $-0.6[-0.7,-0.5]$ \\
\hline & $\mathrm{CCxBC}$ & $0.3 \pm 0$ & $13^{* * *}$ & $0.35[0.3,0.4]$ & $1.2 \pm 0.1$ & $12^{* * *}$ & $1.27[1.08,1.46]$ & $0.8 \pm 0.1$ & $12 * * *$ & $0.78[0.66,0.9]$ \\
\hline & phyl var & - & - & $11.2[8.6,14.6]$ & - & - & $12.4[9.5,16.1]$ & - & - & $12.6[9.7,16.4]$ \\
\hline & res var & - & - & $0.42[0.39,0.46]$ & - & - & $0.52[0.48,0.57]$ & - & - & $0.52[0.48,0.57]$ \\
\hline \multirow{6}{*}{ forest shade } & intercept & $23.2 \pm 0.2$ & $116^{* * *}$ & $22.97[20.07,25.89]$ & $23.1 \pm 0.2$ & $110^{* * *}$ & $22.87[19.74,26.02]$ & $23 \pm 0.2$ & $109 * * *$ & $22.78[19.64,25.94]$ \\
\hline & $\mathrm{CC}$ & $-11.8 \pm 0.2$ & $-51 * * *$ & $-11.53[-12.01,-11.05]$ & $-36.7 \pm 0.8$ & $-45 * * *$ & $-35.71[-37.4,-34.05]$ & $-24.5 \pm 0.5$ & $-45 * * *$ & $-23.78[-24.9,-22.67]$ \\
\hline & BC & $-0.6 \pm 0$ & $-12 * * *$ & $-0.7[-0.8,-0.6]$ & $-0.8 \pm 0.1$ & $-10 * * *$ & $-0.9[-1.1,-0.8]$ & $-0.7 \pm 0.1$ & $-10 * * *$ & $-0.8[-1,-0.7]$ \\
\hline & $\mathrm{CCXBC}$ & $0.7 \pm 0.1$ & $12^{* * *}$ & $0.76[0.64,0.87]$ & $3.4 \pm 0.3$ & $12^{* * *}$ & $3.46[2.92,3.99]$ & $2 \pm 0.2$ & $12 * * *$ & $2.06[1.74,2.39]$ \\
\hline & phyl var & - & - & $11.8[9,15.3]$ & - & - & $13.7[10.5,17.7]$ & - & - & $13.8[10.5,17.9]$ \\
\hline & res var & - & - & $0.47[0.44,0.52]$ & - & - & $0.59[0.54,0.64]$ & - & - & $0.58[0.54,0.64]$ \\
\hline \multirow{6}{*}{ large gap } & intercept & $22.8 \pm 0.2$ & $111^{* * *}$ & $22.53[19.45,25.63]$ & $22.8 \pm 0.2$ & $108^{* * *}$ & $22.62[19.39,25.87]$ & $22.8 \pm 0.2$ & $108 * * *$ & $22.55[19.33,25.8]$ \\
\hline & CC & $-3.5 \pm 0.1$ & $-50 * * *$ & $-3.34[-3.48,-3.19]$ & $-7 \pm 0.2$ & $-44 * * *$ & $-6.78[-7.11,-6.45]$ & $-4.8 \pm 0.1$ & $-44 * * *$ & $-4.64[-4.86,-4.42]$ \\
\hline & BC & $-0.3 \pm 0$ & $-11 * * *$ & $-0.4[-0.4,-0.3]$ & $-0.2 \pm 0$ & $-10 * * *$ & $-0.3[-0.3,-0.2]$ & $-0.2 \pm 0$ & $-10 * * *$ & $-0.2[-0.3,-0.2]$ \\
\hline & $\mathrm{CCxBC}$ & $0.1 \pm 0$ & $13^{* * *}$ & $0.14[0.12,0.16]$ & $0.2 \pm 0$ & $12^{* * *}$ & $0.19[0.16,0.22]$ & $0.1 \pm 0$ & $12^{* * *}$ & $0.12[0.1,0.14]$ \\
\hline & phyl var & - & - & $13.3[10.2,17.3]$ & - & - & $14.5[11.1,18.8]$ & - & - & $14.5[11.1,18.8]$ \\
\hline & res var & - & - & $0.52[0.48,0.57]$ & - & - & $0.62[0.57,0.68]$ & - & - & $0.61[0.56,0.67]$ \\
\hline
\end{tabular}

Retained classic mixed model and Bayesian phylogenetically controlled mixed model for the relationships between the proportion of UV transmittance and biologically relevant descriptors of transparency. For all analyses, we took all 10 measurements per species. \# Proportion of UV transmittance was multiplied by 100 before analysis. We took species and wing within species as random factors in classic mixed models and species as random factor in Bayesian models. CC $=$ Colour contrast, $\mathrm{BC}=$ Brightness contrast. Bold values are statistically important factors associated with $95 \% \mathrm{Cl}$ excluding zero in Bayesian models, less important factors are associated with $90 \% \mathrm{Cl}$ excluding zero (with symbol $t)$ and $p$-values ( $\left.{ }^{*} p<0.05 ; * * p<0.01 ; * * * p<0.001\right)$ for the mixed model adjusted for type III sums of squares. Phy var and res var stand for phylogenetic variance and residual variance respectively. 
Table S13. Relationship between optical parameters of transparency and latitude.

\begin{tabular}{|c|c|c|c|c|}
\hline & \multirow[b]{2}{*}{ Factor } & \multicolumn{2}{|c|}{ Mixed model } & \multirow{2}{*}{$\begin{array}{c}\text { Bayesian } \\
\text { Estimate }[95 \% \mathrm{Cl}]\end{array}$} \\
\hline & & Estimate \pm se & t-value & \\
\hline \multirow{4}{*}{$\begin{array}{c}\text { Proportion of } \\
\text { UV } \\
\text { transmittance }\end{array}$} & intercept & $17.02 \pm 0.724$ & $23.51 * * *$ & $18.4[11,25.9]$ \\
\hline & Abs(Latitude) & $-0.04 \pm 0.042$ & -1.02 & $-0.07[-0.17,0.03]$ \\
\hline & Phylogenetic variance & - & - & $72.8[53.6,97.6]$ \\
\hline & Residual variance & - & - & $2.4[2.2,2.7]$ \\
\hline \multirow{4}{*}{$\begin{array}{c}\text { Mean } \\
\text { transmittance } \\
\text { over } \\
{[300-400] \mathrm{nm}}\end{array}$} & intercept & $39.66 \pm 3.01$ & $13.18 * * *$ & $22.1[0.1,67.9]$ \\
\hline & Abs(Latitude) & $-0.31 \pm 0.173$ & -1.76 & $-0.21[-0.73,0.0002]$ \\
\hline & Phylogenetic variance & - & - & $531.2[0,1363.3]$ \\
\hline & Residual variance & - & - & $24.1[0,52.4]$ \\
\hline \multirow{4}{*}{$\begin{array}{c}\text { Mean } \\
\text { transmittance } \\
\text { over } \\
{[400-700] \mathrm{nm}}\end{array}$} & intercept & $61.45 \pm 3.24$ & $18.96 * * *$ & $64.7[37.7,91.3]$ \\
\hline & Abs(Latitude) & $-0.38 \pm 0.187$ & $-2.02 *$ & $-0.5[-0.8,-0.1]$ \\
\hline & Phylogenetic variance & - & - & $936.8[684.7,1262.2]$ \\
\hline & Residual variance & - & - & $68.2[61.7,75.4]$ \\
\hline \multirow{4}{*}{$\begin{array}{c}\text { Mean } \\
\text { transmittance } \\
\text { over } \\
{[700-1100] \mathrm{nm}}\end{array}$} & intercept & $68.82 \pm 3.07$ & $22.41 * * *$ & $64.4[36.8,91.3]$ \\
\hline & Abs(Latitude) & $-0.29 \pm 0.17$ & -1.68 & $-0.5[-0.8,-0.1]$ \\
\hline & Phylogenetic variance & - & - & $936.6[683.1,1276.1]$ \\
\hline & Residual variance & - & - & $68.2[61.9,75.6]$ \\
\hline
\end{tabular}

Retained classic mixed model and Bayesian phylogenetically controlled mixed model for the relationship between optics, namely proportional UV transmittance, mean transmittance in the UV, in the human visible range, and in the near infrared range, and fixed factors, namely absolute value of latitude and wing length. For all analyses, we took all 10 measurements per species. We took species and wing within species as random factors in classic mixed models and species as random factor in Bayesian models. \# Proportion of UV transmittance was multiplied by 100 before analysis. Bold values are statistically important factors associated with $95 \% \mathrm{Cl}$ excluding zero in Bayesian models, less important factors are associated with $90 \% \mathrm{Cl}$ excluding zero (with symbol $t$ ) and $p$-values $\left({ }^{*} p<0.05 ;{ }^{* *} p<0.01 ;{ }^{* * *} p<0.001\right)$ for the mixed model adjusted for type III sums of squares. 
Figure S6. Correlation between mean transmittance in the near infrared 700-1100 nm range and mean transmittance in the UV range $300-400 \mathrm{~nm}(\mathrm{a})$, or the human-visible range $400-700 \mathrm{~nm}$ (b). Dashed lines indicate identical values.

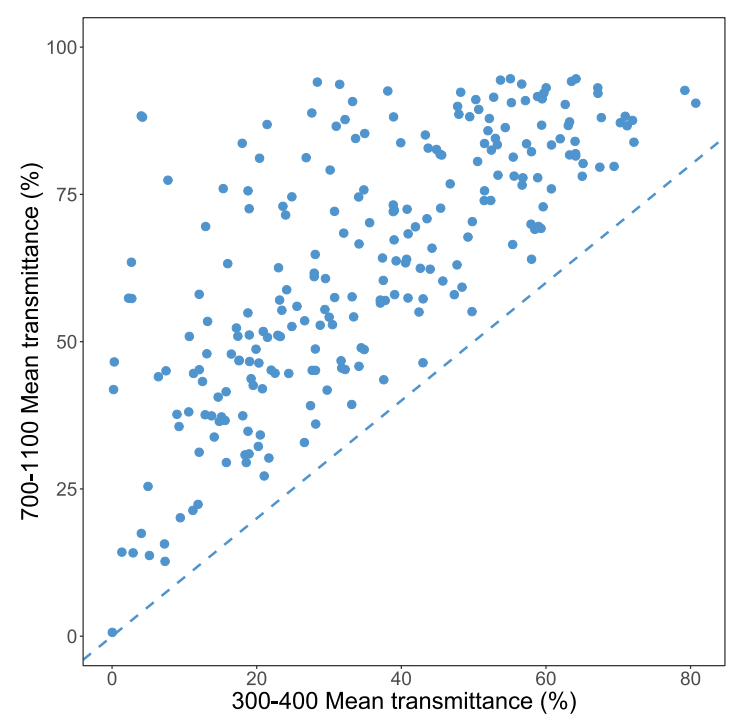

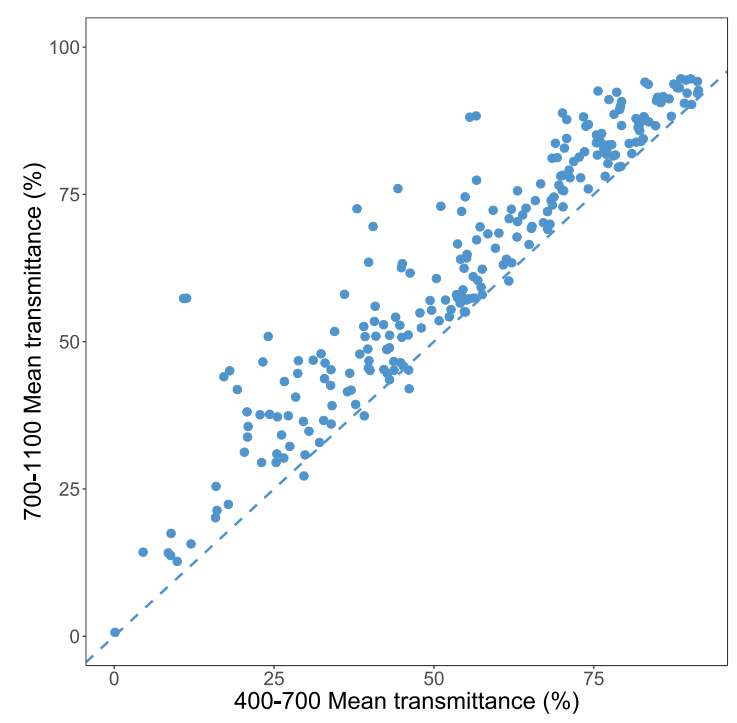




\section{REFERENCES}

Blomberg, S. P., T. Garland, and A. R. Ives. 2003. Testing for phylogenetic signal in comparative data: behavioral traits are more labile. Evolution 57:717-745.

Fritz, S. A., and A. Purvis. 2010. Selectivity in mammalian extinction risk and threat types: a new measure of phylogenetic signal strength in binary traits. Conservation Biology 24:1042-1051.

Pagel, M. 1999. Inferring the historical patterns of biological evolution. Nature 401:877-884. 